\title{
Neutrophil oxidative stress mediates obesity-associated vascular dysfunction and metastatic transmigration
}

\author{
Sheri A. C. McDowell1,2, Robin B. E. Luo ${ }^{1,3}$, Azadeh Arabzadeh1, Samuel Doré ${ }^{1,3}$, Nicolas C. Bennett ${ }^{1,2}$, \\ Valérie Breton', Elham Karimi ${ }^{1}$, Morteza Rezanejad ${ }^{4}$, Ryan R. Yang ${ }^{1,5}$, Katherine D. Lach ${ }^{6}$, \\ Marianne S. M. Issac ${ }^{6}{ }^{6}$, Bozena Samborska1, Lucas J. M. Perus ${ }^{1,2}$, Dan Moldoveanu1,7, Yuhong Wei, \\ Benoit Fiset ${ }^{1}$, Roni F. Rayes ${ }^{1,8}$, Ian R. Watson ${ }^{1,7}$, Lawrence Kazak ${ }^{1,7}$, Marie-Christine Guiot ${ }^{6,9}$, \\ Pierre O. Fiset ${ }^{6}$, Jonathan D. Spicer ${ }^{1,8,10}$, Andrew J. Dannenberg ${ }^{11}$, Logan A. Walsh ${ }^{1,3 凶}$ and \\ Daniela F. Quail ${ }^{1,2,8} \bowtie$
}

\begin{abstract}
Metastasis is the leading cause of cancer-related deaths, and obesity is associated with increased breast cancer (BC) metastasis. Preclinical studies have shown that obese adipose tissue induces lung neutrophilia associated with enhanced BC metastasis to this site. Here we show that obesity leads to neutrophil-dependent impairment of vascular integrity through loss of endothelial adhesions, enabling cancer cell extravasation into the lung. Mechanistically, neutrophil-produced reactive oxygen species in obese mice increase neutrophil extracellular DNA traps (NETs) and weaken endothelial junctions, facilitating the influx of tumor cells from the peripheral circulation. In vivo treatment with catalase, NET inhibitors or genetic deletion of Nos2 reversed this effect in preclinical models of obesity. Imaging mass cytometry of lung metastasis samples from patients with cancer revealed an enrichment in neutrophils with low catalase levels correlating with elevated body mass index. Our data provide insights into potentially targetable mechanisms that underlie the progression of BC in the obese population.
\end{abstract}

O besity is associated with increased cancer incidence ${ }^{1}$ and mortality ${ }^{2}$ from diverse tumor types including breast cancer (BC), and is estimated to be responsible for up to $20 \%$ of all cancer-related deaths ${ }^{2}$. With global prevalence rising ${ }^{3}$, obesity now competes with smoking tobacco as the leading preventable risk factor for cancer mortality ${ }^{4,5}$. In $\mathrm{BC}$, patient mortality is primarily caused by metastases within vital organs such as the lung, which are found in roughly $65 \%$ of patients with $\mathrm{BC}$ at autopsy ${ }^{6}$. In a retrospective analysis of 18,967 patients with early-stage BC, obesity at the time of diagnosis was associated with a $46 \%$ elevated risk of developing distant metastases within 10 years and a $38 \%$ elevated risk of BC mortality by 30 years, despite no relationship with locoregional recurrence ${ }^{7}$. A small retrospective analysis of 118 women who developed metastatic BC found that obesity may be associated with metastasis organ tropism, with earlier dissemination in obese women to lung or liver, but not brain or bone ${ }^{8}$. Understanding how obesity affects distinct metastatic microenvironments is imperative to develop therapeutic interventions.

A causal relationship between obesity-associated inflammation and $\mathrm{BC}$ metastasis was previously established, driven by alterations in the myeloid cell landscape in lung ${ }^{9,10}$. Specifically, obesity caused aberrant lung neutrophilia in association with enhanced spontaneous and experimental BC metastasis to this site in a neutrophil-dependent manner. Differences in experimental metastasis were observed as early as $48 \mathrm{~h}$ after intravenous (i.v.) transplantation of cancer cells, suggesting that changes in extravasation were likely at play. Given that the endothelial barrier acts as a gatekeeper for advanced stages of the metastatic cascade and the well-established impact of obesity on cardiovascular health, understanding the mechanistic relationship between obesity and cancer extravasation is a pertinent unanswered question. In the current study, we sought to investigate the role of obesity during BC extravasation to lung and identify how neutrophils become reprogrammed by obesity to facilitate this process.

\section{Results}

Obesity enhances BC extravasation by modifying the endothelium. To investigate the effects of obesity on cancer extravasation, we used a syngeneic BC cell line derived from the C57BL6 mouse mammary tumor virus-polyoma middle T antigen (MMTV-PyMT) model combined with a diet-induced obesity (DIO) model. Wild type (WT) C57BL6 female mice were enrolled on a low-fat (LF) $(10 \% \mathrm{kcal})$ or high-fat (HF) $(60 \% \mathrm{kcal})$ diet for 15 weeks (Fig. 1a,b) $)^{10}$, followed by tail vein injection of fluorescently labeled BC cells

'Rosalind and Morris Goodman Cancer Research Centre, McGill University, Montreal, Quebec, Canada. ${ }^{2}$ Department of Physiology, Faculty of Medicine, McGill University, Montreal, Quebec, Canada. ${ }^{3}$ Department of Human Genetics, McGill University, Montreal, Quebec, Canada. ${ }^{4}$ Departments of Psychology and Computer Science, University of Toronto, Toronto, Ontario, Canada. ${ }^{5}$ Department of Microbiology and Immunology, McGill University, Montreal, Quebec, Canada. ${ }^{6}$ Department of Pathology, Faculty of Medicine, McGill University, Montreal, Quebec, Canada. ${ }^{7}$ Department of Biochemistry, McGill University, Montreal, Quebec, Canada. ${ }^{8}$ Department of Medicine, Division of Experimental Medicine, McGill University, Montreal, Quebec, Canada. ${ }^{9}$ Montreal Neurological Institute, McGill University Health Centre, Montreal, Quebec, Canada. ${ }^{10}$ Department of Surgery, McGill University Health Centre, Montreal, Quebec, Canada. "1'Department of Medicine, Weill Cornell Medical College, New York, New York, USA. $\varpi_{e}$-mail: logan.walsh@mcgill.ca; daniela.quail@mcgill.ca 
(Fig. 1c). After $48 \mathrm{~h}$, histological analysis of lung tissues revealed a 2.3-fold increase in BC cells that had extravasated into the lung in obese versus lean mice (Fig. 1d-f). This was consistent with previous findings that obesity enhances experimental metastasis using bioluminescence ${ }^{10}$, and with new findings that obesity is associated with increased lung metastasis in a spontaneous C57BL6 MMTV-PyMT model (Extended Data Fig. 1a-c). To validate our observations, we performed a transwell transendothelial migration (TEM) assay, which revealed that serum from obese mice promoted TEM more efficiently than serum from lean mice (Fig. 1g,h and Extended Data Fig. 1d). These data support our hypothesis that obesity enhances BC cell extravasation.

We next uncoupled the relative contributions of diet versus adiposity to the extravasation phenotype. We used a genetically induced obesity (GIO) model comparing WT versus ob/ob mice on a standard rodent diet (SRD), in which $o b / o b$ mice gain weight from hyperphagia secondary to leptin deficiency (Extended Data Fig. 1e). BC cells were fluorescently labeled and injected via tail vein into GIO mice (Extended Data Fig. 1f). Similar to our findings in the DIO model, after $48 \mathrm{~h}$, fluorescence analysis of lung tissues revealed a 3.4-fold increase in $\mathrm{BC}$ cells that had extravasated into the lung in $o b / o b$ versus WT mice (Extended Data Fig. 1g-i). These data are consistent with previous findings that high adiposity causes obesity-associated neutrophilia and BC metastasis independent of $\operatorname{diet}^{10}$. Moreover, epidemiological studies have shown that loss of adiposity resulting from bariatric surgery is associated with a significant reduction in cancer-related mortality ${ }^{11}$.

Next, we determined whether obesity-associated BC extravasation was mediated through effects on BC cells or the endothelium (or both). We performed a modified TEM assay in which we pretreated either the BC cells (Fig. 1i,j) or endothelial cells (Fig. 1k,l) with serum from obese or lean mice before combining them in coculture. Pretreatment of BC cells with obese serum did not enhance TEM compared to lean serum (Fig. 1j). In fact, it had the opposite effect, suggesting that serum-derived factors from obese hosts actually make cancer cells less motile at the vascular interface. However, pretreatment of endothelial cells with obese serum significantly enhanced TEM compared to lean serum (Fig. 11), recapitulating the effects of LF/HF serum gradients (Fig. 1h). This suggests that obesity-enhanced extravasation occurs via modifications to the endothelium, rather than cancer cells.

Obesity increases vascular permeability by downregulating endothelial adhesions. We next explored how obesity changes the endothelium to facilitate metastatic extravasation. We performed RNA-sequencing (RNA-seq) on CD45-CD31+ lung endothelial cells from the DIO model to interrogate core transcription factor programs that underlie functional differences in response to obesity (Fig. 2a). Using DoRothEA, a gene set resource containing highly curated transcription factor-target interactions (regulons) $)^{12}$, we identified Foxo3 as the top upregulated transcriptional program in endothelial cells from obese versus lean mice (Fig. 2b,c). Studies have shown that FOXO3 regulates vascular remodeling and integrity ${ }^{13-15}$, therefore, we hypothesized that obesity may alter vascular permeability. LF or HF mice were injected i.v. with fluorescently conjugated dextran-Texas Red (to assess vascular permeability) and lectin-Dylight 488 (to assess vascular perfusion) (Fig. 2d). Obese mice exhibited enhanced vascular permeability in the lungs compared to lean mice, quantified by the amount of dextran-Texas Red that had leaked out of lectin-Dylight $488^{+}$blood vessels. This effect was independent of differences in vascular perfusion, as lectin-Dylight 488 was equal between groups (Fig. 2e-g). We confirmed this observation using two in vitro vascular permeability assays (Extended Data Fig. 2a,b). First, using a transendothelial electrical resistance assay (TEER), human microvascular endothelial cell (HMEC) monolayers exhibited reduced barrier integrity when treated with serum from obese versus lean mice (Fig. 2h). Second, using a dextran-FITC permeabilization assay, human umbilical vein endothelial cell (HUVEC) monolayers exhibited enhanced permeability when treated with serum from obese versus lean mice (Fig. 2i). These data indicate that obesity may enhance cancer extravasation by increasing vascular permeability.

In response to pathogen exposure, one function of innate immune cells is to stimulate vascular inflammation by altering the expression of adhesion proteins along apical sides and intercellular junctions of endothelial cells to facilitate immune infiltration ${ }^{16,17}$. Therefore, we hypothesized that obesity may alter endothelial adhesions, mimicking a physiological response to infection. We performed western blot analysis for NG2 (pericyte marker), CD31 (endothelial marker) and ten proteins related to vascular adhesions, using whole-lung tissues from LF and HF mice (Fig. 2j). Expression of the tight junction protein, junctional adhesion molecule-1 (JAM1), was reduced by $59 \%$ in lung tissues from obese versus lean mice (Fig. 2k). We next quantified the expression of vascular adhesion proteins in a cell-specific manner using immunofluorescent costaining with CD31. We confirmed that JAM1 was significantly reduced by $57 \%$ in the lung endothelium of obese versus lean mice (Fig. 21 and Extended Data Fig. 2c,d). Finally, we fractionated HUVECs treated with serum from obese versus lean mice, and confirmed reduced expression of JAM1 on the plasma membrane in response to obesity (Fig. $2 \mathrm{~m}$ ). We did not see a concurrent increase in cytoplasmic JAM1, suggesting that obesity alters overall JAM1 levels rather than subcellular localization. As a control, we functionally confirmed that JAM1 knockdown in endothelial cells was sufficient on its own to enhance vascular permeability via TEER and TEM assays (Fig. 2n,o and Extended Data Fig. 2e-h).

Fig. 1 | Obesity enhances BC extravasation by modifying the endothelium. $\mathbf{a}$, Weight curves for the DIO model. $n=10$ mice per condition; mean \pm s.e.m. $\mathbf{b}$, DIO model average weight after 15 weeks of LF or HF diet. $n=10$ mice per condition; mean \pm s.e.m.; two-tailed Mann-Whitney test. c, Schematic illustration of the in vivo extravasation assay corresponding to $\mathbf{d}-\mathbf{f}$. $\mathbf{d}$, Immunofluorescence of lung vascular density in the in vivo extravasation assay (percentage of $\mathrm{CD} 1^{+}$cells of total DAPI+ cells). LF, $n=7$ mice; HF, $n=9$ mice; mean \pm s.e.m.; two-tailed Student's $t$-test. e, Fluorescence quantification of Py230 cancer cell extravasation in lung tissue. $L F, n=8$ mice; $H F, n=9$ mice; mean \pm s.e.m.; two-tailed Mann-Whitney test. f, Representative immunofluorescence image for trial shown in c-f. Scale bars, $100 \mu \mathrm{m}$. $\mathbf{g}$, Schematic illustration of the in vitro TEM assay. $\mathbf{h}$, Quantification of TEM as depicted in $\mathbf{g}$ : $n=4$ transwells per condition in all cases; transwells represent individual experimental replicates with similar results using serum from different mice; mean \pm s.e.m.; two-tailed Student's $t$-test. $\mathbf{i}$, Schematic illustration of a modified in vitro TEM assay, in which BC cells were pretreated with serum from LF or HF mice, and a 0-2\% FBS gradient was used. j, Quantification of modified in vitro TEM as in i. Py230, $n=4$ transwells per condition, two-tailed Mann-Whitney test; E0771, $n=6$ transwells per condition, two-tailed Student's $t$-test; MDA-MB-231, $n=4$ transwells per condition. Transwells represent individual experimental replicates with similar results using serum from different mice; two-tailed Student's $t$-test. All graphs show mean \pm s.e.m. $\mathbf{k}$, Schematic illustration of a modified in vitro TEM assay, in which endothelial cells are pretreated with serum from LF or HF mice, and a 0-2\% FBS gradient is used. l, Quantification of modified TEM as depicted in k. Py230, $n=8$ transwells per condition, two-tailed Student's $t$-test; E0771, $n=4$ transwells per condition, two-tailed Student's $t$-test; MDA-MB-231, $n=4$ transwells per condition; transwells represent individual experimental replicates with similar results using serum from different mice; two-tailed Student's $t$-test. All graphs show mean \pm s.e.m. NS, not significant. 
Endothelial barrier integrity is regulated by neutrophils during obesity. It was previously shown that obesity-associated BC metastasis to lung is dependent on neutrophils ${ }^{10}$. Therefore, we evaluated the contribution of neutrophils to vascular permeability. We depleted neutrophils in obese mice with an antibody against Gr1 (RB6-8C5) $)^{10,18}$ versus IgG followed by i.v. administration

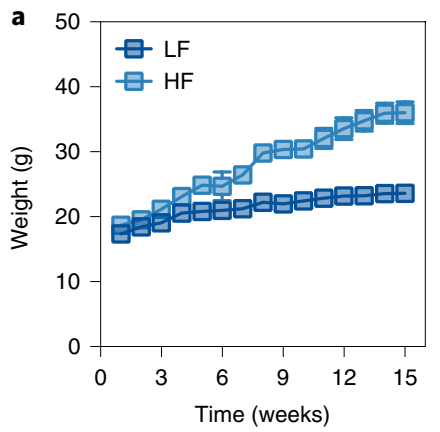

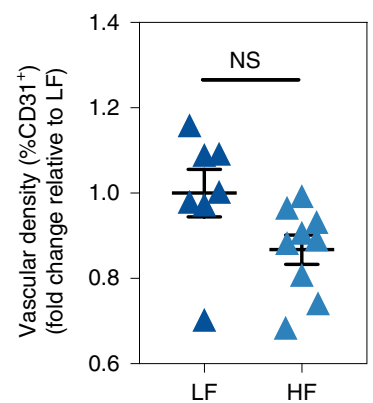

g

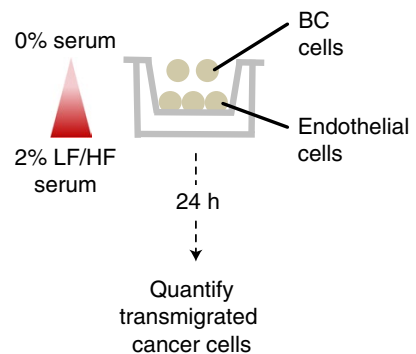

cancer cells

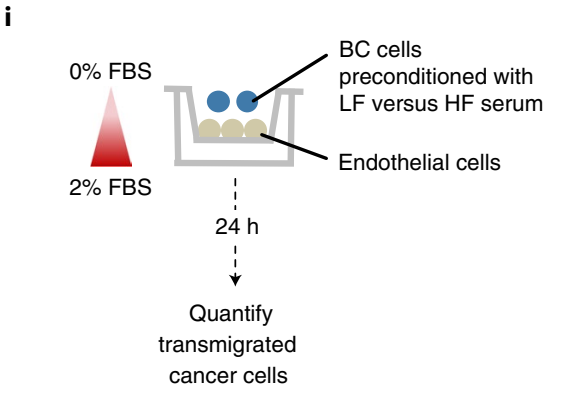

k

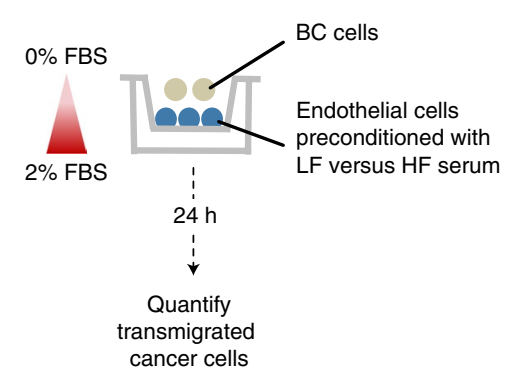

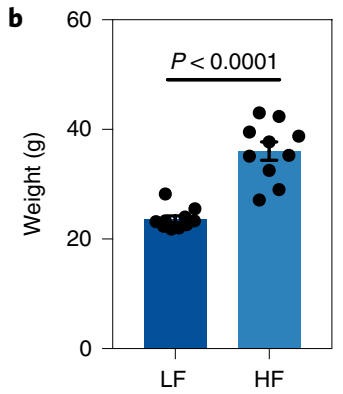
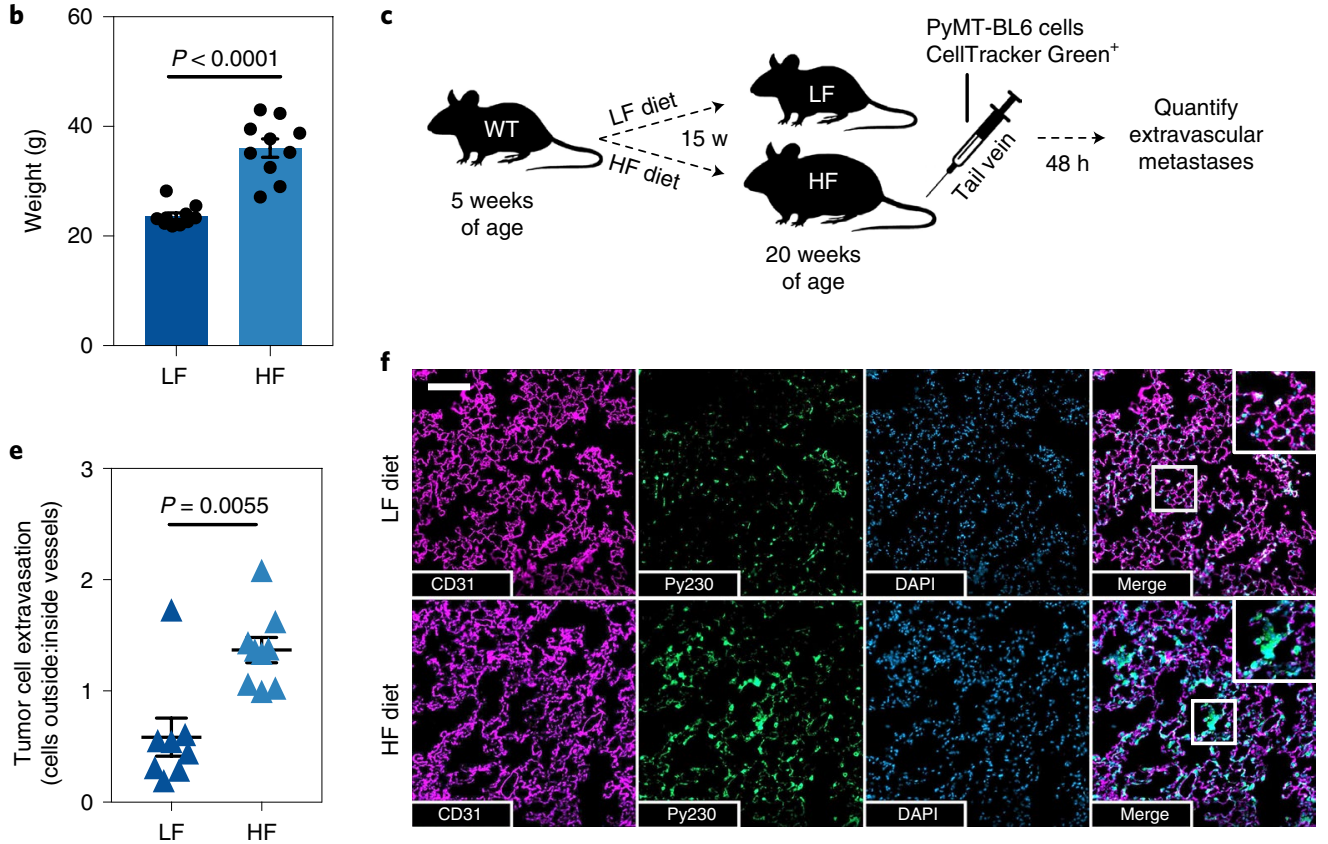
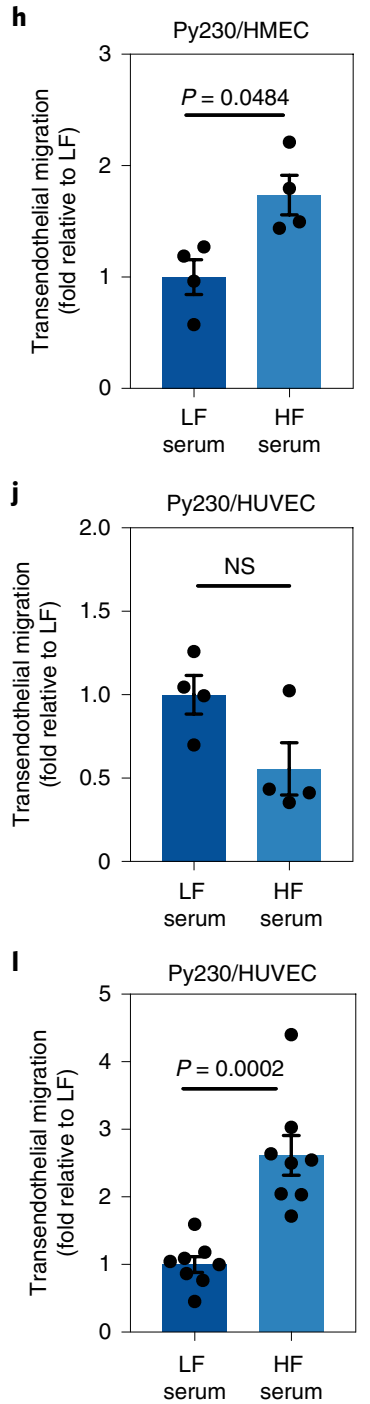
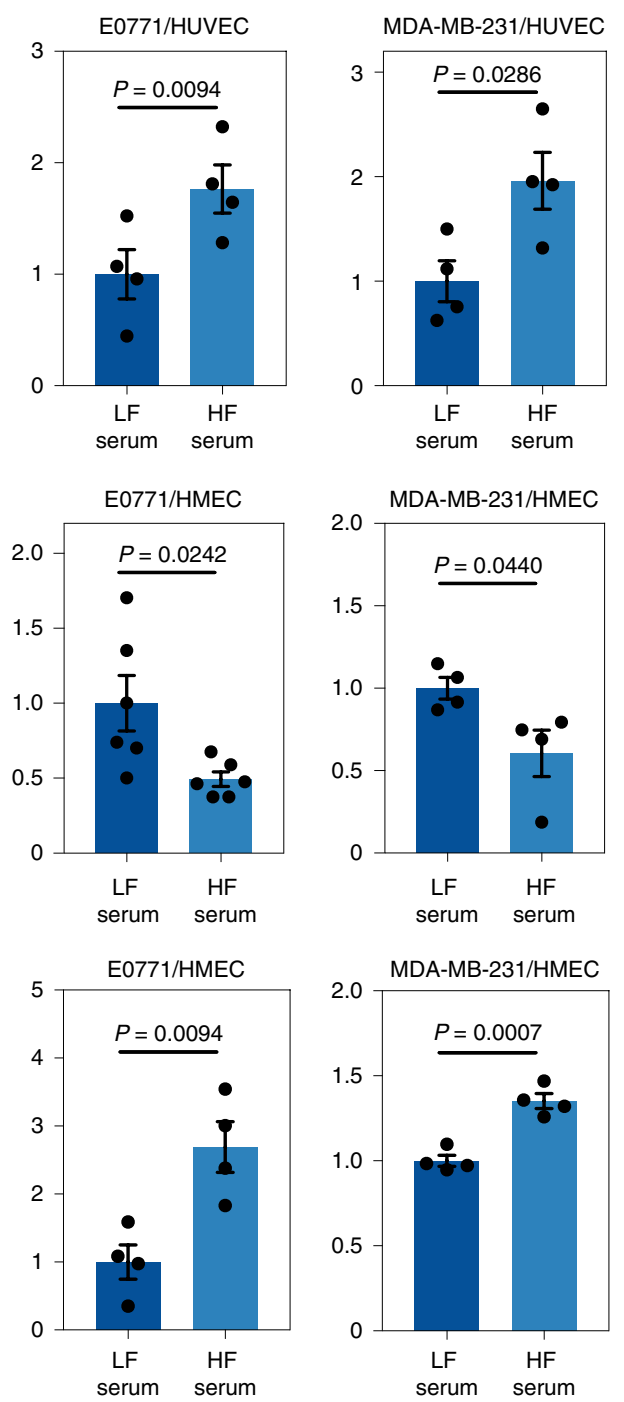
of dextran-Texas Red and lectin-Dylight488 (Fig. 3a). Obese/ anti-Gr1 mice exhibited reduced lung vascular permeability compared to obese/IgG mice (Fig. 3b-d), coinciding with increased JAM1 expression within the endothelium (Fig. 3e). We performed dextran-FITC and TEM in vitro assays to validate our findings, using endothelial cells pretreated with conditioned media from LF or HF bone-marrow-derived neutrophils. We found increased HUVEC monolayer permeability (Fig. 3f) and BC TEM (Fig. $3 g$ and Extended Data Fig. 3a,b) in response to neutrophil-conditioned media (nCM) from obese versus lean mice, recapitulating the effects of serum (Figs. 1h,l and 2h,i). Together, these data indicate that neutrophils from lean and obese mice have functionally distinct consequences on the endothelium.

Given the similarities between our in vitro findings using nCM (Fig. 3f,g) versus serum (Figs. 1h,l and 2h,i), we hypothesized that serum effects are partially due to factors derived from neutrophils within the host. To test this, we depleted neutrophils from the DIO model using a combination of anti-Ly6G (1A8) with a mouse IgG2a antibody against rat kappa light chain (MAR 18.5, binds the anti-Ly6G antibody; Extended Data Fig. 3c,d). This method is more specific against mature neutrophils compared to anti-Gr1, and effectively depletes neutrophils in C57BL6 mice unlike anti-Ly6G alone ${ }^{18}$. We isolated serum from these mice, and found HMEC barrier integrity (TEER) was reduced in response to serum from $\mathrm{HF} / \mathrm{IgG}$ versus LF/IgG hosts (Fig. 3 h), which improved when HF mice were depleted of neutrophils (Fig. 3 h). This suggests that the in vitro effects of serum are dependent in part on the presence of neutrophils in the host at the time of serum isolation. Moreover, when mice were injected with BC cells via tail vein, neutrophil depletion reduced $\mathrm{BC}$ extravasation in the lung in obese mice but not lean mice (Fig. 3i), indicating that the effects of neutrophils on $\mathrm{BC}$ extravasation are specific to obesity, in agreement with previous findings ${ }^{10}$.

Obesity reprograms neutrophils to increase reactive oxygen species (ROS) production. To interrogate putative differences between neutrophils from obese and lean hosts, we performed RNA-seq on FACS-purified lung neutrophils $\left(\mathrm{CD} 45^{+} \mathrm{CD} 11 \mathrm{~b}^{+} \mathrm{Ly} 6 \mathrm{G}^{\mathrm{hi}} \mathrm{Ly} 6 \mathrm{C}^{\mathrm{lo}}\right)$ from the DIO model (Fig. $4 \mathrm{a}$ and Extended Data Fig. 4a,b). Gene Set Enrichment Analysis (GSEA) and Ingenuity Pathway Analysis (IPA) revealed that genes related to ROS were enriched in neutrophils from obese versus lean mice (Fig. $4 b, c)$. We also found a downregulation of genes encoding proteins involved in antioxidant activity (including catalase and several peroxiredoxins, which are highly catalytic toward $\mathrm{H}_{2} \mathrm{O}_{2}$ ) and an upregulation of genes encoding proteins involved in the generation of free radicals (including inducible nitric oxide synthase and components of nicotinamide adenine dinucleotide phosphate oxidase) in neutrophils from obese versus lean mice (Fig. 4d). To validate these findings, we used a fluorogenic probe (CellROX) that is oxidized by ROS within live cells and can be quantified by flow cytometry. In tumor-bearing mice, we found elevated CellROX ${ }^{+}$neutrophils in lungs from obese versus lean mice (Fig. 4e and Extended Data Fig. 4c,d). This was specific to neutrophils, as no differences in CellROX were observed for other innate cells including monocytes or macrophages (Extended Data Fig. $4 \mathrm{e}-\mathrm{h}$ ). Together, these findings were reminiscent of the role of neutrophil-derived ROS in microvascular injury and leakage during acute inflammation ${ }^{19}$.

Neutrophils rely on free radicals for innate immune defenses (Fig. 4f). Neutrophil oxidative burst is predominantly regulated by myeloperoxidase (MPO) that requires $\mathrm{H}_{2} \mathrm{O}_{2}$ to generate hypochlorous acid $(\mathrm{HOCl})$, a potent oxidant and proinflammatory molecule. To offset this process, $\mathrm{H}_{2} \mathrm{O}_{2}$ is converted to $\mathrm{H}_{2} \mathrm{O}$ and $\mathrm{O}_{2}$ by glutathione peroxidase or, more potently, catalase-one of the most efficient enzymes with the highest turnover rate known. Using colorimetric assays, we found decreased catalase activity with no change in superoxide dismutase (Fig. $4 \mathrm{~g}$ and Extended Data Fig. 4i,j), and increased MPO and hypochlorite (proxy for $\mathrm{HOCl}$ ) in neutrophils from obese versus lean mice (Fig. $4 \mathrm{~h}, \mathrm{i}$ and Extended Data Fig. 4k). This was driven by obesity rather than the tumor itself, as hypochlorite was elevated to the same degree in both tumor-bearing and nontumor-bearing settings (Fig. 4h and Extended Data Fig. 4l). In accordance with RNA-seq (Fig. 4d), we also found increased iNOS activity in neutrophils in response to obesity (Fig. 4j) - an enzyme required for production of nitric oxide and peroxynitrite, which primes neutrophils for enhanced oxidative burst $\mathrm{t}^{20,21}$. This was observed in concordance with an increase in serum nitrotyrosine, which is indicative of peroxynitrite-mediated tyrosine nitration (Fig. 4k).

Neutrophil-ROS modulates vascular integrity and enhances BC extravasation during obesity in a reversible manner. We next tested whether obesity-associated ROS regulates vascular integrity and $\mathrm{BC}$ metastasis in vivo. BC cells were fluorescently labeled and

Fig. 2 | Obesity increases vascular permeability by downregulating endothelial adhesions. a, Volcano plot showing differentially expressed genes from RNA-seq of lung endothelial cells isolated from HF versus LF mice. $\mathbf{b}$, Gene regulatory network diagram highlighting the top differentially active transcriptional regulator (Foxo3) in lung endothelial cells from HF versus LF mice. Oval depicts genes under the regulation of transcription factors (TFs, rectangle). Transcription factor color indicates the direction of activity in HF versus LF (red, increased activity and blue, decreased activity). c, Volcano plot of top differentially active master regulators in HF versus LF lung endothelial cells. $\mathbf{d}$, Schematic illustration of the in vivo vascular permeability assay corresponding to e-g. e, In vivo vascular permeability analysis in lung. LF, $n=6$ mice; HF, $n=7$ mice; mean \pm s.e.m.; two-tailed Student's $t$-test. f, In vivo vascular perfusion analysis in lung. LF, $n=6$ mice; $H F, n=7$ mice; mean \pm s.e.m.; two-tailed Student's $t$-test. g, Representative immunofluorescence image for data shown in e. Scale bar, $500 \mu \mathrm{m}$. h, HMEC monolayer permeability via TEER. Dotted line indicates treatment with serum from LF or HF mice. $n=4$ transwells per condition representing individual experimental replicates with similar results using serum from different mice; mean \pm s.e.m.; two-tailed Student's t-test. i, In vitro dextran-FITC permeability assay depicting HUVEC monolayer permeability following treatment with serum from LF or HF mice. $n=6$ transwells per condition representing individual experimental replicates with similar results using serum from different mice; mean \pm s.e.m.; two-tailed Student's $t$-test. j, Western blot analysis for vascular adhesion proteins on lung tissues isolated from LF or HF mice ( $n=4$ mice per group). One representative $\beta$-Actin band is shown. $\mathbf{k}$, Quantification of JAM1 protein from western blot analysis in $\mathbf{j}$. $n=4$ mice per condition; mean \pm s.e.m.; two-tailed Student's t-test. $\beta$-actin was used as a loading control for normalization. I, Immunofluorescence of CD31 colocalization with vascular adhesion proteins in lung tissues from HF relative to LF mice. JAM1, $n=7$ mice; $N$-cadherin, $n=4$ mice; all others, $n=5$ mice; mean \pm s.e.m.; two-tailed Student's $t$-test. $\mathbf{m}$, Representative western blot of cytoplasm- and membrane-fractionated HUVEC endothelial cells treated with serum from LF or HF mice. Repeated three times with similar results. $\mathbf{n}$, TEER from HMEC monolayers genetically modified to express a JAM1 shRNA (shJAM1) or a scramble control shRNA (shSCR). $n=5$ transwells per condition representing individual experimental replicates with similar results; mean \pm s.e.m.; two-tailed Student's $t$-test. o, Py230 TEM across shSCR or shJAM1 HMEC monolayers. Two independent JAM1 shRNAs were used. Graphs show $n=8$ transwells for shJAM1 $n=4$ transwells for shJAM12 and $n=12$ transwells for shSCR, representing $n=8$ experimental replicates matched to shJAM ${ }^{1}$ and $n=4$ matched to shJAM ${ }^{2}$ that were used for statistics and had similar results; mean \pm s.e.m.; two-tailed Student's $t$-test for the indicated comparisons. Western blots in $\mathbf{j}$ and $\mathbf{m}$ are cropped and uncropped blot images are shown in the source data. 
injected via tail vein into LF or HF mice treated with catalase conjugated to polyethylene glycol (PEG) (Fig. 5a), which prolongs the activity of catalase in vivo ${ }^{22}$ and effectively reduces serum hypochlorite (Fig. 5b). After $48 \mathrm{~h}$, fluorescence analysis of lung tissues revealed reduced $\mathrm{BC}$ extravasation in obese mice treated with catalase versus vehicle (Fig. $5 c$ and Extended Data Fig. 5a). This effect was not observed when mice were treated with vitamin $\mathrm{E}$ (Fig. 5d and Extended Data Fig. 5b), which is inefficient at scavenging $\mathrm{HOCl}^{23}$ (Fig. 5e). Using immunofluorescence costaining, we also observed a higher frequency of $\mathrm{JAM} 1^{+} \mathrm{CD} 31^{+}$vessels in obese mice treated with catalase versus vehicle (Fig. $5 \mathrm{f}$ and Extended Data Fig. 5c). Catalase treatment had a similar effect in the GIO model, whereby $o b / o b$ mice exhibited reduced BC extravasation following treatment with catalase versus vehicle (Fig. $5 \mathrm{~g}$ and Extended Data Fig. 5d,e), concomitant with elevated JAM1 ${ }^{+} \mathrm{CD} 31^{+}$vessels (Fig. 5h). We observed no effect of catalase in lean mice from the DIO model (LF diet, Fig. 5c,f) or GIO model (WT, normal diet; Fig. $5 \mathrm{~g}, \mathrm{~h}$ ). These data support the notion that obesity-associated ROS regulates vascular integrity and $\mathrm{BC}$ extravasation to lung.

To test the role of neutrophil-ROS on BC extravasation, we supplemented nCM with catalase in cell-based assays. Pretreatment of endothelial cells with $\mathrm{nCM}$ from HF versus LF mice enhanced BC TEM and endothelial permeability coinciding with $48 \%$ reduced JAM1 expression, and this was reversed with catalase (Fig. 5i-k). This was unlikely due to a direct effect of catalase within endothelial cells, as Cat-short-hairpin RNA (shRNA) in HMECs had the a

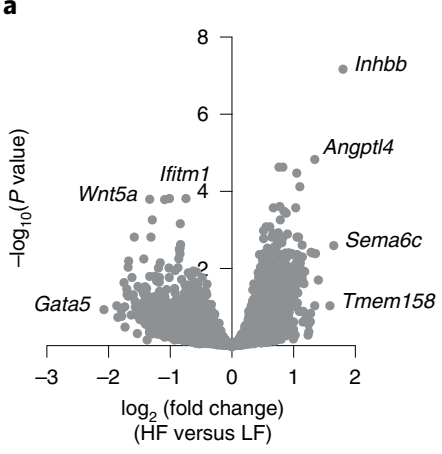

b

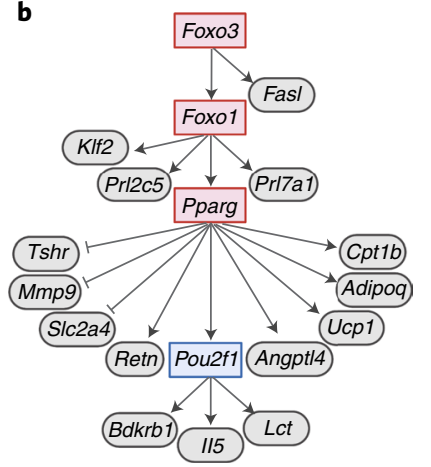

c

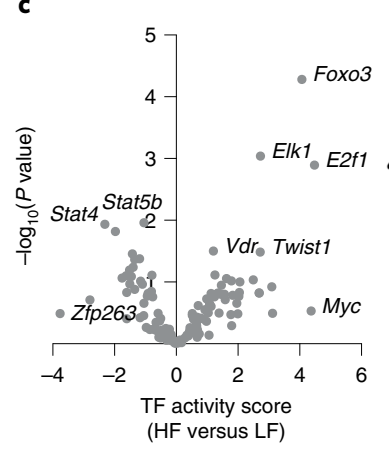

d

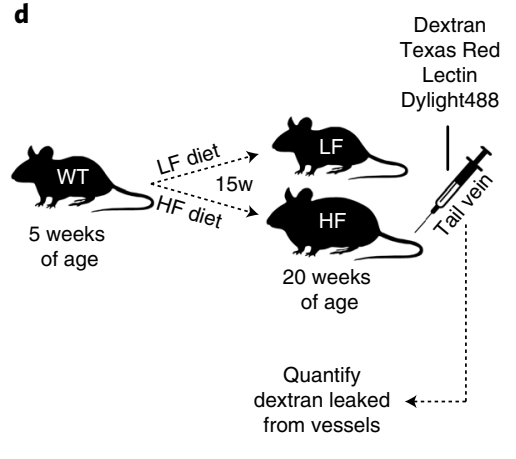

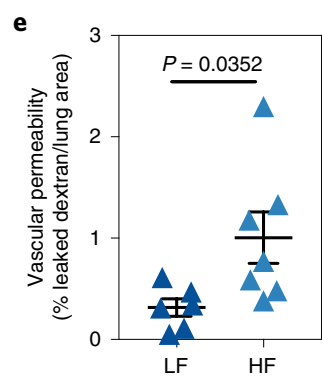
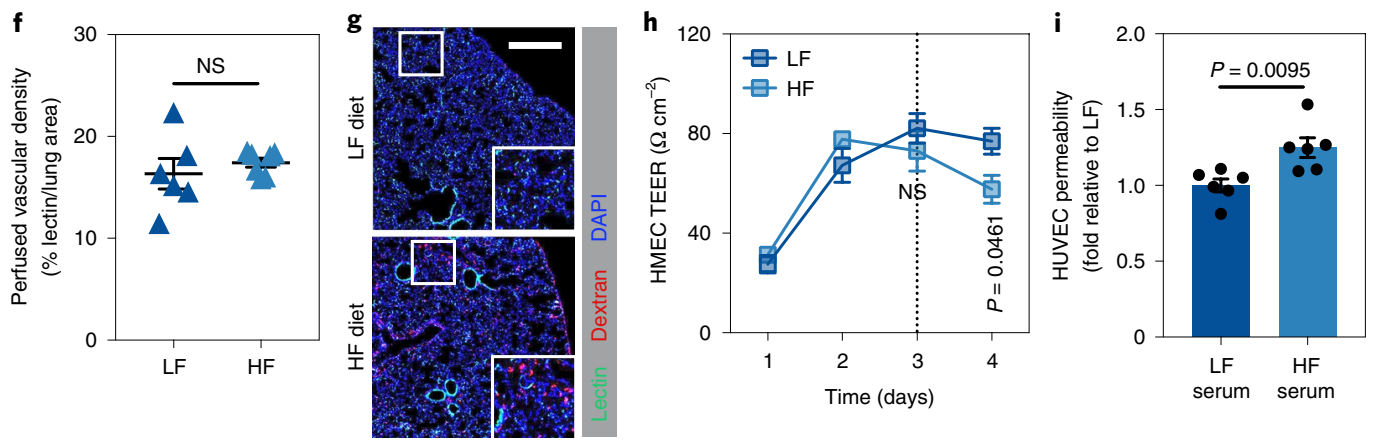

j
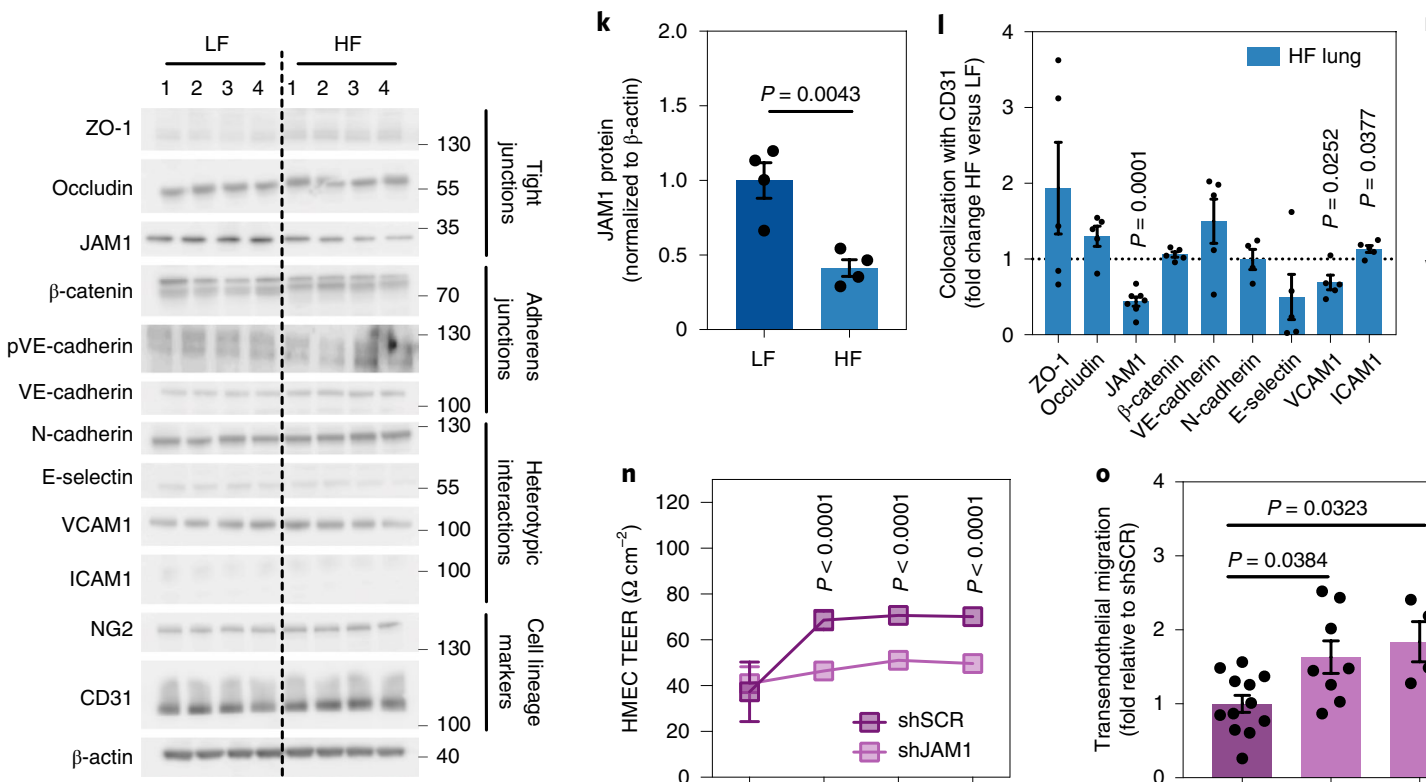

m
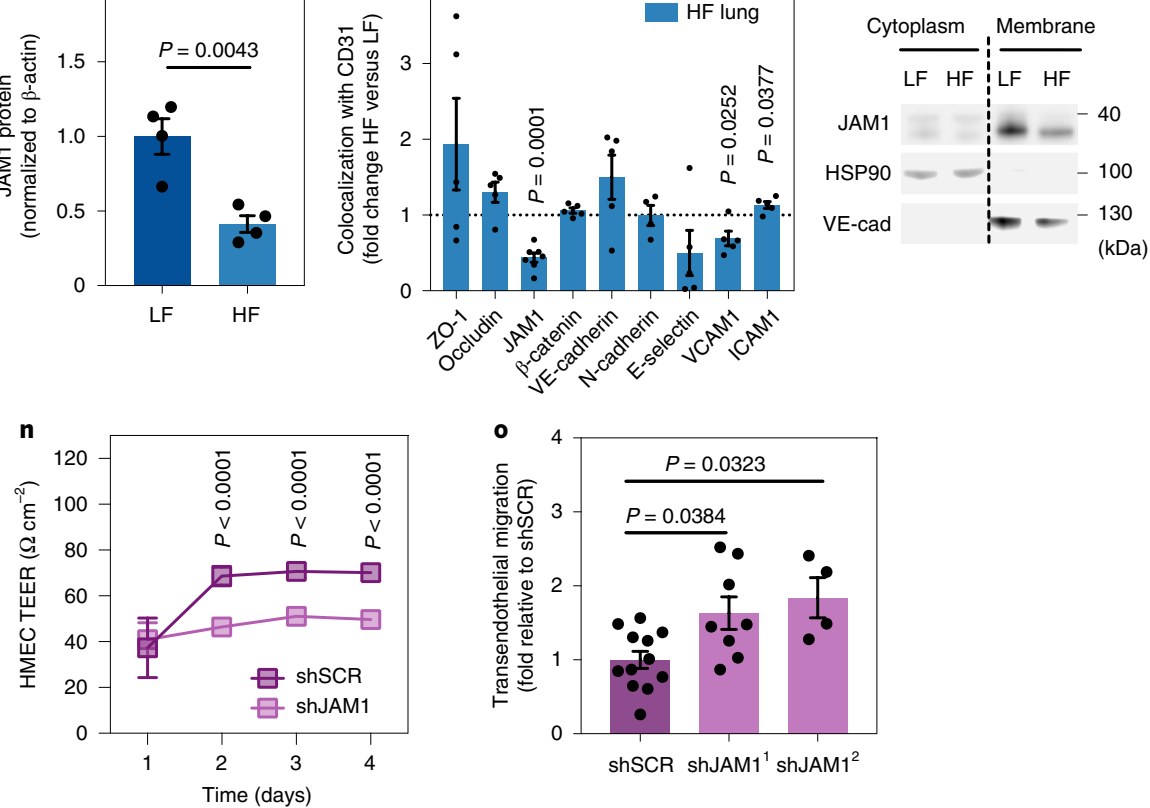
a
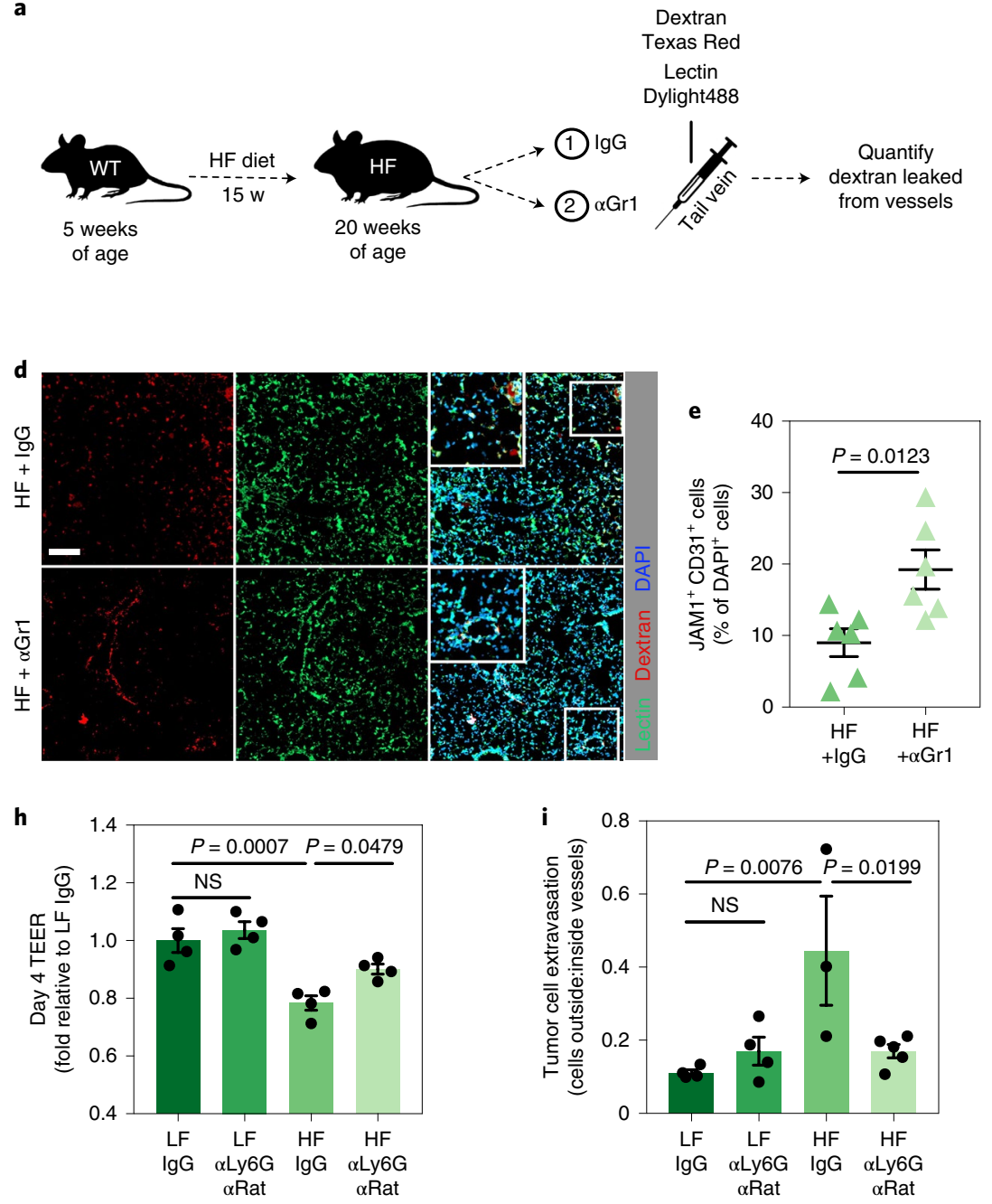
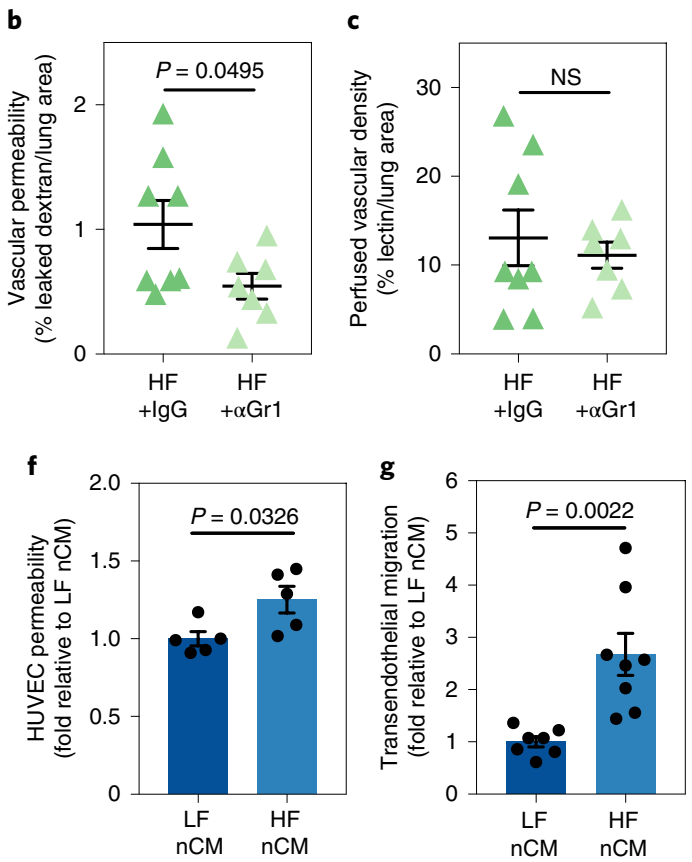

Fig. 3 | Endothelial barrier integrity is regulated by neutrophils during obesity. a, Schematic of trial design for in vivo vascular permeability assay with neutrophil depletion via $\alpha \mathrm{Gr} 1$ in HF mice, corresponding to b-e. b, In vivo vascular permeability analysis in lung from HF mice treated with $\alpha \mathrm{Gr} 1$ or IgG. $\mathrm{HF}+\mathrm{lgG}, n=8$ mice; HF $+\alpha \mathrm{Gr} 1, n=7$ mice; mean \pm s.e.m.; two-tailed Student's $t$-test. $\mathbf{c}$, In vivo vascular perfusion analysis in lung from HF mice treated with $\alpha \mathrm{Gr} 1$ or lgG. HF $+\operatorname{lgG}, n=8$ mice; HF $+\alpha \mathrm{Gr} 1, n=7$ mice; mean \pm s.e.m.; two-tailed Student's $t$-test. $\mathbf{d}$, Representative immunofluorescence image for a-c. Scale bar, $100 \mu \mathrm{m}$. e, Immunofluorescence analysis of JAM1 expression in CD31+ cells in lung from HF mice treated with $\alpha \mathrm{Gr} 1$ or IgG. $n=6$ mice per condition; mean \pm s.e.m.; two-tailed Student's $t$-test. f, In vitro dextran-FITC permeability assay depicting HUVEC monolayer permeability following treatment with bone-marrow $\mathrm{nCM}$ from LF or HF mice. $n=5$ transwells per condition representing experimental replicates using $\mathrm{nCM}$ from individual mice; mean \pm s.e.m.; two-tailed Student's $t$-test. g, Py230 TEM across HUVEC monolayers treated with bone-marrow nCM from LF or HF mice. LF nCM, $n=7$ transwells with $\mathrm{nCM}$ from seven mice; HF nCM, $n=8$ transwells with $\mathrm{nCM}$ from eight mice; mean \pm s.e.m.; two-tailed Student's $t$-test. $\mathbf{h}$, TEER from HMEC monolayers treated with serum collected from LF or HF mice $\pm \alpha$ Ly6G/ $\alpha$ Rat (neutrophil depletion) or lgG control. $n=4$ transwells per condition representing experimental replicates using serum from individual mice; mean \pm s.e.m.; one-way ANOVA and Bonferroni multiple comparisons test. i, Fluorescence quantification of Py230 cancer cell extravasation in lung tissue from LF or HF mice $\pm \alpha L y 6 G / \alpha R a t$ (neutrophil depletion) or lgG control. LF IgG, $n=4$ mice; LF $\alpha$ Ly6G/ $\alpha$ Rat, $n=4$ mice; HF IgG, $n=3$ mice, HF $\alpha$ Ly6G/ $\alpha$ Rat, $n=5$ mice; mean \pm s.e.m.; one-way ANOVA and Bonferroni multiple comparisons test.

opposite effect (Extended Data Fig. 5f). Catalase was ineffective when added to nCM from lean hosts (Fig. 5i,j) or when added after conditioned media collection (compared with during, Fig. 5l). This observation was phenocopied when nCM was supplemented with methionine, a potent $\mathrm{HOCl}$ scavenger (Fig. 51). These findings suggest that catalase influences the composition of factors released into conditioned media by obesity-derived neutrophils.

Obesity alters the secretory profile of neutrophils and enhances NETosis. To identify factors differentially released by neutrophils in obese versus lean hosts, we performed a cytokine array detecting 111 soluble proteins in lung $\mathrm{nCM}$ from the DIO model. MMP9 and Lipocalin-2 (LCN2) were the top upregulated proteins secreted by neutrophils from obese versus lean mice (Fig. 6a and Extended Data Fig. 6a,b), which are components of neutrophil tertiary and secondary granules, respectively, and regulate vascular dysfunction in mouse models of diabetes and atherosclerosis ${ }^{24,25}$. This was verified at the transcriptional level, as $M m p 9$ was 800 -fold higher in neutrophil RNA-seq from HF versus LF mice $\left(P<1 \times 10^{-300}\right.$, Fig. 6b,c), and at the functional level, where addition of an MMP9 inhibitor to nCM from obese mice reduced BC TEM (Fig. 6d). Neutrophil RNA-seq also verified elevated Lcn2 and Ltf (lactoferrin) in HF versus LF hosts (Fig. 6b), which both encode proteins that supply host cells with fuel for ROS generation due to their 
a

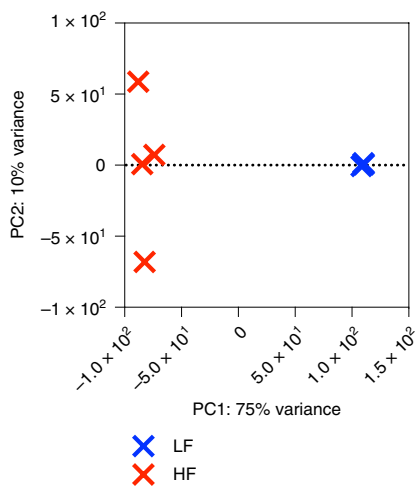

b

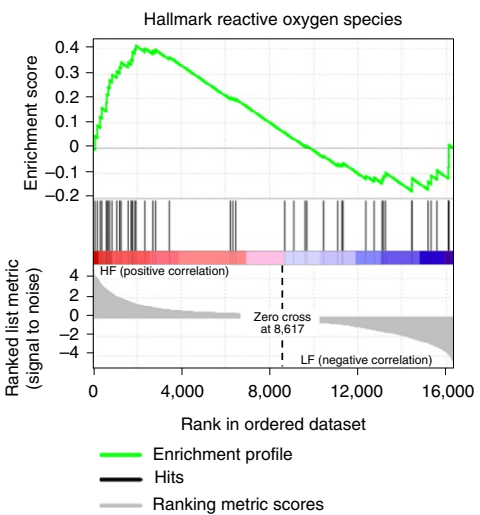

c

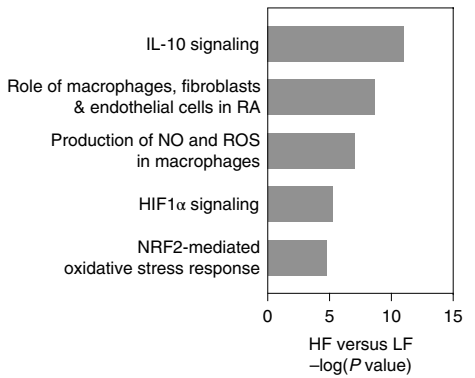

d

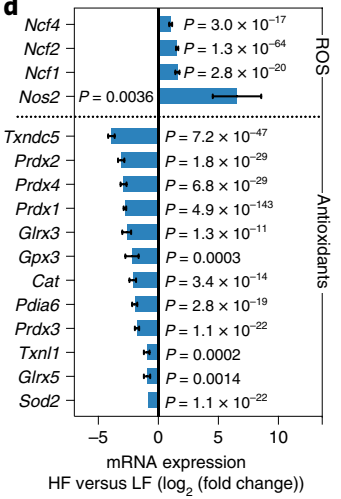

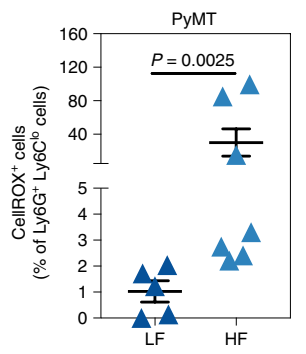

h

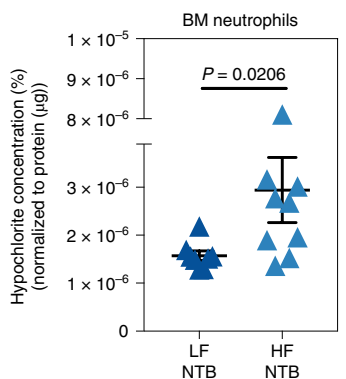

f
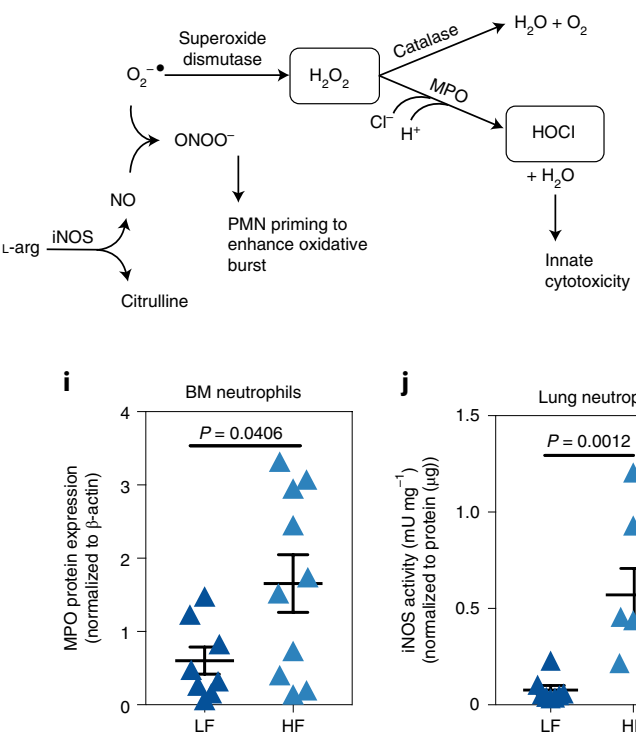

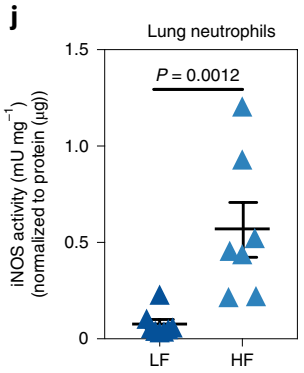

g
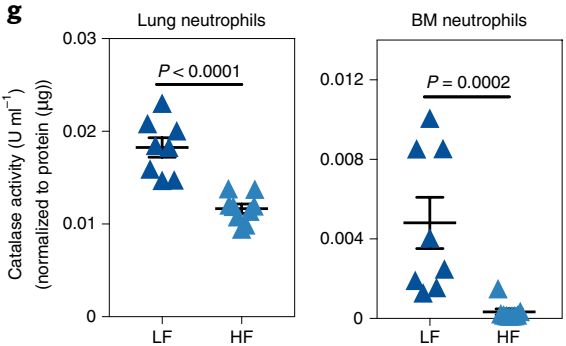

Fig. 4 | Obesity reprograms neutrophils to increase ROS production. a, Principal component analysis of RNA-seq of lung neutrophils isolated from LF ( $n=3$ mice) or HF ( $n=4$ mice) mice. b, GSEA showing that hallmark ROS are a top enriched pathway from neutrophil RNA-seq (NES $=1.9$, FDR $q=0.038)$. c, Curated list of significantly changing pathways predicted by IPA from neutrophil RNA-seq. d, RNA-seq differentially expressed genes in HF ( $n=4$ mice) relative to LF ( $n=3$ mice) lung neutrophils, showing genes relevant to oxidative stress. Data are displayed as log $f_{2}$ fold $H F$ versus $L F$ with standard error. e, In vivo CellROX flow cytometry assay, showing the percentage of CellROX+ neutrophils in lungs from LF or HF PyMT tumor-bearing mice. LF, $n=5$ mice; HF, $n=7$ mice; mean \pm s.e.m.; two-tailed Mann-Whitney test. f, Schematic of neutrophil oxidative burst, outlining key molecular players in obesity. g, Catalase activity assay using lung neutrophils (left, two-tailed Student's t-test) or bone-marrow neutrophils (right, two-tailed Mann-Whitney test) isolated from LF or HF mice. LF, $n=8$ mice; HF, $n=9$ mice; mean \pm s.e.m. $\mathbf{h}$, Hypochlorite concentration in bone-marrow neutrophils isolated from LF or $\mathrm{HF}$ nontumor-bearing (NTB) mice. LF, $n=8$ mice; HF, $n=9$ mice; mean \pm s.e.m.; two-tailed Mann-Whitney test. i, MPO protein levels by western blot in bone-marrow neutrophils isolated from LF or HF mice. LF, $n=8$ mice; HF, $n=10$ mice; mean \pm s.e.m.; two-tailed Student's $t$-test. $\beta$-actin was used as a loading control for normalization. Uncropped blot images for all replicates are shown in the source data. $\mathbf{j}$, iNOS activity assay using lung neutrophils (left, two-tailed Mann-Whitney test; LF, $n=8$ mice; HF, $n=7$ mice) or bone marrow (right, two-tailed Student's $t$-test; LF, $n=8$ mice; HF, $n=9$ mice) neutrophils isolated from LF or HF mice. mean \pm s.e.m. k, Nitrotyrosine concentration (nM) in serum from LF or HF mice. LF, $n=7$ mice; HF, $n=6$ mice. mean \pm s.e.m.; two-tailed Student's t-test.

affinity for iron, thus preventing it from being accessed by bacterial siderophores ${ }^{26}$. Indeed, neutrophils from $L c n 2^{-/-}$mice exhibit impaired ROS production and reduced neutrophil extracellular DNA traps (NETs) in response to phorbol myristate acetate, and this is rescued by recombinant LCN2 protein $^{26}$. Similarly, neutrophils from $\mathrm{Ltf}^{-1-}$ mice exhibit impaired oxidative burst in response to phorbol myristate acetate ${ }^{27}$. These findings reinforce the concept that 'anemia of inflammation' is important to preserve neutrophil oxidative functions.

Neutrophil oxidative burst is a precursor to NETosis and MPO is required for the release of NETs ${ }^{28}$. We found increased NETs in $\mathrm{nCM}$, serum and lung tissue from obese versus lean mice (Fig. 6e-i).
Moreover, obese mice treated with catalase versus vehicle exhibited reduced NETosis in lung tissue via immunofluorescent costaining for MPO and citrullinated histone-H3 (H3cit) (Fig. 6j,k). Given that NETosis can cause collateral damage to normal tissues including the vasculature ${ }^{29}$, we tested whether NETosis affected BC extravasation by treating mice from the GIO model with a PAD4 inhibitor, GSK484, which prevents NET release ${ }^{30}$. BC cells were fluorescently labeled and injected via tail vein into WT or $o b / o b$ mice treated with the PAD4 inhibitor or vehicle (Fig. 6l). After $48 \mathrm{~h}$, tumor cell extravasation in $o b / o b$ mice was reduced in response to PAD4 inhibition compared to vehicle, with no effect in WT mice (Fig. $6 \mathrm{~m}$ ). This was not reproduced by treatment with DNase1 (Extended Data 
Fig. 6c-e), which degrades DNA scaffolds after NETs have already been released ${ }^{30}$. This suggests that preventing NETosis is necessary for therapeutic benefit, and that the harmful effects of NETs on BC extravasation have already taken place once NETs are released and may not be reversible.

Previous studies have shown that NETosis induced by inflammatory stimuli including lipopolysaccharide or tobacco exposure promotes the awakening of dormant metastases within the metastatic niche ${ }^{30}$. Since obesity is also an inflammatory stimulus, we tested whether obesity regulates metastatic dormancy through its effects on NETosis. We quantified the proportion of cancer cells with high CellTracker fluorescence intensity (low proliferation) in the GIO model following treatment with the PAD4 inhibitor versus vehicle. In accordance with previous studies ${ }^{30}$, we found that PAD4 inhibition increased the proportion of CellTracker ${ }^{\text {hi }}$ tumor cells in WT mice, however, only a trend was observed in $o b / o b$ mice (Fig. 6n,o). This suggests that the beneficial effects of PAD4 inhibition in the context of obesity may be specific to metastatic extravasation and only weakly promote metastatic dormancy. However, we cannot exclude the possibility that obesity-associated NETosis may regulate dormancy of established micrometastases, given that our model only spans $48 \mathrm{~h}$ (that is, recently extravasated cells). This is particularly relevant in light of recent data showing that associations between neutrophils and circulating tumor cells drive cell cycle progression during metastasis ${ }^{31}$. We found an increase in several factors known to underlie this phenotype in obese versus lean mice, including Tnfa, Osm, Illb, Csf1r3 and Tgfbr2 (Extended Data Fig. 6f).

Nos 2 knockout reduces cancer cell extravasation in obese hosts. We next tested the functional relevance of our observation that iNOS expression and activity are elevated in neutrophils from obese versus lean mice (Fig. 4d,j,k). We treated HMEC endothelial monolayers with $\mathrm{nCM}$ obtained from WT or $\mathrm{Nos}^{-/-}$mice enrolled on a SRD versus HF diet, and measured JAM1 levels by western blot. We found that JAM1 expression was restored in HMECs treated with nCM from HF Nos $2^{-1-}$ versus HF WT mice (Fig. 7a). To test whether iNOS regulates obesity-associated BC extravasation, we performed 48-h experimental metastasis assays in lean or obese WT versus Nos2 $2^{-1-}$ mice (Fig. 7b). Notably, Nos2 deletion caused an increase in body weight following HF diet, but not following SRD
(Extended Data Fig. 7a), consistent with previous work that showed HF Nos $2^{-/-}$mice exhibit elevated accumulation of epididymal and perirenal fat despite being protected from insulin resistance ${ }^{32}$. Despite their heavier body weight, $\mathrm{BC}$ extravasation was reduced in HF Nos $2^{-/-}$versus HF WT mice, but not in SRD controls (Fig. 7c). Immunofluorescence of lung tissues for MPO and $\mathrm{H} 3$ cit revealed reduced NETosis in HF Nos2 ${ }^{-1-}$ versus HF WT mice (Fig. 7d), in accordance with reduced serum hypochlorite (Fig. 7e). These data indicate that Nos 2 deletion phenocopies the effects of catalase in obese mice.

We next explored whether catalase treatment and Nos 2 deletion were synergistic. We isolated neutrophils from obese WT and Nos $2^{-/-}$mice, and generated $\mathrm{nCM}$ in the presence of catalase. We found elevated hypochlorite in nCM from obese versus lean mice, and this was reversed with either catalase treatment or Nos2 deletion. No synergistic effect was observed when Nos 2 deletion and catalase treatment were combined (Fig. 7f). This was functionally recapitulated using TEM assays in vitro (Fig. $7 \mathrm{~g}$ ) and BC extravasation assays in vivo (Fig. 7h). Given the role of iNOS and peroxynitrite on NETosis ${ }^{21}$, we performed immunofluorescence for MPO and $\mathrm{H} 3$ cit on lung tissues. We found no significant difference in NETosis in obese mice with Nos $2^{-1-}$ and catalase combined, compared with that in obese mice with $\mathrm{Nos}^{-/-}$or catalase alone(Fig. $7 \mathrm{i}$ ). These findings indicate that the combination of Nos 2 deletion and catalase treatment does not have synergistic effects, suggesting potential mechanistic redundancies between these two pathways.

The obesity-neutrophil axis is observed in patients with cancer with lung metastatic disease. To evaluate the translational relevance of our findings, we obtained lung metastasis samples from 22 patients with cancer with various primary tumors and body mass index (BMI) values ranging from 19.9 to 36.3 (Extended Data Fig. 8a), and performed imaging mass cytometry (IMC) using a panel of 35 multiplexed antibodies (Fig. 8a and Extended Data Fig. 8b,c). Of these, nine were used as cell lineage markers to assign tumor cells $\left(\mathrm{PanCK}^{+}\right)$, endothelial cells $\left(\mathrm{CD} 31^{+}\right)$, neutrophils $\left(\mathrm{MPO}^{+}\right)$, macrophages $\left(\mathrm{CD}^{+} 8^{+}\right), \mathrm{B}$ cells $\left(\mathrm{CD} 20^{+}\right)$, helper $\mathrm{T}$ cells $\left(\mathrm{CD}^{+} \mathrm{CD}^{+} \mathrm{CD} 8^{-}\right)$ and cytotoxic T cells $\left(\mathrm{CD}^{+} \mathrm{CD}^{-} \mathrm{CD}^{+}\right)$across a total of 172,580 cells. We performed $t$-distributed stochastic neighbor embedding $(t$-SNE) analysis to separate all metastasis samples into two dimensions

Fig. 5 | Neutrophil-ROS modulates vascular integrity and enhances BC extravasation during obesity in a reversible manner. a, Schematic illustration of in vivo extravasation assay corresponding to $\mathbf{c}$ and $\mathbf{f}$. $\mathbf{b}$, Serum hypochlorite in control C57BL6 mice treated with catalase or vehicle. $n=4$ mice per group; mean \pm s.e.m.; two-tailed Student's $t$-test. c, Fluorescence quantification of Py 230 cancer cell extravasation in lung tissue from LF or HF mice treated with catalase or vehicle. $\mathrm{LF}+$ veh, $n=5$ mice; $\mathrm{LF}+$ cat, $n=4$ mice; $\mathrm{HF}+$ veh, $n=5$ mice; HF + cat, $n=4$ mice; one-way ANOVA and Bonferroni multiple comparisons test. d, Fluorescence quantification of Py 230 cancer cell extravasation in lung tissue from LF or HF mice treated with vitamin $E$ ( $E$ ) or vehicle corresponding to Extended Data Fig. $5 b$. LF + veh, $n=4$ mice; LF + E, $n=3$ mice; HF + veh, $n=4$ mice; $H F+E, n=4$ mice; one-way ANOVA and Bonferroni multiple comparisons test. e, Serum hypochlorite in HF mice treated with vitamin E (vit E) or vehicle (veh). $n=3$ mice per group; mean \pm s.e.m.; two-tailed Student's t-test. f, Immunofluorescence analysis of JAM1 and CD31 colocalization in lung tissues isolated from LF or HF mice treated with catalase or vehicle. $\mathrm{LF}+$ veh, $n=6$ mice; $\mathrm{LF}+$ cat, $n=4$ mice; $\mathrm{HF}+$ veh, $n=8$ mice; $\mathrm{HF}+$ cat, $n=8$ mice; mean \pm s.e. m.; Kruskal-Wallis with Dunn's multiple comparisons test. $\mathbf{g}$, Fluorescence quantification of Py 230 cancer cell extravasation in lung tissue from WT or ob/ob mice treated with catalase or vehicle corresponding to Extended Data Fig. $5 \mathrm{~d}$. WT + veh, $n=9$ mice; WT + cat, $n=7$ mice; ob/ob + veh, $n=7$ mice; ob/ob+cat, $n=4$ mice; mean \pm s.e.m.; one-way ANOVA and Bonferroni multiple comparisons test. $\mathbf{h}$, Immunofluorescence analysis of JAM1 and CD31 colocalization in lung tissues isolated from WT or $o b / o b$ mice treated with catalase or vehicle. WT + veh, $n=7$ mice; WT + cat, $n=7$ mice; ob/ob + veh, $n=5$ mice; ob/ob+cat, $n=5$ mice; mean \pm s.e.m.; one-way ANOVA and Bonferroni multiple comparisons test. i, Py230 TEM across HUVEC monolayers treated with LF or HF bone-marrow $\mathrm{nCM} \pm$ catalase treatment. LF + veh, $n=7$ transwells; LF + cat, $n=4$ transwells; HF + veh, $n=8$ transwells; HF + cat, $n=4$ transwells; mean \pm s.e.m.; KruskalWallis with Dunn's multiple comparisons test. Transwells represent experimental replicates with $\mathrm{nCM}$ derived from individual mice. $\mathbf{j}$, In vitro dextran-FITC permeability assay depicting HUVEC monolayer permeability following treatment with LF or HF bone-marrow $\mathrm{nCM} \pm$ catalase. $\mathrm{LF}+$ veh, $n=5$ transwells; $\mathrm{LF}+$ cat, $n=6$ transwells; HF + veh, $n=5$ transwells; HF + cat, $n=6$ transwells; mean \pm s.e.m.; one-way ANOVA and Bonferroni multiple comparisons test. Transwells represent experimental replicates with $\mathrm{nCM}$ derived from individual mice. $\mathbf{k}$, Representative western blot of HMEC endothelial cells treated with LF or HF bone-marrow $\mathrm{nCM} \pm$ catalase for $24 \mathrm{~h}$. The displayed image is cropped; uncropped blot images are shown in the source data. $\beta$-actin was used as a loading control for normalization. Repeated three times with similar results. I, Py230 TEM across HMEC monolayers treated with LF or HF bone-marrow nCM. Catalase or methionine were added during or after nCM collection as indicated. LF, $n=5$ transwells; all other groups, $n=4$ transwells; mean \pm s.e.m.; one-way ANOVA with Bonferroni multiple comparisons test. All statistics shown are compared to HF. Transwells represent experimental replicates with $\mathrm{nCM}$ derived from individual mice. 
based on their expression of the 35 markers, and generated a heatmap based on the marker intensity across each population (Fig. 8b,c). Using this approach, we first examined the phenotype of cancer cells. Although we observed no difference in the overall number of cancer cells in $\mathrm{BMI}^{\text {high }}$ versus $\mathrm{BMI}^{\text {low }}$ patient samples (as expected, since tissue cores were sampled from within established metastases in both cases), cancer cells exhibited higher Ki67 and lower cleaved caspase 3 (CC3) staining intensity in $\mathrm{BMI}^{\text {high }}$ versus BMI ${ }^{\text {low }}$ samples (Fig. 8d). This is consistent with our finding that obesity contributes to accelerated tumor growth ${ }^{10}$ (Extended Data Fig. 1a-c), as well as literature linking obesity to increased cancer mortality ${ }^{4}$. Across all samples, we also found that JAM1 was highly expressed by tumor cells. However, in mice, the proportion of JAM1 ${ }^{+}$tumor cells was equal between lean and obese hosts (Extended Data Fig. 8d), and Jam1 knockdown in PyMT cells had no functional impact on TEM (Extended Data Fig. 8e). Therefore, although JAM1 is expressed by tumor cells, its expression within these cells is not affected by obesity and does not appear to regulate extravasation.

We next characterized the immune cell landscape within lung metastases. $t$-SNE analysis revealed unique differences in immune cell abundance and phenotype between $\mathrm{BMI}^{\text {high }}$ and $\mathrm{BMI}^{\text {low }}$ patient samples (Fig. 8b). Across all samples, the most abundant populations within the microenvironment included neutrophils, endothelial cells, macrophages and helper T cells. When we compared the phenotype of these cells in $\mathrm{BMI}^{\text {high }}$ and $\mathrm{BMI}^{\text {low }}$ tumors, we found that macrophages within $\mathrm{BMI}^{\text {high }}$ tumors exhibited reduced staining intensity for CD163 compared to those within BMI ${ }^{\text {low }}$ tumors, potentially indicative of an M1-like/proinflammatory phenotype (Fig. 8c and Extended Data Fig. 8f). These cells also expressed elevated Ki67 in $\mathrm{BMI}^{\text {high }}$ versus $\mathrm{BMI}^{\text {low }}$ tumors (Fig. $8 \mathrm{~d}$ ), which has been described for adipose tissue macrophages during obesity ${ }^{33}$. Moreover, $\mathrm{CD}^{+}$ helper $\mathrm{T}$ cells had an effector phenotype $\left(\mathrm{CD} 45 \mathrm{RO}^{\text {high }} \mathrm{CD} 45 \mathrm{RA}^{\text {low }}\right.$ a

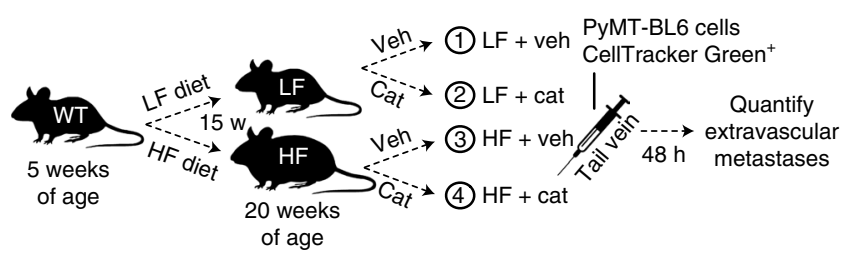

d

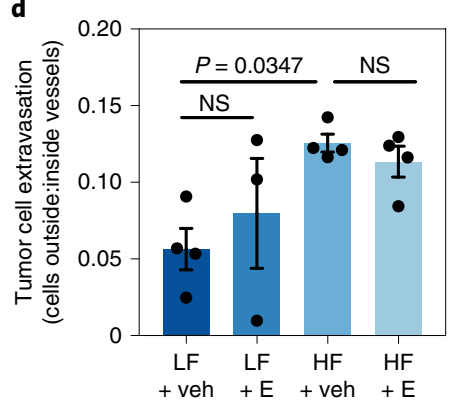

h
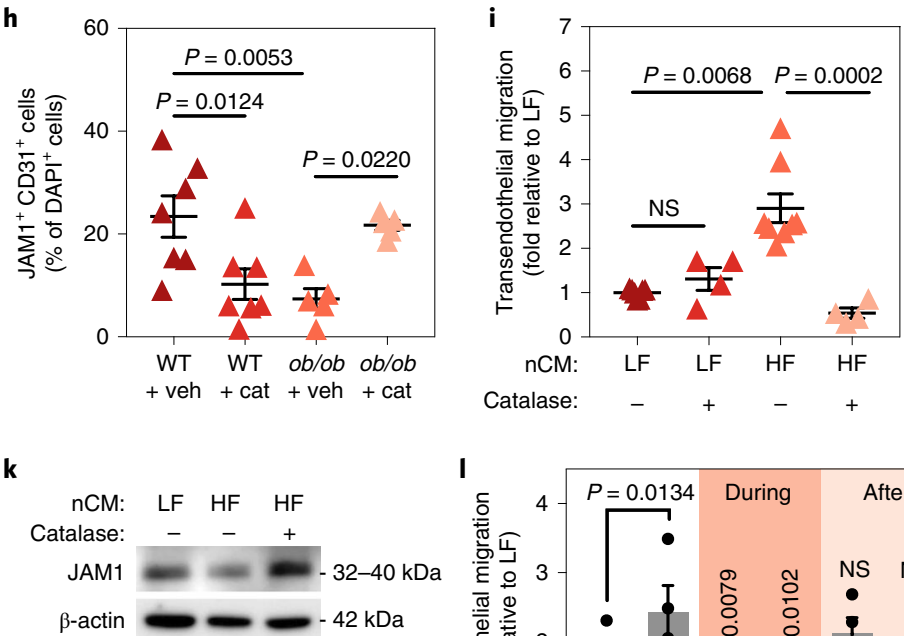
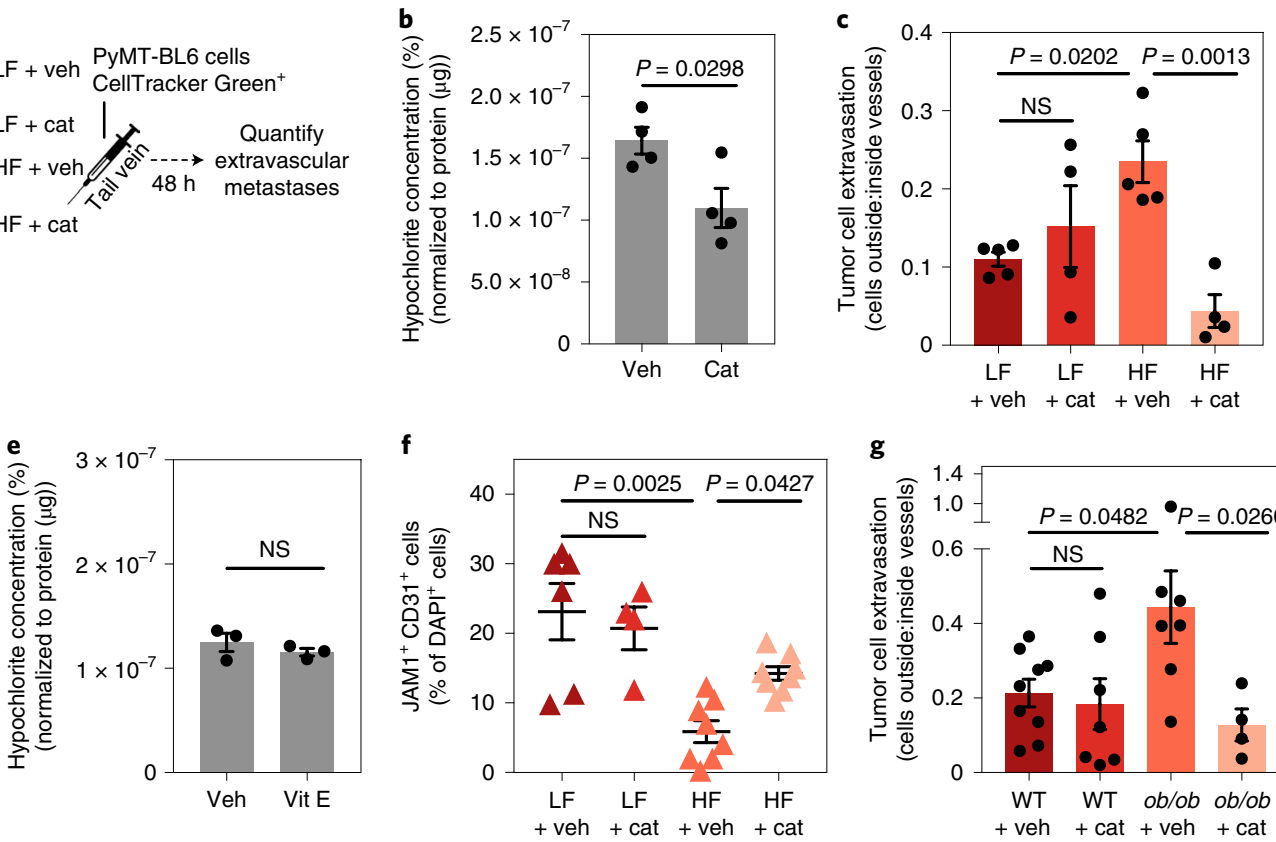

g

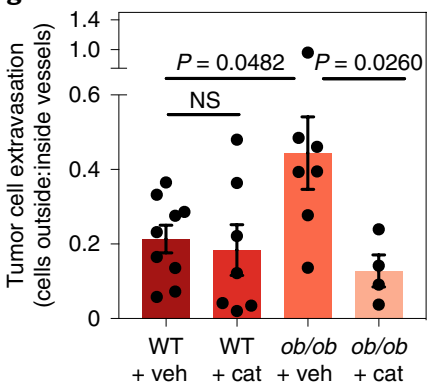

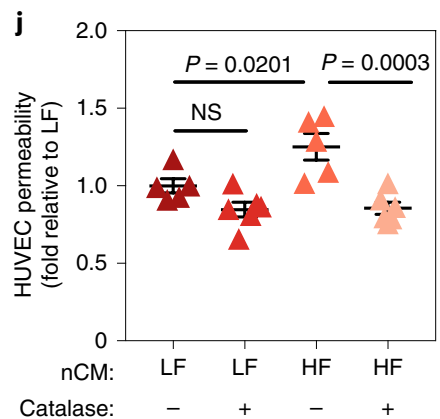

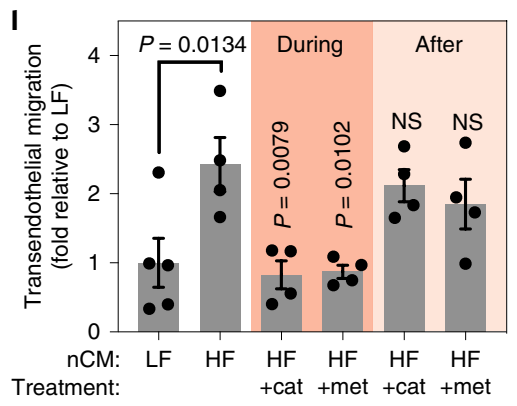




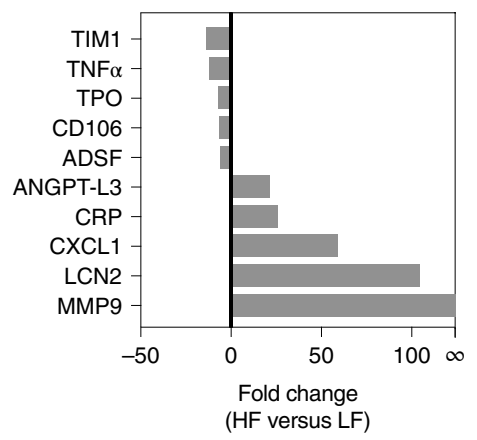

b

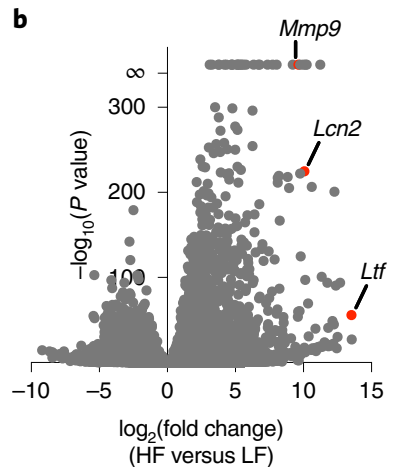

g

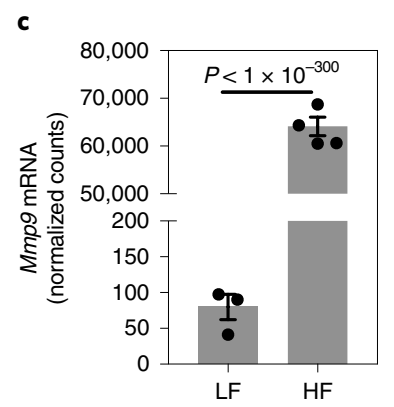

d
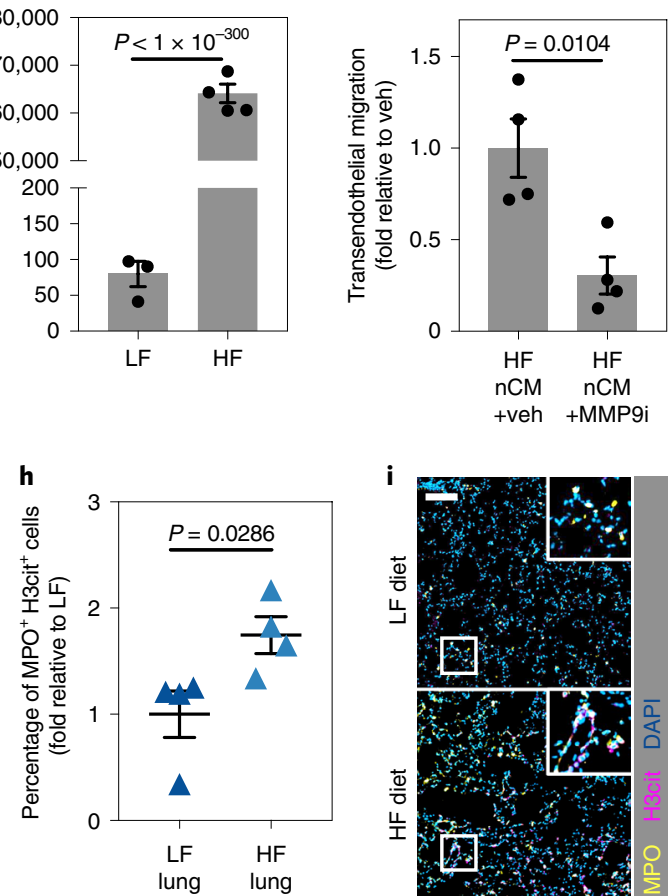

e

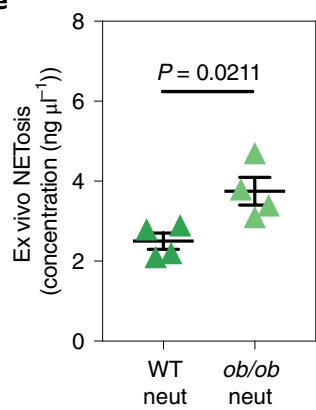

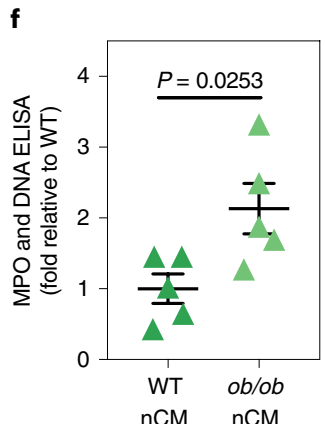
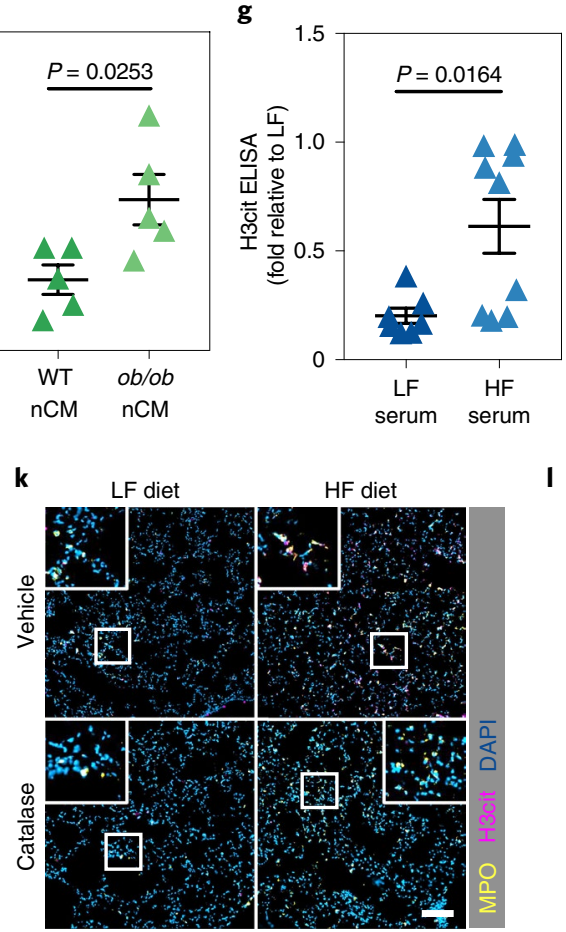

I
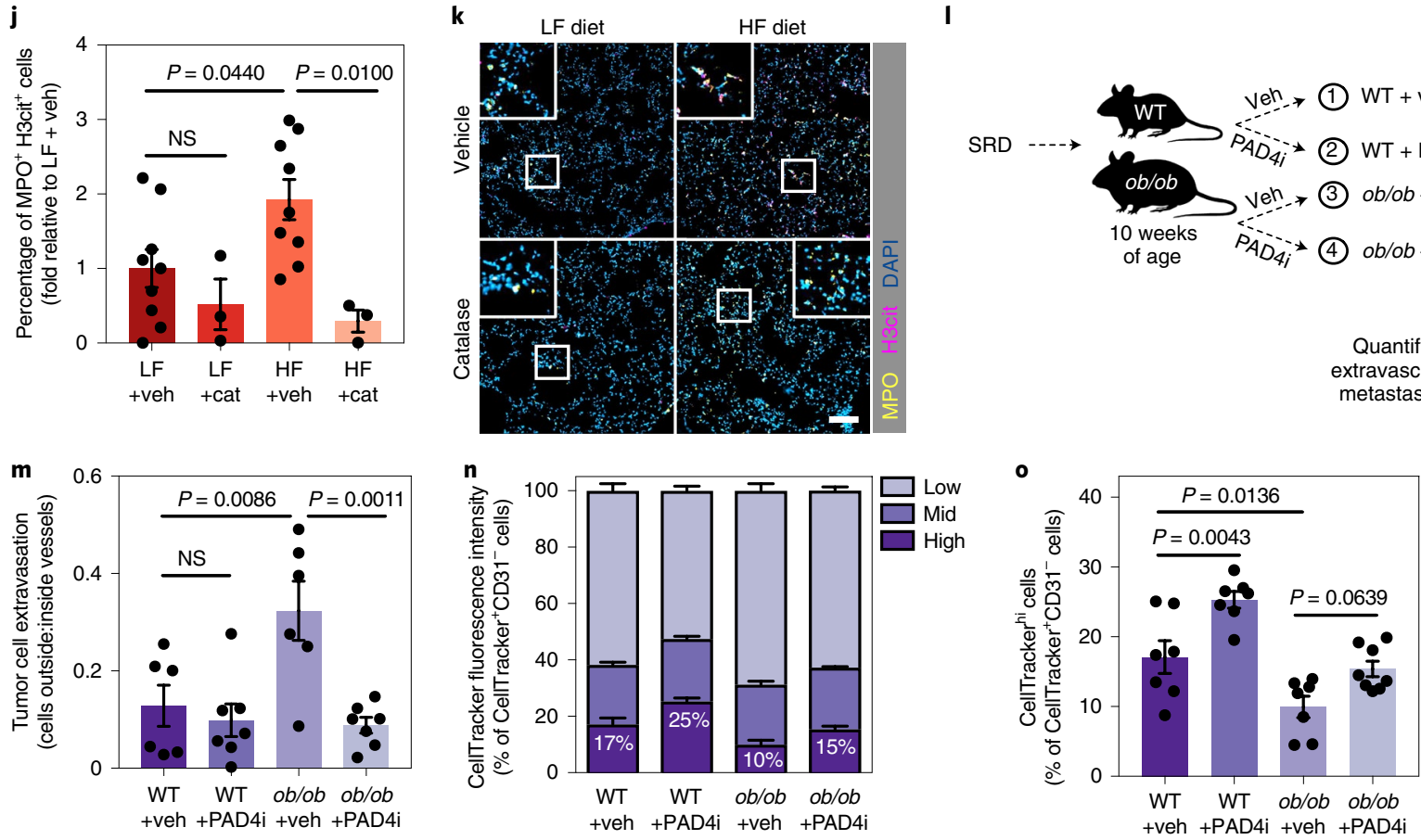

CCR7 ${ }^{\text {low }}$ ) coinciding with reduced Ki67 and elevated PD-1 expression, suggestive of exhaustion (Fig. $8 \mathrm{c}, \mathrm{d}$ ). This is consistent with emerging work linking enhanced immune checkpoint inhibitor efficacy in obese versus lean patients with cancer ${ }^{34,35}$.

We next focused on the phenotype of neutrophils. Consistent with previous observations in mice ${ }^{10}$, the number of $\mathrm{MPO}^{+}$neutrophils was increased in $\mathrm{BMI}^{\text {high }}$ versus $\mathrm{BMI}^{\text {low }}$ tumors (Fig. 8e). This coincided with a significant increase in the number of catalase ${ }^{-} \mathrm{MPO}^{+}$neutrophils in $\mathrm{BMI}^{\text {high }}$ versus $\mathrm{BMI}^{\text {low }}$ samples (Fig. 8f), which also had elevated staining intensity for iNOS, H3cit and MMP9 (Fig. 8d). Similarly, in BMI ${ }^{\text {high }}$ versus BMI ${ }^{\text {low }}$ tumors, we confirmed a significant increase in the number of catalase ${ }^{-} \mathrm{MMP9}^{+}$
$\mathrm{MPO}^{+}$neutrophils (Fig. 8g), and a trend for increased numbers of JAM1- endothelial cells (Fig. 8h). As a functional readout of these population dynamics, we performed immunofluorescent staining on patient samples for canonical markers of oxidative stress (chlorotyrosine, a marker of MPO-HOCl-mediated oxidation ${ }^{36}$ ) and NETosis (colocalization of MPO and H3cit). Consistent with our findings in mice, we found a positive correlation between the percentage of chlorotyrosine ${ }^{+}$cells and BMI within patient tumors (Fig. 8i,j), and a positive correlation between $\mathrm{MPO}^{+} \mathrm{H} 3 \mathrm{cit}^{+}$neutrophils and BMI within the tumor periphery (Fig. 8k,1). These data are consistent with our in vivo findings that obesity leads to enhanced oxidative burst and NETosis during metastasis. 
Fig. 6 | Obesity alters the secretory profile of neutrophils and enhances NETosis. a, Quantification of soluble proteins from a mouse cytokine array performed on conditioned media from lung neutrophils ( $\mathrm{nCM}$ ) isolated from LF or HF mice. Results are representative of two independent experiments. b, Volcano plot showing differentially expressed genes from RNA-seq of lung neutrophils isolated from HF versus LF mice. Mmp9, Lcn2 and Ltf are indicated (red dots). c, RNA-seq normalized counts of Mmp9 expression in lung neutrophils. A 799.87-fold increase in HF ( $n=4$ mice) versus LF ( $n=3$ mice); mean \pm s.e.m. mRNA, messenger RNA. d, Py230 TEM across HUVEC monolayers treated with LF or HF bone-marrow nCM \pm an MMP9 inhibitor for 24 h. $n=4$ transwells per condition representing experimental replicates with nCM from individual mice; mean \pm s.e.m.; two-tailed Student's $t$-test. e, Ex vivo spontaneous NETosis by bone-marrow neutrophils isolated from WT or ob/ob mice. $n=4$ mice per condition; mean \pm s.e.m.; two-tailed Student's t-test. f, ELISA for MPO and DNA to quantify NETs in WT or ob/ob bone-marrow nCM. $n=5$ mice per condition; mean \pm s.e.m.; two-tailed Student's t-test. g, ELISA for citrullinated histone-H3 (H3cit) in serum from LF or HF mice. LF, $n=7$ mice; HF, $n=9$ mice; mean \pm s.e.m.; two-tailed Mann-Whitney test. $\mathbf{h}$, Immunofluorescence analysis of MPO and H3cit in lung tissue from LF and HF mice. $n=4$ mice per condition; mean \pm s.e.m.; two-tailed MannWhitney test. $\mathbf{i}$, Representative immunofluorescence image for the data shown in $\mathbf{h}$. Scale bar, $100 \mu \mathrm{m}$. $\mathbf{j}$, Immunofluorescence analysis of MPO and H3cit in lung tissue from LF and HF mice treated with catalase or vehicle. LF + veh, $n=9$ mice; LF + cat, $n=3$ mice; HF + veh, $n=9$ mice; HF + cat, $n=3$ mice; mean \pm s.e.m.; one-way ANOVA with Bonferroni multiple comparisons test. $\mathbf{k}$, Representative immunofluorescence image for the data shown in $\mathbf{j}$. Scale bar, $100 \mu \mathrm{m}$. I, Schematic of the in vivo extravasation assay corresponding to $\mathbf{m}$. $\mathbf{m}$, Fluorescence analysis of Py 230 cancer cell extravasation in lung tissue from WT or ob/ob mice treated with PAD4i or vehicle, as depicted in j. WT + veh, $n=6$ mice; WT + PAD4i, $n=7$ mice; ob $/ o b+$ veh, $n=6$ mice; $o b / o b+P A D 4 i, n=7$ mice; mean \pm s.e.m.; one-way ANOVA with Bonferroni multiple comparisons test. $\mathbf{n}$, Distribution of CellTracker fluorescence intensity of tumor cells within the lungs of WT or ob/ob mice treated with a PAD4i or vehicle (high, less proliferative). WT + veh, $n=7$ mice; WT + PAD $4 i, n=7$ mice; $o b / o b+$ veh, $n=7$ mice; ob/ob +PAD4i, $n=8$ mice; mean \pm s.e.m.. o, Percentage of CellTracker ${ }^{\text {hi }}$ cells out of all CellTracker ${ }^{+}$cells that are CD31(outside the vessels), corresponding to the data in $\mathbf{n}$. WT + veh, $n=7$ mice; WT + PAD4i, $n=7$ mice; ob $/ o b+$ veh, $n=7$ mice; ob $/ o b+$ PAD4i, $n=8$ mice; mean \pm s.e.m.; one-way ANOVA with Bonferroni multiple comparisons test.

\section{Discussion}

Innate immunity and vascular physiology are two deeply coupled biological systems. We have discovered that obesity-associated neutrophilia compromises vascular integrity to facilitate BC extravasation into the lung parenchyma. This effect is dependent on neutrophil-produced ROS, which alters the secretory profile of neutrophils, triggers the release of NETs and disrupts endothelial junctions. The therapeutic value of antioxidants in cancer is complex. Although antioxidants can reduce cancer by mitigating DNA damage, paradoxically, they can also accelerate cancer by protecting cancer cells from ROS-mediated cytotoxicity ${ }^{37}$. Echoing this complexity, our data indicate that specific ROS scavengers, such as catalase, reduce metastasis in obese hosts, but not other antioxidants such as vitamin E. There are several interpretations of this finding. First, although antioxidants are often classified as a group, they represent a diverse family of molecules. For example, although vitamin $\mathrm{E}$ is a potent peroxyl radical scavenger, it is less efficient at scavenging $\mathrm{H}_{2} \mathrm{O}_{2}$ and nonradical oxidants such as $\mathrm{HOCl}^{23}$. Second, myeloid cells are a major source of ROS in the tumor microenvironment therefore, the efficacy of antioxidant supplementation may be partially dependent on microenvironmental composition and whether neutrophils or other granulocytes are abundant (as in obesity). Finally, in certain contexts, antioxidants may help balance pathological
ROS levels back to homeostatic baseline. This is exemplified by the beneficial effects of antioxidants on cancer prevention in poorly nourished populations ${ }^{38}$. Moreover, obesity is associated with systemic oxidative stress in humans ${ }^{39}$, and $10 \%$ weight loss in morbidly obese women reduces urinary $\mathrm{F} 2$-isoprostane-M (marker of oxidative stress $)^{40}$. Therefore, general antioxidant supplementation in patients with cancer should be approached with caution, as specific interventions that balance ROS levels may be more appropriate.

Although our study suggests a potential therapeutic use for catalase, this enzyme is not used in patients. Future investigations examining clinically approved antioxidants in the context of obesity are warranted, such as $\mathrm{N}$-acetylcysteine-a precursor to glutathione and a potent scavenger of $\mathrm{H}_{2} \mathrm{O}_{2}$ and $\mathrm{HOCl}^{41}$. However, obesity not only increases ROS in neutrophils, it also increases neutrophil numbers ${ }^{10,42,43}$. Therefore, therapeutic approaches that normalize neutrophil frequency in obese individuals, such as weight loss ${ }^{10,42}$ or inhibitors of adipose-stimulated granulopoiesis (for example, IL-1R antagonists $)^{42}$, may complement ROS-targeted approaches. Our study has broader clinical implications beyond therapy. We have identified a serum factor $(\mathrm{HOCl})$ in our mouse models that is indicative of neutrophil oxidative stress and coincides with metastatic phenotypes. Whether $\mathrm{HOCl}$ can be used as a biomarker in patients remains unknown. Given the limitations of BMI to accurately

Fig. 7 | Nos2 knockout reduces cancer cell extravasation in obese hosts. a, Representative western blot of HMEC endothelial cells treated with nCM collected from LF WT, HF WT or HF Nos2 $2^{-/-}$bone-marrow neutrophils. The displayed image is cropped; uncropped blot images are shown in the source data. $\beta$-actin was used as a loading control for normalization. Repeated three times with similar results. $\mathbf{b}$, Schematic of in vivo extravasation assay corresponding to c-e. c, Fluorescence quantification of Py230 cancer cell extravasation in lung tissue from WT or Nos2 ${ }^{-/-}$mice enrolled on SRD or HF diet, as in b. SRD WT, $n=5$ mice; SRD Nos $2^{-/-}, n=5$ mice; HF WT, $n=9$ mice; HF Nos2 $2^{-1-}, n=5$ mice; mean \pm s.e.m. Note that SRD and HF cohorts were performed as independent experiments, therefore a two-tailed Student's $t$-test was used for the indicated comparisons. d, Immunofluorescence analysis of MPO and H3cit in lung tissue from WT or Nos2-/- mice enrolled on SRD or HF diet. $n=5$ mice per group; mean \pm s.e.m.; two-tailed Student's $t$-test for the indicated comparisons. e, Serum hypochlorite from WT or Nos2 ${ }^{-1-}$ mice enrolled on SRD or HF diet. $n=5$ mice per group; mean \pm s.e.m.; two-tailed Student's $t$-test for the indicated comparisons. f, Serum hypochlorite from LF versus HF mice, or HF mice with catalase treatment and/or Nos2 deletion. LF WT, $n=3$ mice; HF WT, $n=5$ mice; HF WT + cat, $n=3$ mice; HF Nos2 $2^{-\prime-}, n=4$ mice, HF Nos2 ${ }^{-\prime-}+$ cat, $n=4$ mice; mean \pm s.e.m.; one-way ANOVA with Bonferroni multiple comparisons test. $\mathbf{g}$, Py230 TEM across HMEC monolayers treated with bone-marrow nCM obtained from LF versus HF mice, or HF mice with catalase treatment or Nos2 deletion. LF WT, $n=4$ transwells; HF WT, $n=6$ transwells; HF WT + cat, $n=4$ transwells; HF Nos2 ${ }^{-/-}$, $n=4$ transwells, HF Nos2 ${ }^{-1-}+$ cat, $n=4$ transwells; mean \pm s.e.m.; one-way ANOVA with Bonferroni multiple comparisons test. Transwells represent experimental replicates with $\mathrm{nCM}$ derived from individual mice. $\mathbf{h}$, Fluorescence quantification of Py 230 cancer cell extravasation in lung tissue from LF or HF WT or Nos2 ${ }^{-1-}$ mice treated with catalase or vehicle. LF WT, $n=5$ mice; HF WT, $n=7$ mice; HF WT + cat, $n=4$ mice; HF Nos2 ${ }^{-/-}, n=7$ mice, HF Nos2 $2^{-1-}+$ cat, $n=7$ mice; mean \pm s.e.m.; one-way ANOVA with Bonferroni multiple comparisons test. $\mathbf{i}$, Immunofluorescence analysis of MPO and $\mathrm{H} 3$ cit costaining (NETs) in lung tissue from LF or HF WT or Nos2-/- mice treated with catalase or vehicle. LF WT, $n=5$ mice; HF WT, $n=7$ mice; HF WT + cat, $n=4$ mice; HF Nos2 ${ }^{-/-}, n=8$ mice, HF Nos $2^{-/-}+$cat, $n=8$ mice; mean \pm s.e.m.; one-way ANOVA with Bonferroni multiple comparisons test. 
pinpoint patients with metabolic syndrome who have a heightened susceptibility to cancer mortality, identifying serum factors that can be measured in patients has strong therapeutic value.

Although tumors can promote the accumulation of neutrophils and their abundance is generally associated with poor survival ${ }^{44,45}$, previous work demonstrates that obesity is sufficient on its own to promote neutrophilia and that this is exacerbated by cancer ${ }^{10}$. Our study mechanistically extends these observations by demonstrating that lung neutrophils are functionally distinct in obese versus lean hosts. It remains unclear if this is a result of population enrichment or differences in differentiation. For example, obesity may alter granulopoiesis and neutrophil maturation within bone marrow, as adipose tissue can stimulate myelopoiesis in the obese state ${ }^{42}$ and myeloid cell-derived ROS is known to stimulate bone-marrow progenitor expansion during emergency granulopoiesis ${ }^{46}$. Alternatively, obesity may enrich for granulocyte subsets in lung with a heightened a
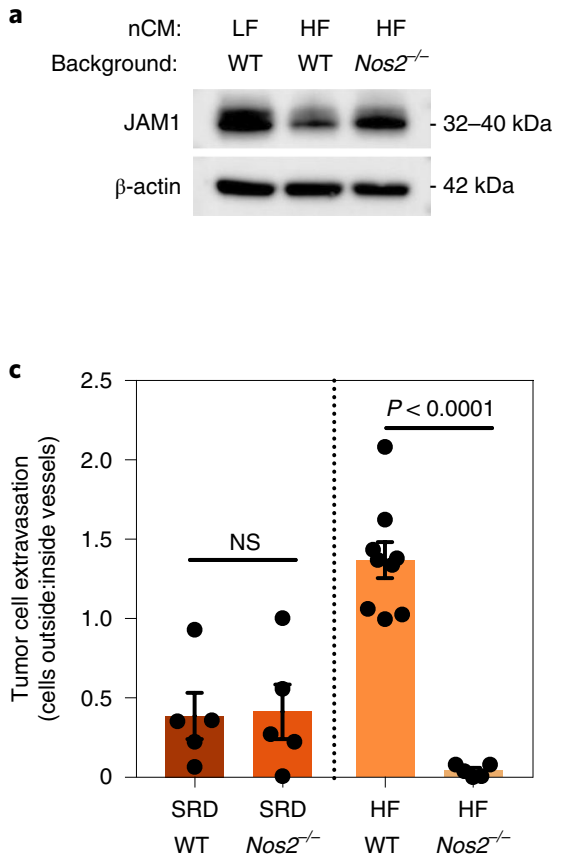

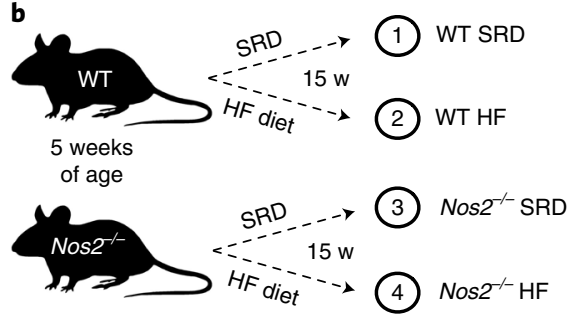

PyMT-BL6 cells CellTracker Green ${ }^{+}$

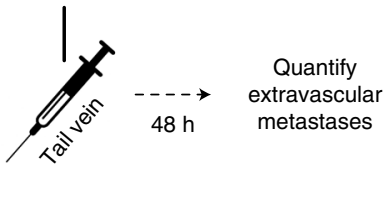

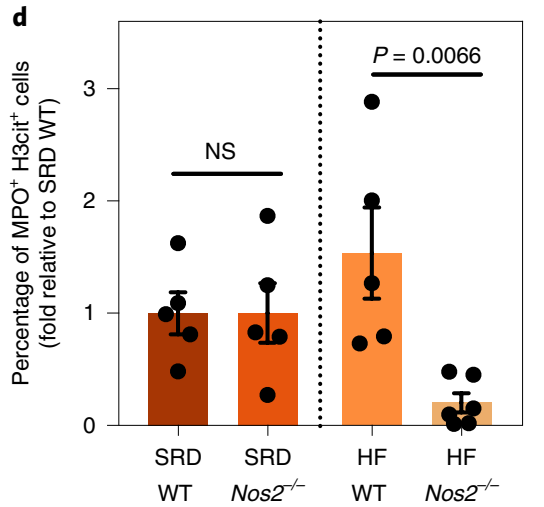

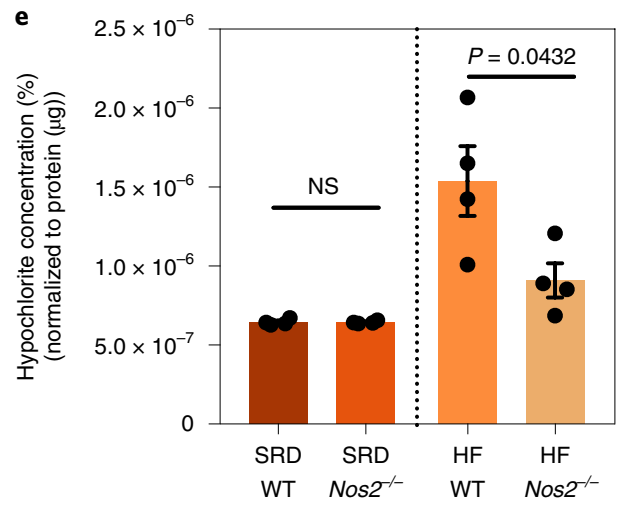

$\mathbf{f}$

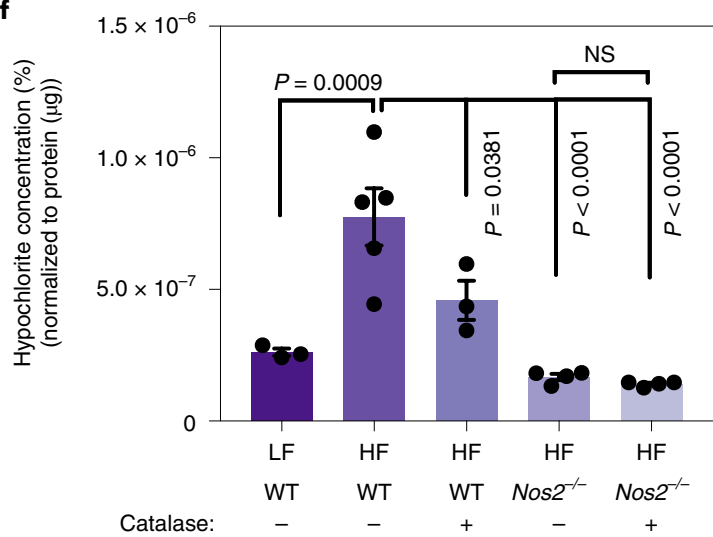

h

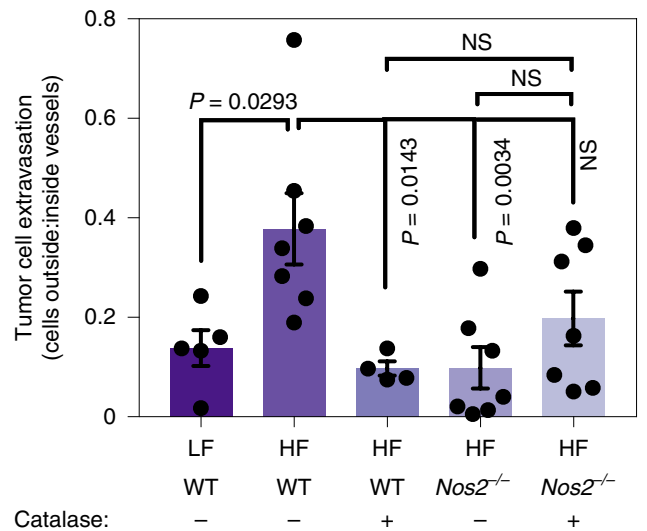

g
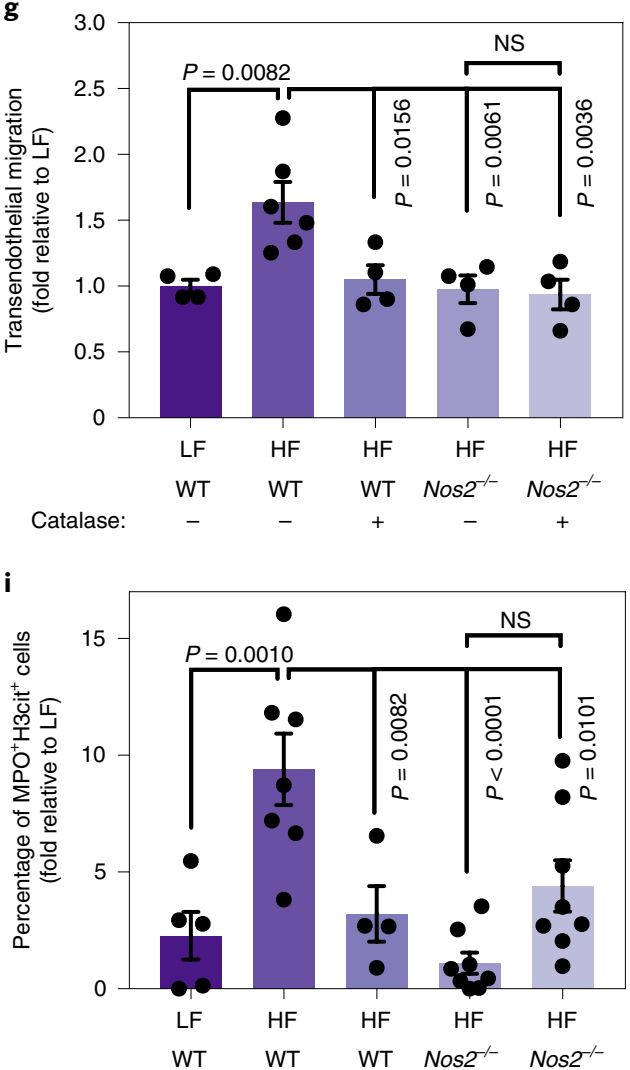

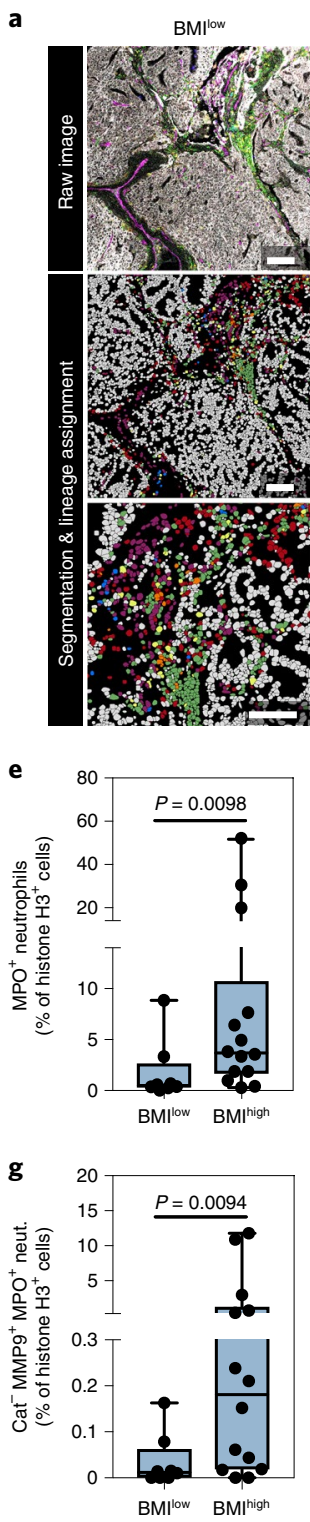

i

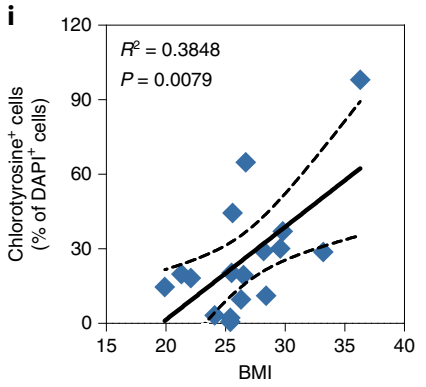

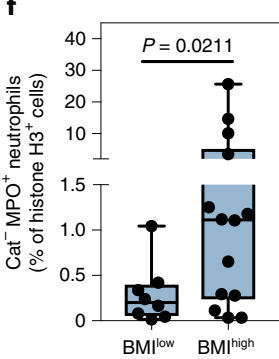
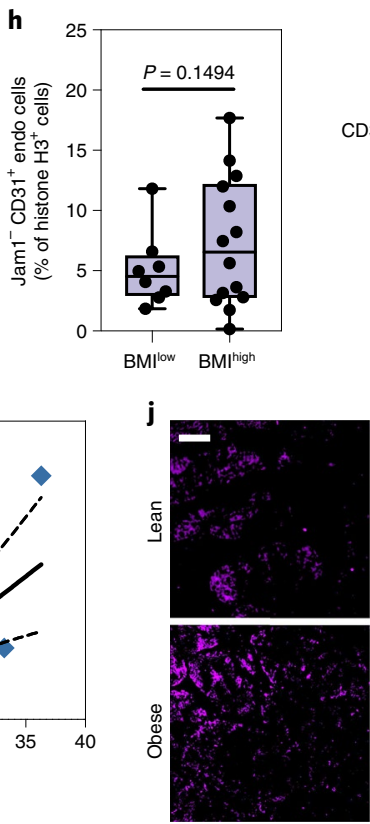

d b
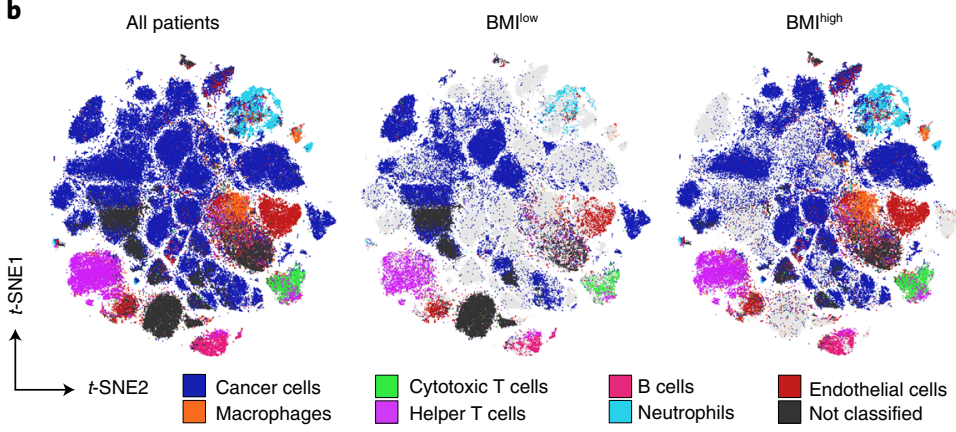

C
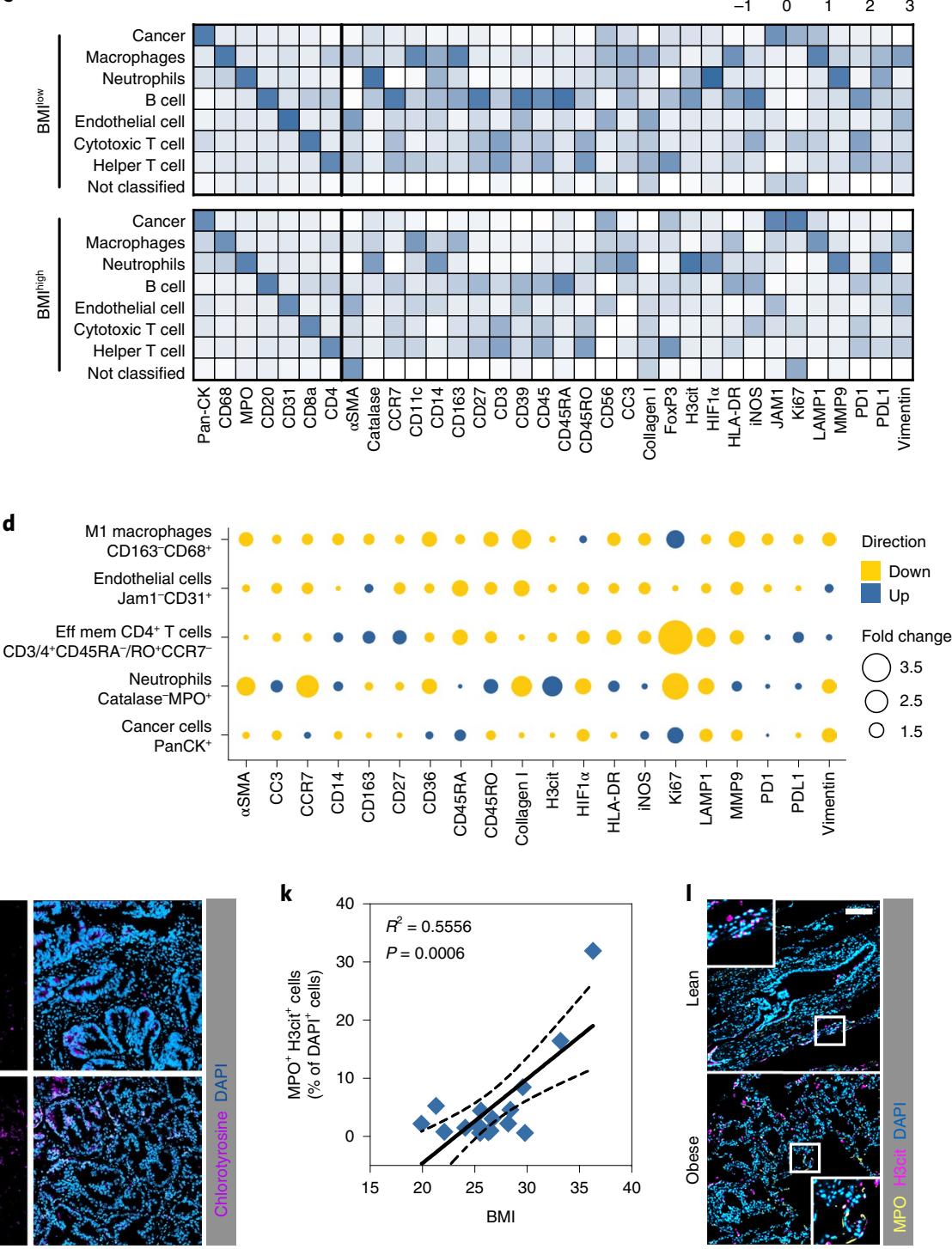

$\mathbf{k}$

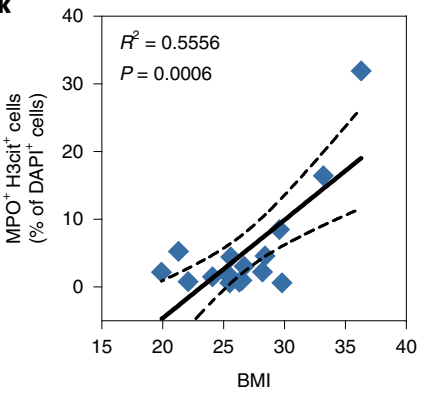

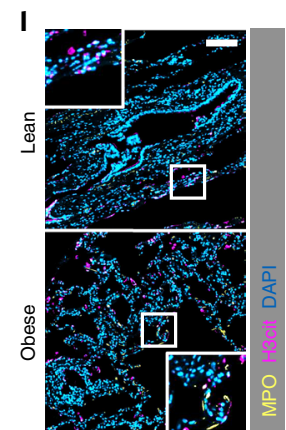

capacity for ROS production and vascular injury, such as aged neutrophils ${ }^{47}$ (Cxcr2 is elevated by 2,415 -fold in HF versus LF neutrophils in our model; $\left.P<1 \times 10^{-300}\right)$, ICAM-1 $1^{\text {hi }}$ neutrophils ${ }^{48}$ or immunosuppressive myeloid cells that rely on iNOS ${ }^{49}$. Indeed, studies have shown that obesity increases $\mathrm{Grl}^{+}$myeloid cells in peripheral tissues and accelerates progression of $4 \mathrm{~T} 1 \mathrm{BC}^{50,51}$. Thus, our data add to a growing literature on neutrophil heterogeneity and diversity in health and disease ${ }^{52}$.

We found that obesity-associated neutrophils promote vascular permeability through enhanced MPO-HOCl. Consistently, 
Fig. 8 | The obesity-neutrophil axis is observed in patients with cancer with lung metastatic disease. a, Representative IMC images from BMlow ( $n=8$ patients) and BMI high ( $n=14$ patients) lung metastasis samples. Unprocessed images (top) with corresponding processed images with lineage assignment (bottom) are shown. Scale bar, $100 \mu \mathrm{m}$. b, $t$-SNE analysis displaying cell population clusters across all patient samples (left, $n=22$ patients), $\mathrm{BMI}^{\text {low }}$ (middle, $n=8$ patients) and BMl high (right, $n=14$ patients). Specific gated cell populations of interest are indicated in the legend. $\mathbf{c}$, Heatmap of average signal intensity of each marker within the indicated cell populations (corresponding to those in $\mathbf{b}$ ). Scale bar represents the $z$ score for signal intensity relative to all samples in a given channel. $\mathbf{d}$, Balloon plot representing the difference in average signal intensity between BMI high versus BMllow samples in the indicated cell populations. e, Average frequency of MPO+ neutrophils in BMl low $(n=8$ patients) and BMligh ( $n=14$ patients) samples. Box, median \pm interquartile range; whiskers, min-max; all datapoints shown; one-tailed Mann-Whitney test. f, Average frequency of catalase- MPO $^{+}$ neutrophils in BMI low $\left(n=8\right.$ patients) and BMI ${ }^{\text {high }}(n=14$ patients) samples. Box, median \pm interquartile range; whiskers, min-max; all datapoints shown; one-tailed Mann-Whitney test. $\mathbf{g}$, Average frequency of catalase- MMP9+ $\mathrm{MPO}^{+}$neutrophils in BMl low $\left(n=8\right.$ patients) and BMl ${ }^{\text {high }}(n=14$ patients) samples. Box, median \pm interquartile range, whiskers, min-max; all datapoints shown; one-tailed Mann-Whitney test. $\mathbf{h}$, Average frequency of Jam1- CD31+ endothelial cells in BMlow $\left(n=8\right.$ patients) and BMI ${ }^{\text {high }}(n=14$ patients) samples. Box, median \pm interquartile range; whiskers, min-max; all datapoints shown; one-tailed Student's $t$-test. $\mathbf{i}$, Immunofluorescence analysis of percentage of chlorotyrosine ${ }^{+}$cells in patient lung metastases as a function of BMI. $n=17$ patients; Pearson's correlation, $R^{2}=0.3629, P=0.0079$. j. Representative immunofluorescence image for the data shown in $\mathbf{i}$. Scale bar, $100 \mu \mathrm{m}$. $\mathbf{k}$, Immunofluorescence analysis of percentage of $\mathrm{MPO}^{+} \mathrm{H}$ cit ${ }^{+}$cells (NETs) adjacent to lung metastases in patients as a function of BMI. $n=17$ patients; Pearson's correlation, $R^{2}=0.5556, P=0.0006$. I, Representative immunofluorescent image for the data shown in $\mathbf{k}$. Scale bar, $100 \mu \mathrm{m}$.

surgery, or infection ${ }^{30,58,59}$. Our study demonstrates that obesity is also sufficient to induce NETosis in blood and lungs, consistent with data in obese humans showing elevated ROS in peripheral neutrophils ${ }^{60}$. However, it remains unknown whether the location of NETs affects their function. For example, it is possible that blood-NETosis in obese mice regulates vascular dysfunction, while lung-NETosis regulates metastatic seeding, dormancy and/or proliferation. Whether patients living with obesity and cancer would benefit from NET-targeted therapies remains uncertain, as these agents are only starting to be tested clinically in patients with severe COVID-19 (for example, the DISCONNECT-1 phase 1 trial).

We have shown that obesity impairs lung vascular function by altering junctional adhesions. This is consistent with data from acute respiratory distress syndrome (ARDS), where obesity increases leukocyte adhesion molecules (E-selectin and ICAM-1) and reduces junctional adhesions (VE-cadherin and $\beta$-catenin) in the lung endothelium, predisposing to vascular injury ${ }^{61}$. This is reversed via adiponectin repletion in obese mice, coinciding with reduced serum leptin, triglycerides and free fatty acids. It remains unknown whether adiponectin can similarly mitigate neutrophilia and/or ROS in our model; however, it has been shown to regulate neutrophil and monocyte oxidative bursts through its anti-inflammatory properties $^{62,63}$, and pioglitazone (a clinically approved PPARy agonist for type 2 diabetes) can induce adiponectin in mice and humans ${ }^{64}$. In addition to ARDS, obesity is linked to several lung inflammatory conditions, including chronic obstructive pulmonary disease and asthma, despite the absence of adipocytes locally. This is likely due to the systemic effects of inflamed adipose tissue ${ }^{9,65}$.

Ultimately, despite strong clinical evidence linking obesity with cancer mortality, it is not known how to improve cancer outcomes in patients living with obesity as mechanistic insight is lacking. Our study addresses this knowledge gap by shedding light on how obesity enhances specific aspects of the metastatic cascade through its effects on neutrophils. Since depleting neutrophils in patients with cancer is not feasible due to risk of infection, our findings demonstrate how neutrophil function can be balanced to treat disease progression. Given the rising incidence of obesity globally, our findings have translational relevance for a substantial proportion of the adult population.

\section{Methods}

Cell lines. Pooled primary HUVECs were purchased from Lonza, and cultured in Medium 199 with $1 \%$ penicillin and streptomycin (P/S), $0.2 \%$ bovine brain extract, $5 \mathrm{ng} \mathrm{ml}^{-1}$ recombinant epidermal growth factor (rhEGF), $10 \mathrm{mM}$ L-glutamine, $0.75 \mathrm{U} \mathrm{ml}^{-1}$ heparin sulfate, $1 \mu \mathrm{g} \mathrm{ml}^{-1}$ hydrocortisone, $50 \mu \mathrm{g} \mathrm{ml}^{-1}$ ascorbic acid and 2\% fetal bovine serum (FBS) (Endothelial Cell Growth Kit-BBE, ATCC). HMECs were a gift from P. Siegel (originally from ATCC), and cultured in Medium MCDB131 (without L-glutamine) with $1 \% \mathrm{P} / \mathrm{S}, 10 \mathrm{ng} \mathrm{ml}^{-1} \mathrm{EGF}$,
$1 \mu \mathrm{g} \mathrm{ml}^{-1}$ hydrocortisone, $10 \mathrm{mM}$ glutamine and 10\% FBS. Py230 BC cells were purchased from ATCC, originally isolated from MMTV-PyMT mice on C57BL6 background ${ }^{66,67}$. Py 230 cells were cultured in Ham's F-12K (Kaighn's basal medium) with $1 \% \mathrm{P} / \mathrm{S}, 0.1 \% \mathrm{MITO}^{+}$and 5\% FBS. E0771 BC cells were purchased from $\mathrm{CH} 3$ Biosystems, originally isolated from a spontaneous breast tumor in C57BL6 mice ${ }^{68}$. E0771 cells were cultured in Roswell Park Memorial Institute medium 1640 with 1\% $\mathrm{P} / \mathrm{S}$ and $10 \%$ FBS. MDA-MB-231 BC cells were purchased from ATCC, originally obtained from a human mammary gland adenocarcinoma. MDA-MB-231 cells were cultured in Leibovitz's L-15 medium with $1 \% \mathrm{P} / \mathrm{S}$ and $10 \%$ FBS.

Pharmacological and biological reagents. Depletion of neutrophils in vivo was performed using an anti-Grl antibody (clone RB6-8C5), $6 \mathrm{mg} \mathrm{kg}^{-1}$ body weight, administered intraperitoneally (i.p.) 3 and $1 \mathrm{~d}$ before tumor cell injections (rat IgG2b isotype control, Tonbo Biosciences) $)^{10,50}$. Flow cytometry confirmed depletion of neutrophils in blood, spleen and lung for $3 \mathrm{~d}$ at a minimum ${ }^{10}$. For a more specific strategy to deplete neutrophils in vivo, we used anti-Ly6G (clone $1 \mathrm{~A} 8$ ) with a mouse IgG2a antibody against rat kappa immunoglobulin (clone MAR 18.5), both at a dose of $4 \mathrm{mg} \mathrm{kg}^{-1}$ body weight (rat IgG2a isotype control, BioXcell $)^{18}$. Anti-Ly6G was administered i.p. 2 d before tumor cell injection, followed by anti-IgG $1 \mathrm{~d}$ before tumor cell injection. Neutrophil depletion was validated by flow cytometry $3 \mathrm{~d}$ later, at the 48 -h metastasis endpoint. Neutrophils were quantified as Live/Dead Aqua- $\mathrm{CD} 45^{+} \mathrm{CD} 11 \mathrm{~b}^{+}$Ly6C ${ }^{\text {lo }}$, and side scatter was used to further separate Ly6C ${ }^{\text {lo }}$ populations instead of Ly6G 1A8. Catalase-PEG was administered i.p. at $10,000 \mathrm{U} \mathrm{kg}^{-1}$ (Sigma) ${ }^{69}$. This conjugated form of catalase was used because it prolongs catalase activity in vivo (50-fold increase in retention time in plasma compared to unconjugated catalase $)^{22,69}$. On the day of tumor cell injection, catalase-PEG was administered directly following tumor cell injection and then again $3 \mathrm{~h}$ later; mice were subsequently treated once daily. Catalase was used in vitro at a working concentration of $60 \mathrm{U} \mathrm{ml}^{-1}$ (Sigma) ${ }^{70}$. Vitamin E ( $\alpha$-tocopherol) was administered i.p. at $20 \mathrm{mg} \mathrm{kg}^{-1}$ (Sigma) ${ }^{71}$. On the day of tumor cell injection, $\alpha$-tocopherol was administered directly following tumor cell injection and then again $3 \mathrm{~h}$ later; mice were subsequently treated once daily. Inhibition of PAD4 was performed using GSK484 (hydrochloride, Cayman Chemical) at $20 \mathrm{mg} \mathrm{kg}^{-1}$ as previously described ${ }^{30}$. On the day of tumor cell injection, mice were treated three times $(30 \mathrm{~min}$ before tumor cell injection, $3 \mathrm{~h}$ after tumor cell injection and $6 \mathrm{~h}$ after tumor cell injection). Mice were subsequently treated once daily. Digestion of NET DNA was performed using DNase1, administered i.p. once daily at $15,000 \mathrm{U} \mathrm{kg}^{-1}$ (Roche $)^{30}$. L-Methionine was used in vitro at a working concentration of $1 \mathrm{mM}$ (Sigma).

Animal models. Mice were housed in pathogen-free conditions. Protocols were reviewed and approved by McGill University Animal Care committee and conformed to standards by Canadian Council on Animal Care.

DIO model. The DIO model was used as previously described ${ }^{10}$. Five-week-old female C57BL6 mice (Jackson Laboratory) were enrolled on HF (60\% fat, 20\% protein, $20 \%$ carbohydrate; Research Diets D12492I) or LF ( $0 \%$ fat, $20 \%$ protein, $70 \%$ carbohydrate; Research Diets D12450BI) irradiated isocaloric diet for 15 weeks. Weight was monitored once weekly. DIO mice were used at 20 weeks of age (average weight, $36.0 \mathrm{~g}$ compared to $23.6 \mathrm{~g}$ in LF controls).

GIO model. The GIO model was used as previously described ${ }^{10}$. Female age-matched WT or $o b / o b$ mice (Jackson Laboratory) were maintained on a SRD. $\mathrm{Ob} / \mathrm{ob}$ mice gain weight rapidly from hyperphagia as a consequence of leptin deficiency. GIO mice were used at 10 weeks of age (average weight, $46.1 \mathrm{~g}$ compared to $18.8 \mathrm{~g}$ in WT controls). 
MMTV-PyMT model. We used MMTV-PyMT mice on a C57BL6 background (Jackson Laboratory) as these mice develop tumors later than those on a FVB/n background $^{72}$ (sufficient time for weight gain). Five-week-old female C57BL6 MMTV-PyMT mice were enrolled on HF or LF irradiated isocaloric rodent diet until endpoint ( 25 weeks of age, or maximum tumor volume of $2 \mathrm{~cm}^{3}$ ). Weight was monitored once weekly and tumor volume once weekly, once it was palpable. At endpoint, lung tissues were stained with hematoxylin and eosin, scanned using AperioScanner and Spectrum software analysis was used to quantify the fraction of lung area occupied by metastases in serial sections.

Nos $2^{-/-}$mice. Five-week-old female C57BL6 Nos $2^{-/-}$mice (Jackson Laboratory) were enrolled on a HF or SRD ( $16 \%$ fat, $24 \%$ protein, $60 \%$ carbohydrate) for 15 weeks. Weight was monitored once weekly. Nos $2^{-1-}$ mice were used for experiments at 20 weeks of age (average weight, $42.3 \mathrm{~g}$ for HF, compared to $21.02 \mathrm{~g}$ for SRD).

Serum isolation from mice. Submandibular bleeds were performed via Goldenrod lancet into Eppendorf tubes, and allowed to clot at room temperature for $20 \mathrm{~min}$. Samples were centrifuged at 2,000 r.p.m., $4^{\circ} \mathrm{C}$ for $20 \mathrm{~min}$. Supernatant was transferred to a polypropylene tube and $\geq 3$ individual mouse samples were pooled and stored at $-80^{\circ} \mathrm{C}$ for downstream applications. Serum was not heat inactivated unless indicated otherwise.

In vivo extravasation assay and quantification. BC cells (Py230) were labeled with green fluorescent CellTracker (Vybrant CFDA SE Cell Tracer Kit, Invitrogen; 1:1,500 dilution in $1 \times \mathrm{PBS})$. Then $1 \times 10^{6} \mathrm{BC}$ cells were injected via tail vein, and after $48 \mathrm{~h}$, mice were euthanized and lungs were perfused through the trachea with $4 \%$ paraformaldehyde (PFA) (Santa Cruz). Lungs were collected and incubated for $24 \mathrm{~h}$ in $4 \%$ PFA at $4{ }^{\circ} \mathrm{C}$, followed by $24 \mathrm{~h}$ in $30 \%$ sucrose at $4{ }^{\circ} \mathrm{C}$. Tissues were embedded and frozen in optimal cut temperature compound (Tissue-Tek, Sakura), and cryosectioned for immunofluorescence. Fluorescence in whole-lung sections was visualized for CD31 immunostaining and for CellTracker ${ }^{+}$tumor cells using an Axio Scan.Z1 (Zeiss). HALO Image Analysis Software v.3.0.311.195 (Perkin Elmer) was used to quantify fluorescence. Extravasation was quantified as the ratio of cancer cells outside the vessels $\left(\right.$ CellTracker $\left.{ }^{+} \mathrm{CD} 31^{-}\right)$versus inside the vessels $\left(\right.$ CellTracker $\left.{ }^{+} \mathrm{CD} 31^{+}\right)$. One randomly selected serial section of the left lobe per mouse was used for these analyses; additional serial sections were used for complementary immunofluorescence staining (for example, JAM1, MPO, H3cit and so on) and the right lobe was used for flow cytometry or primary culture.

Immunofluorescent staining of tissues. Immunofluorescent staining was performed as described ${ }^{73}$. Briefly, $10-\mu \mathrm{m}$ sections were thawed and dehydrated at room temperature, then rehydrated in $1 \times$ PBS before staining. Tissues were blocked with Dako blocking reagent ( $1 \mathrm{~h}$, room temperature; Agilent). Primary antibodies were diluted in Dako antibody diluent and incubated overnight at $4^{\circ} \mathrm{C}$. Tissues were rinsed in $1 \times$ PBS, incubated for $1 \mathrm{~h}$ at room temperature with AlexaFluor secondary antibodies (1:500, Invitrogen), and rinsed in $1 \times$ PBS. DAPI (4,6-diamidino-2-phenylindole) was used to counterstain. See Supplementary Table 1 for dilutions and clones. An Axio Scan.Z1 (Zeiss) and HALO (Perkin Elmer) were used for quantification. 'Positive' cells were determined by creating a threshold for fluorescence intensity, therefore, a 'negative' cell could result from an absence of the target protein or low levels of the target protein below the threshold.

In vivo vascular permeability assay and quantification. Mice were injected i.v. with $70 \mathrm{kDa}$ dextran conjugated to Texas Red (Life Technologies molecular probes) at $100 \mathrm{mg} \mathrm{kg}^{-1}$. After $3 \mathrm{~h}$, mice were injected i.v. with Lycopersicon esculentum (tomato) lectin conjugated to Dylight 488 (Vector Laboratories) at $10 \mathrm{mg} \mathrm{kg}^{-1}$. Ten minutes later, mice were euthanized by cervical dislocation ${ }^{74}$. Lungs were perfused with 4\% PFA, harvested, sectioned and imaged using an Axio Scan.Z1 (Zeiss). HALO (Perkin Elmer) was used for analysis. Perfusable vascular density was quantified as lectin-Dylight $488^{+}$cells as a percentage of total $\mathrm{DAPI}^{+}$cells in the lung. Vascular permeability was quantified as the percentage of total dextran-Texas Red fluorescence minus dextran-Texas Red colocalized with lectin-Dylight 488.

CellROX flow cytometry. Lungs were isolated, mechanically dissociated and filtered through a $40 \mu \mathrm{m}$ mesh. Cells were counted and incubated with the CellROX reagent in the dark $\left(1 \mathrm{~h}, 37^{\circ} \mathrm{C}\right.$; ThermoFisher $)$, rinsed with $1 \times \mathrm{PBS}$ and incubated with Fc block ( $1 \mathrm{~h}, 1: 100$ per $10^{6}$ cells; BD Biosciences), followed by incubation with antibodies ( $1 \mathrm{~h}$, Supplementary Table 1). A BD LSRFortessa was used for flow cytometry. OneComp eBeads (eBiosceince) were used for compensation. Dead cells and debris were excluded from analyses using forward scatter $\times$ side scatter and dead cell exclusion via live/dead stain (Invitrogen) or DAPI. Mouse neutrophils were defined as $\mathrm{CD} 45^{+} \mathrm{CD} 11 \mathrm{~b}^{+} \mathrm{Ly} 6 \mathrm{C}^{\text {lo }} \mathrm{Ly}_{6 \mathrm{G}^{+}}$, monocytes as $\mathrm{CD} 45^{+}$

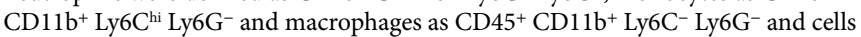
producing ROS were positive for CellROX. FlowJo was used for analysis.

Isolation of primary neutrophils and preparation of conditioned media. Cells were obtained from bone marrow (femurs and tibiae flushed) or lung (mechanical dissociation) under sterile conditions, and filtered through a $40 \mu \mathrm{m}$ mesh.
Primary neutrophils were enriched using a Neutrophil Isolation Kit (Millipore), an OctoMACs Separator column (Millipore) and MS columns (Millipore). Neutrophils were seeded into low-attachment plates in serum-free DMEM media with $1 \% \mathrm{P} / \mathrm{S}$ at 50,000 cells per $\mathrm{mm}^{2}$ at $37^{\circ} \mathrm{C} / 5 \% \mathrm{CO}_{2}$, and conditioned medium was collected over a period of $20 \mathrm{~h}$. Catalase $\left(60 \mathrm{U} \mathrm{ml}^{-1}\right)$ or methionine $(1 \mathrm{mM})$ was added during this 20 -h incubation period, unless stated otherwise.

ROS-related quantification and enzyme activity assays. Enzyme activity assays were performed on serum, or primary neutrophils from lung or bone marrow. Neutrophil and serum isolation were performed as described above. Neutrophils were sonicated and protein concentrations were determined using Pierce BCA Protein Assay Kit (Thermo). Catalase activity was quantified using a Catalase Colorimetric Activity Kit (Arbor Assays), iNOS activity was quantified using a colorimetric Nitric Oxide Synthase Activity Assay Kit (Abcam), superoxide dismutase assay (SOD) activity was quantified using a SOD Colorimetric Activity Kit (Arbor Assays), MPO activity was quantified using a colorimetric MPO Activity Assay Kit (Abcam). Hypochlorite $\left(\mathrm{ClO}^{-}\right.$, prononated form is $\left.\mathrm{HOCl}\right)$ concentration was quantified using a colorimetric Hypochlorite Assay Kit (Abcam) and nitrotyrosine (a product of tyrosine nitration by reactive nitrogen species) concentration was calculated using a nitrotyrosine enzyme-linked immunosorbent assay (ELISA) kit (Abcam). All experiments were performed according to the manufacturer's instructions and data were normalized to total protein.

Quantification of NETs. Bone-marrow neutrophils were isolated as described above and NET assays were conducted as previously described ${ }^{30,75,76}$. To quantify baseline NETosis ex vivo ${ }^{75}$, primary neutrophils were cultured in a 24 -well plate in serum-free DMEM media for $24 \mathrm{~h}$ at $37^{\circ} \mathrm{C}$ and $5 \% \mathrm{CO}_{2}$. After $24 \mathrm{~h}$, media was aspirated and NETs were washed by pipetting with PBS. This NET-containing solution was centrifuged for $10 \mathrm{~min}$ at $450 \mathrm{~g}$ at $4^{\circ} \mathrm{C}$, transferred to a $1.5 \mathrm{ml}$ tube and spun for $10 \mathrm{~min}$ at $18,000 \mathrm{~g}$ at $4^{\circ} \mathrm{C}$ to pellet the DNA. DNA was resuspended in cold $1 \times$ PBS and measured via Nanodrop. To quantify NETs in nCM, an MPO and double-stranded DNA double-target ELISA was performed as previously described ${ }^{77}$. To quantify circulating NETs, serum was collected from lean and obese mice as described above and a citrullinated histone-H3 (H3cit, clone 11D3) ELISA was performed (Cayman Chemical). To quantify NETs in lungs, immunofluorescence was performed on fixed-frozen lung tissues from obese and lean mice, using antibodies against MPO and H3cit. NETs were identified as being dually positive for both MPO and $\mathrm{H} 3$ cit, as previously described ${ }^{78}$.

Quantification of CellTracker fluorescence intensity. Cancer cells were labeled with green CellTracker dye before tail vein injection, which dilutes as cells proliferate. HALO (Perkin Elmer) was used to bin cells into three equally distributed fluorescence intensity ranges-low, medium and high - where high intensity represents the lowest proliferation category (that is, dye is retained within the cell and therefore fluorescence is brightest). For each mouse, the number of tumor cells within each bin were calculated as a percentage of total CellTracker ${ }^{+}$cells.

TEM assays. HUVECs or HMECs were seeded in transwell chambers $(8 \mu \mathrm{M}$, 24 -well format) at a density of 100,000 cells per well. Once a tight barrier was formed (confirmed by TEER), serum gradients were established between the lower and upper chambers as indicated in the figures. Py230, E0771 or MDA-MB-231 BC cells were labeled with Vybrant CFDA SE Cell Tracer (Invitrogen) to distinguish between cancer and endothelial cells. Relevant pretreatments were applied for $24 \mathrm{~h}$ before combining cells in coculture. BC cells were seeded at 4,000 cells per well in the upper chamber, and migrated to the bottom of the transwell over $24 \mathrm{~h}$. Cancer cells on the underside of the transwell chamber were quantified manually within nine fields of view using a fluorescent microscope and $\times 10$ objective.

In vitro assessment of endothelial barrier integrity. Two approaches were used to assess vascular permeability and junctional integrity in vitro, as recommended ${ }^{79}$.

TEER assays. HMECs were seeded in transwell chambers $(8 \mu \mathrm{M}, 24$-well format) at 100,000 cells per well. TEER was measured every $24 \mathrm{~h}$ using a EVOM2 Epithelial Voltmeter (World Precision Instruments) according to the manufacturer's instructions, which uses the voltage and current to quantify electrical resistance.

In vitro dextran-FITC permeability assay. An In Vitro Vascular Permeabilty Assay kit (Millipore) was used as per the manufacturer's instructions. Briefly, HUVECs were seeded in a 96-well permeability insert assembly, collagen-coated plate at 5,000 cells per insert. Once a tight barrier was formed, treatments were administered for $24 \mathrm{~h}$ as indicated in the figures. Dextran-FITC was then added for $10 \mathrm{~min}$ at the recommended dilution (1:40). Fluorescence that leaked through the insert was quantified using a plate reader at $485 \mathrm{~nm}$ excitation and $535 \mathrm{~nm}$ emission.

Subcellular fractionation assay. HUVECs were seeded in a $10 \mathrm{~cm}$ plate and allowed to grow to confluence. Confluent HUVECs were treated with Medium 199 with $5 \%$ mouse serum for $24 \mathrm{~h}$. Cell pellets were gathered by cell scraping and subcellular fractionation was performed using a Subcellular Protein Fractionation 
kit (ThermoFisher Scientific). Protein concentration was determined using a Pierce BCA Protein Assay Kit (Thermo) followed by western blot.

Western blot analysis on whole-lung tissue and cell lysates. Lysates from tissues were obtained via bead shaker homogenization (BD), or from cells via scraping in RIPA buffer (Thermo). Protein concentrations were determined using a Pierce BCA Protein Assay Kit (Thermo). Samples were loaded onto a 4-12\% Bis-Tris-PAGE gel (Life Technologies, $10 \mu \mathrm{g}$ of protein per lane). Proteins were transferred to polyvinylidene difluoride membranes for $1 \mathrm{~h}$ at $4{ }^{\circ} \mathrm{C}$. Membranes were blocked using $5 \%$ milk in Tris-buffered saline with $0.05 \%$ Tween (TBS-T) for $1 \mathrm{~h}$, and incubated with primary antibody overnight at $4{ }^{\circ} \mathrm{C}$ with shaking (Supplementary Table 1). Membranes were rinsed in TBS-T and secondary horseradish peroxidase-conjugated antibodies were applied for $1 \mathrm{~h}$ at room temperature with shaking. Membranes were rinsed with TBS-T, and bands were visualized using enhanced chemiluminescence reagent (Bio-Rad) and an Amersham Imager 600 (GE Healthcare Life Sciences). If needed, stripping buffer (ThermoFisher Scientific) was applied to the membrane for $15 \mathrm{~min}$ at room temperature with shaking. Bands were analyzed by densitometry using Amersham 600 Analysis Software. Protein quantification was normalized to $\beta$-actin or control bands as indicated.

shRNA knockdown. HEK293T Lenti-X cells (from ATCC) were transfected with plasmids using Lipo2000 Transfection Reagent (ThermoFisher Scientific). Viral supernatant was collected after $24 \mathrm{~h}$, filtered through a $0.45-\mu \mathrm{m}$ syringe filter and added to target cells (seeded at 500,000 cells per well $24 \mathrm{~h}$ before). This was repeated $24 \mathrm{~h}$ later. Cells were rinsed, and selection media containing $2.5 \mu \mathrm{g} \mathrm{ml}^{-1}$ puromycin was applied (Wisent Bio Products) for a minimum of $7 \mathrm{~d}$ to ensure successful selection, as confirmed by western blot. All shRNA constructs were obtained from the High Throughput Screening Facility (Life Science Complex, McGill University):

(1) Jam1 shRNA no. 1 in HMECs,F11R clone no. R-707-3, TRC Clone ID TRCN0000061649, Gene ID 50848,CCGGCCAGACTCGTTTGCTATAATACTCGAGTATTATAGCAAACGAGTCTGGTTTTTG

(2) Jam 1 shRNA no. 2 in HMECs,F11R clone no. R-896-2, TRC Clone ID TRCN0000061652, Gene ID 50848,CCGGGCCAACTGGTATCACCTTCAACTCGAGTTGAAGGTGATACCAGTTGGCTTTTTG

(3) Jam 1 shRNA in Py230 cells,F11R clone no. R-896-M-6, TRC Clone ID TRCN00000271840, Gene ID 16456,CCGGCCTGGTTCAAGGACGGGATATCTCGAGATATCCCGTCCTTGAACCAGGTTTTTG

(4) Vcam1 shRNA in HMECs,VCAM1 clone no. R-874-10, TRC Clone ID TRCN0000123172, Gene ID 7412,CCGGCCAGATAGATAGTCCACTGAACTCGAGTTCAGTGGACTATCTATCTGGTTTTTG

(5) Cat-shRNA in HMECs, CAT clone no. R-874-6, TRC Clone ID TRCN000061754, Gene ID 847, CCGGGCCACATGAATGGATATG GATCTCGAGATCCATATCCATTCATGTGGCTTTTTG

Mouse XL cytokine array. Lung $\mathrm{nCM}$ was obtained from LF or HF mice as described above, and 111 soluble proteins were quantified using a Mouse XL Cytokine Array Kit (R\&D Systems). Protein detection was visualized using a Chemi Reagent Mix (R\&D Systems) and an Amersham Imager 600 chemidoc (GE Healthcare Life Sciences). Pixel density was quantified by densiometric analysis using the Amersham 600 Analysis Software.

RNA-seq. Neutrophils $\left(\mathrm{CD} 45^{+} \mathrm{CD} 11 \mathrm{~b}^{+} \mathrm{Ly}_{6 \mathrm{C}^{\mathrm{lo}}} \mathrm{Ly}_{6 \mathrm{G}}{ }^{+}\right)$or endothelial cells $\left(\mathrm{CD} 45^{-} \mathrm{CD} 31^{+}\right)$were FACS-purified from lungs of LF or HF mice. RNA-seq and quality control were performed at GENEWIZ. RNA was isolated with TRIzol LS (Invitrogen) and integrity was assessed via Agilent Bioanalyzer 2100. A SMART-Seq library preparation kit was used, and $2 \times 100$ base-pair sequencing was performed on an Illumina HISeq 2000. Fastp (v.0.20.0) was used to collect quality control metrics of the raw reads. RNA sequences were aligned and sorted by coordinates, to the National Center for Biotechnology Information mouse genome build 38 v.96, using STAR aligner (STAR-2.6.1b). The removal of alignment duplicates was done with Sambamba (v.0.7.0). Quantification of genes was performed using featureCounts (v.2.0.0). DESeq2 (v.1.24.0) was used to normalize feature counts and to test for differentially expressed genes. The HGNC symbols were extracted and added to the DESeq 2 results data frame using biomaRt (v.2.40.4) using the 'mmusculus_gene_ensembl' dataset and the Ensembl Release 96 (April 2019).

RNA-seq pathway analysis. Pathway enrichment in lung neutrophils was assessed using IPA software v.01-13 (Qiagen). The top 1,000 differentially expressed genes $(P<0.001 \pm 2$ fold change) was selected and 'Core Analysis' was used with all default parameters. RNA-seq normalized read counts from lung neutrophils were converted to.gmt format and analyzed using GSEA software v.4.1.0 (Broad Institute). Analysis was completed using default settings. Gene set database, 'h.all. v6.2.symbols.gmt'. Chip platform, 'Mouse Gene Symbol Remapping Human Orthologs MSigDB.v7.2.chip. Transcription factor analysis on lung endothelial cells was performed using DoRothEA v.1.3.0, a gene set resource containing signed transcription factor-target interactions ${ }^{12}$. We conducted all the analyses in RStudio v.3.6.1.

IMC. Antibodies were optimized via immunofluorescence and validated by clinical pathologist, M.-C. Guiot. Conjugations were carried out by the Single Cell and Imaging Mass Cytometry Platform at the Goodman Cancer Research Centre (McGill University), using Maxpar Conjugation Kits (Fluidigm). The protocol for human sample biobanking was approved by the Montreal University Health Centre Research Ethics Board, no. 2009-5354. All samples were collected with informed consent from patients. Formalin-fixed paraffin-embedded (FFPE) lung metastasis samples were obtained from P. Fiset (pathologist) and J. Spicer (thoracic surgeon), and cores within tumor centers were selected by K. Lach and M. Issac (pathology) to generate a tissue microarray. BMI values of patients ranged from 19.9 to 36.3 and ages ranged from 46 to 86 . Deparaffinization and heat-induced epitope retrieval were performed using the Ventana Discovery Ultra auto-stainer platform (Roche Diagnostics). FFPE slides were incubated in EZ Prep solution (preformulated, Roche Diagnostics) at $70^{\circ} \mathrm{C}$ to deparaffinize, followed by antigen-retrieval in standard Cell Conditioning 1 solution (CC1, preformulated; Roche Diagnostics) at $95^{\circ} \mathrm{C}$. Slides were then washed in $1 \times$ PBS, blocked in Dako Serum-free Protein Block solution (Agilent), followed by antibody staining overnight at $4{ }^{\circ} \mathrm{C}$ as described by Fluidigm for FFPE tissues. Tissues were stained with a panel of 35 multiplexed metal-conjugated antibodies (Extended Data Fig. 8a,b). IMC images were acquired at a resolution of roughly $1 \mu \mathrm{m}$, frequency of $200 \mathrm{~Hz}$ and area of $1 \mathrm{~mm}^{2}$, Hyperion Imaging System and CyTOF Software v.6.7.1014 (Fluidigm).

IMC analysis. Cell segmentation, intensity calculations, cell assignment and $t$-SNE graphs were generated using a custom computational pipeline in MATLAB v.7.10. Briefly, foreground and background staining for each marker was modeled as a mixture of two Gaussians distributions. Cell segmentation was achieved by assessing the gradient magnitude, seed contour and scale space for each nuclei, followed by Chan-Vese ${ }^{80}$. Basic cell lineage assignments were defined by the following markers: cancer, $\mathrm{PanCK}^{+}$; macrophages, $\mathrm{CD}^{+} 8^{+}$; neutrophils, $\mathrm{MPO}^{+}$; endothelial cells, CD $31^{+}$; B cells, CD20 ${ }^{+}$; cytotoxic T cells, $\mathrm{CD}^{+}{ }^{+} \mathrm{CD} 8^{+}$and helper $\mathrm{T}$ cells, $\mathrm{CD}^{+} \mathrm{CD}^{+}$. MCD Viewer software was used to generate representative images (Fluidigm). Patients with a BMI $<25.9$ were assigned to the $\mathrm{BMI}^{\text {low }}$ group (average BMI, 24 and average age, 76), and patients with a BMI $>26$ were assigned to the $\mathrm{BMI}^{\text {high }}$ group (average BMI of 30 and average age of 70 ).

Statistics and reproducibility. GraphPad Prism Pro v.7 or v.8 were used for data analysis. Data are presented as mean \pm s.e.m. unless indicated otherwise, and $P<0.05$ was considered statistically significant. For all representative images, results were reproduced at least three times in independent experiments. For all quantitative data, the statistical test used is indicated in the legends. A statistics 'decision tree' is included in Supplementary Fig. 1, along with all raw data used for statistics. Briefly, normal distribution was first determined using the Shapiro-Wilk normality test. Data determined to be parametric were analyzed by an unpaired Student's $t$-test (two groups), or ordinary one-way analysis of variance (ANOVA) ( $>2$ groups) with Bonferroni's multiple comparisons test for select pairwise comparisons as indicated. Data determined to be nonparametric were analyzed by a Mann-Whitney test (two groups), or Kruskal-Wallis test ( $>2$ groups) with Dunn's multiple comparisons for select pairwise comparisons as indicated. All tests were two-tailed unless otherwise indicated. For patient tissue analysis, Pearson's correlation was used to assess relationship with BMI. All data included in the study are reproducible: all western blots were performed three or more times with similar results; all flow cytometry experiments were repeated three or more times with similar results; all animal trials were repeated with at least $n=4$ in at least two independent cohorts with similar results; all in vitro assays were repeated in at least three independent experiments with similar results and the $n$-value within each figure legend represents the number of experimental replicates. Sample sizes were chosen based on power of 0.9 to detect a difference of $>1.5$ standard deviations between means with $95 \%$ confidence. However, in most cases, previous data were sufficient to inform sample size for subsequent experiments. Pre-established exclusion criteria included mice on a LF diet $>28 \mathrm{~g}$ and mice on a HF diet $<28 \mathrm{~g}$, as this would be considered atypical and rare for the DIO model. When comparing treatment efficacy in lean and obese animals, allocation of mice to specific treatment groups ensured similar distribution of body weight. For all other experiments, no specific randomization method was followed. No specific blinding method was followed, however, automated quantitative methods were used to eliminate subjective interpretation of data.

Reporting Summary. Further information on research design is available in the Nature Research Reporting Summary linked to this article.

\section{Data availability}

Numerical and western blot source data for Figs. 1-8 and Extended Data Figs. 1-8 are provided with this paper. Any additional data that support the findings of this study are available from the corresponding author upon reasonable request. Further information on research design is available in the Nature Research Reporting Summary linked to this article. All RNA-seq data were deposited 
in the Gene Expression Omnibus under the accession codes GSE165442 (lung neutrophils, Figs. 4a-d and 6c and Extended Data Fig. 6f) and GSE165441 (lung endothelial cells, Fig. 2a-c). Databases used for RNA-seq analysis included MSigDB v.7.2 http://www.gsea-msigdb.org/gsea/msigdb/index.jsp, DoRothEA v.1.3.0 https://saezlab.github.io/dorothea/ and IPA v.01-13 https://digitalinsights. qiagen.com.

\section{Code availability}

MATLAB code used for IMC cell segmentation is available upon request.

Received: 14 October 2019; Accepted: 10 March 2021; Published online: 3 May 2021

\section{References}

1. Lauby-Secretan, B. et al. Body fatness and cancer-viewpoint of the IARC working group. N. Engl. J. Med. 375, 794-798 (2016).

2. Calle, E. E., Rodriguez, C., Walker-Thurmond, K. \& Thun, M. J. Overweight, obesity, and mortality from cancer in a prospectively studied cohort of U.S. adults. N. Engl. J. Med. 348, 1625-1638 (2003).

3. Risk, N. C. D. Factor collaboration. Trends in adult body-mass index in 200 countries from 1975 to 2014: a pooled analysis of 1698 populationbased measurement studies with 19.2 million participants. Lancet $\mathbf{3 8 7}$ 1377-1396 (2016)

4. Calle, E. E. \& Kaaks, R. Overweight, obesity and cancer: epidemiological evidence and proposed mechanisms. Nat. Rev. Cancer 4, 579-591 (2004).

5. Ligibel, J. A. et al. American Society of Clinical Oncology position statement on obesity and cancer. J. Clin. Oncol. 32, 3568-3574 (2014).

6. Weigelt, B., Peterse, J. L. \& van 't Veer, L. J. Breast cancer metastasis: markers and models. Nat. Rev. Cancer 5, 591-602 (2005).

7. Ewertz, M. et al. Effect of obesity on prognosis after early-stage breast cancer. J. Clin. Oncol. 29, 25-31 (2011).

8. Osman, M. A. \& Hennessy, B. T. Obesity correlation with metastases development and response to first-line metastatic chemotherapy in breast cancer. Clin. Med. Insights Oncol. 9, 105-112 (2015).

9. Olson, O. C., Quail, D. F. \& Joyce, J. A. Obesity and the tumor microenvironment. Science 358, 1130-1131 (2017).

10. Quail, D. F. et al. Obesity alters the lung myeloid cell landscape to enhance breast cancer metastasis through IL5 and GM-CSF. Nat. Cell Biol. 19 974-987 (2017).

11. Adams, T. D. et al. Long-term mortality after gastric bypass surgery. N. Engl. J. Med. 357, 753-761 (2007).

12. Garcia-Alonso, L., Holland, C. H., Ibrahim, M. M., Turei, D. \& Saez-Rodriguez, J. Benchmark and integration of resources for the estimation of human transcription factor activities. Genome Res. 29, 1363-1375 (2019).

13. Czymai, T. et al. FOXO3 modulates endothelial gene expression and function by classical and alternative mechanisms. J. Biol. Chem. 285, 10163-10178 (2010).

14. Potente, M. et al. Involvement of Foxo transcription factors in angiogenesis and postnatal neovascularization. J. Clin. Invest. 115, 2382-2392 (2005).

15. Sang, T., Cao, Q., Wang, Y., Liu, F. \& Chen, S. Overexpression or silencing of FOXO3a affects proliferation of endothelial progenitor cells and expression of cell cycle regulatory proteins. PLOS ONE 9, e101703 (2014).

16. Weber, C., Fraemohs, L. \& Dejana, E. The role of junctional adhesion molecules in vascular inflammation. Nat. Rev. Immunol. 7, 467-477 (2007).

17. Phillipson, M. \& Kubes, P. The neutrophil in vascular inflammation. Nat. Med. 17, 1381-1390 (2011).

18. Boivin, G. et al. Durable and controlled depletion of neutrophils in mice. Nat Commun. 11, 2762 (2020).

19. Gomez-Moreno, D., Adrover, J. M. \& Hidalgo, A. Neutrophils as effectors of vascular inflammation. Eur. I. Clin. Invest. 48, el2940 (2018).

20. Rohn, T. T. et al. Priming of human neutrophils by peroxynitrite: potential role in enhancement of the local inflammatory response. J. Leukoc. Biol. $\mathbf{6 5}$, 59-70 (1999).

21. Manda-Handzlik, A. et al. Nitric oxide and peroxynitrite trigger and enhance release of neutrophil extracellular traps. Cell. Mol. Life Sci. 77, 3059-3075 (2020)

22. Yabe, Y., Nishikawa, M., Tamada, A., Takakura, Y. \& Hashida, M. Targeted delivery and improved therapeutic potential of catalase by chemical modification: combination with superoxide dismutase derivatives. $J$. Pharmacol. Exp. Ther. 289, 1176-1184 (1999).

23. Chen, A. Y., Lu, J. M., Yao, Q. \& Chen, C. Entacapone is an antioxidant more potent than vitamin $\mathrm{C}$ and vitamin $\mathrm{E}$ for scavenging of hypochlorous acid and peroxynitrite, and the inhibition of oxidative stress-induced cell death. Med. Sci. Monit. 22, 687-696 (2016).

24. Song, E. et al. Deamidated lipocalin-2 induces endothelial dysfunction and hypertension in dietary obese mice. J. Am. Heart Assoc. 3, e000837 (2014).

25. Florence, J. M., Krupa, A., Booshehri, L. M., Allen, T. C. \& Kurdowska, A. K. Metalloproteinase-9 contributes to endothelial dysfunction in atherosclerosis via protease activated receptor-1. PLoS ONE 12, e0171427 (2017).
26. Saha, P. et al. Bacterial siderophores hijack neutrophil functions. J. Immunol. 198, 4293-4303 (2017).

27. Ward, P. P., Mendoza-Meneses, M., Park, P. W. \& Conneely, O. M. Stimulus-dependent impairment of the neutrophil oxidative burst response in lactoferrin-deficient mice. Am. J. Pathol. 172, 1019-1029 (2008).

28. Metzler, K. D. et al. Myeloperoxidase is required for neutrophil extracellular trap formation: implications for innate immunity. Blood 117, 953-959 (2011).

29. Clark, S. R. et al. Platelet TLR4 activates neutrophil extracellular traps to ensnare bacteria in septic blood. Nat. Med. 13, 463-469 (2007).

30. Albrengues, J. et al. Neutrophil extracellular traps produced during inflammation awaken dormant cancer cells in mice. Science 361, eaao4227 (2018)

31. Szczerba, B. M. et al. Neutrophils escort circulating tumour cells to enable cell cycle progression. Nature 566, 553-557 (2019).

32. Perreault, M. \& Marette, A. Targeted disruption of inducible nitric oxide synthase protects against obesity-linked insulin resistance in muscle. Nat. Med. 7, 1138-1143 (2001).

33. Amano, S. U. et al. Local proliferation of macrophages contributes to obesity-associated adipose tissue inflammation. Cell Metab. 19, 162-171 (2014).

34. Kichenadasse, G. et al. Association between body mass index and overall survival with immune checkpoint inhibitor therapy for advanced non-small cell lung cancer. JAMA Oncol. 6, 512-518 (2019).

35. McQuade, J. L. et al. Association of body-mass index and outcomes in patients with metastatic melanoma treated with targeted therapy, immunotherapy, or chemotherapy: a retrospective, multicohort analysis. Lancet Oncol. 19, 310-322 (2018).

36. Domigan, N. M., Charlton, T. S., Duncan, M. W., Winterbourn, C. C. \& Kettle, A. J. Chlorination of tyrosyl residues in peptides by myeloperoxidase and human neutrophils. J. Biol. Chem. 270, 16542-16548 (1995).

37. Harris, I. S. \& DeNicola, G. M. The complex interplay between antioxidants and ROS in cancer. Trends Cell Biol. 30, 440-451 (2020).

38. Blot, W. J. et al. Nutrition intervention trials in Linxian, China: supplementation with specific vitamin/mineral combinations, cancer incidence, and disease-specific mortality in the general population. J. Natl Cancer Inst. 85, 1483-1492 (1993)

39. Keaney, J. F. Jr. et al. Obesity and systemic oxidative stress: clinical correlates of oxidative stress in the Framingham Study. Arterioscler. Thromb. Vasc. Biol. 23, 434-439 (2003).

40. Aleman, J. O. et al. Effects of rapid weight loss on systemic and adipose tissue inflammation and metabolism in obese postmenopausal women. J. Endocr. Soc. 1, 625-637 (2017).

41. Aruoma, O. I., Halliwell, B., Hoey, B. M. \& Butler, J. The antioxidant action of $\mathrm{N}$-acetylcysteine: its reaction with hydrogen peroxide, hydroxyl radical, superoxide, and hypochlorous acid. Free Radic. Biol. Med. 6, 593-597 (1989).

42. Nagareddy, P. R. et al. Adipose tissue macrophages promote myelopoiesis and monocytosis in obesity. Cell Metab. 19, 821-835 (2014)

43. Herishanu, Y., Rogowski, O., Polliack, A. \& Marilus, R. Leukocytosis in obese individuals: possible link in patients with unexplained persistent neutrophilia. Eur. J. Haematol. 76, 516-520 (2006).

44. Gentles, A. J. et al. The prognostic landscape of genes and infiltrating immune cells across human cancers. Nat. Med. 21, 938-945 (2015).

45. Coffelt, S. B., Wellenstein, M. D. \& de Visser, K. E. Neutrophils in cancer: neutral no more. Nat. Rev. Cancer 16, 431-446 (2016).

46. Kwak, H. J. et al. Myeloid cell-derived reactive oxygen species externally regulate the proliferation of myeloid progenitors in emergency granulopoiesis. Immunity 42, 159-171 (2015).

47. Adrover, J. M. et al. A neutrophil timer coordinates immune defense and vascular protection. Immunity 50, 390-402 e310 (2019).

48. Ode, Y., Aziz, M. \& Wang, P. CIRP increases ICAM-1(+) phenotype of neutrophils exhibiting elevated iNOS and NETs in sepsis. J. Leukoc. Biol. 103 693-707 (2018)

49. Bronte, V. et al. Recommendations for myeloid-derived suppressor cell nomenclature and characterization standards. Nat. Commun. 7, 12150 (2016).

50. Xia, S. et al. Gr-1+ $\mathrm{CD}^{+1} \mathrm{~b}^{+}$myeloid-derived suppressor cells suppress inflammation and promote insulin sensitivity in obesity. J. Biol. Chem. 286, 23591-23599 (2011)

51. Clements, V. K. et al. Frontline science: high fat diet and leptin promote tumor progression by inducing myeloid-derived suppressor cells. J. Leukoc. Biol. 103, 395-407 (2018).

52. Ng, L. G., Ostuni, R. \& Hidalgo, A. Heterogeneity of neutrophils. Nat. Rev. Immunol. 19, 255-265 (2019).

53. Eiserich, J. P. et al. Myeloperoxidase, a leukocyte-derived vascular NO oxidase. Science 296, 2391-2394 (2002).

54. Wang, Q. et al. Myeloperoxidase deletion prevents high-fat diet-induced obesity and insulin resistance. Diabetes 63, 4172-4185 (2014).

55. Zhou, J., Wang, Q., Ding, Y. \& Zou, M. H. Hypochlorous acid via peroxynitrite activates protein kinase Ctheta and insulin resistance in adipocytes. J. Mol. Endocrinol. 54, 25-37 (2015). 
56. Kessenbrock, K. et al. Netting neutrophils in autoimmune small-vessel vasculitis. Nat. Med. 15, 623-625 (2009).

57. Moorthy, A. N., Tan, K. B., Wang, S., Narasaraju, T. \& Chow, V. T. Effect of high-fat diet on the formation of pulmonary neutrophil extracellular traps during influenza pneumonia in BALB/c Mice. Front. Immunol. 7, 289 (2016).

58. Tohme, S. et al. Neutrophil extracellular traps promote the development and progression of liver metastases after surgical stress. Cancer Res. 76, 1367-1380 (2016).

59. Cools-Lartigue, J. et al. Neutrophil extracellular traps sequester circulating tumor cells and promote metastasis. J. Clin. Invest. 123, 3446-3458 (2013)

60. Brotfain, E. et al. Neutrophil functions in morbidly obese subjects. Clin. Exp. Immunol. 181, 156-163 (2015).

61. Shah, D. et al. Obesity-induced adipokine imbalance impairs mouse pulmonary vascular endothelial function and primes the lung for injury. Sci. Rep. 5, 11362 (2015).

62. Chedid, P. et al. Adiponectin and its globular fragment differentially modulate the oxidative burst of primary human phagocytes. Am. J. Pathol. 180, 682-692 (2012).

63. Tilg, H. \& Moschen, A. R. Adipocytokines: mediators linking adipose tissue, inflammation and immunity. Nat. Rev. Immunol. 6, 772-783 (2006)

64. Miyazaki, Y. et al. Effect of pioglitazone on circulating adipocytokine levels and insulin sensitivity in type 2 diabetic patients. J. Clin. Endocrinol. Metab. 89, 4312-4319 (2004).

65. Quail, D. F. \& Dannenberg, A. J. The obese adipose tissue microenvironment in cancer development and progression. Nat. Rev. Endocrinol. 15, 139-154 (2019).

66. Guy, C. T., Cardiff, R. D. \& Muller, W. J. Induction of mammary tumors by expression of polyomavirus middle $\mathrm{T}$ oncogene: a transgenic mouse model for metastatic disease. Mol. Cell. Biol. 12, 954-961 (1992).

67. Gibby, K. et al. Early vascular deficits are correlated with delayed mammary tumorigenesis in the MMTV-PyMT transgenic mouse following genetic ablation of the NG2 proteoglycan. Breast Cancer Res. 14, R67 (2012).

68. Ewens, A., Mihich, E. \& Ehrke, M. J. Distant metastasis from subcutaneously grown E0771 medullary breast adenocarcinoma. Anticancer Res. 25, 3905-3915 (2005).

69. Nishikawa, M. et al. Inhibition of metastatic tumor growth by targeted delivery of antioxidant enzymes. J. Control. Release 109, 101-107 (2005).

70. Kaushik, N. et al. Responses of solid tumor cells in DMEM to reactive oxygen species generated by non-thermal plasma and chemically induced ROS systems. Sci. Rep. 5, 8587 (2015).

71. Avci, B., Bahadir, A., Tuncel, O. K. \& Bilgici, B. Influence of alpha-tocopherol and alpha-lipoic acid on bisphenol-A-induced oxidative damage in liver and ovarian tissue of rats. Toxicol. Ind. Health 32, 1381-1390 (2016).

72. Davie, S. A. et al. Effects of $\mathrm{FVB} / \mathrm{NJ}$ and $\mathrm{C} 57 \mathrm{Bl} / 6 \mathrm{~J}$ strain backgrounds on mammary tumor phenotype in inducible nitric oxide synthase deficient mice. Transgenic Res. 16, 193-201 (2007)

73. Quail, D. F. et al. The tumor microenvironment underlies acquired resistance to CSF-1R inhibition in gliomas. Science 352, aad3018 (2016).

74. Huang, Y. et al. Pulmonary vascular destabilization in the premetastatic phase facilitates lung metastasis. Cancer Res. 69, 7529-7537 (2009).

75. Najmeh, S., Cools-Lartigue, J., Giannias, B., Spicer, J. \& Ferri, L. E. Simplified human neutrophil extracellular traps (NETs) isolation and handling. J. Vis. Exp. 16, 52687 (2015).

76. Rayes, R. F. et al. Primary tumors induce neutrophil extracellular traps with targetable metastasis promoting effects. JCI Insight 5, e128008 (2019).

77. Yoo, D. G., Floyd, M., Winn, M., Moskowitz, S. M. \& Rada, B. NET formation induced by Pseudomonas aeruginosa cystic fibrosis isolates measured as release of myeloperoxidase-DNA and neutrophil elastase-DNA complexes. Immunol. Lett. 160, 186-194 (2014).
78. Park, J. et al. Cancer cells induce metastasis-supporting neutrophil extracellular DNA traps. Sci. Transl. Med. 8, 361 ra138 (2016).

79. Robinson, B. D., Shaji, C. A., Lomas, A. \& Tharakan, B. Measurement of microvascular endothelial barrier dysfunction and hyperpermeability in vitro. Methods Mol. Biol. 1717, 237-242 (2018)

80. Chan, T. F. \& Vese, L. A. Active contours without edges. IEEE Trans. Image Process. 10, 266-277 (2001).

\section{Acknowledgements}

We thank the Goodman Cancer Research Centre and Life Sciences Complex at McGill University for core facility support, including the Single Cell and Imaging Mass Cytometry Platform (SCIMAP, Y.W.), Histology facility (Cleber Moraes), the Comparative Medicine and Animal Resource Centre and the Flow Cytometry facility. SCIMAP is supported by a McGill MI4 Platform grant and the Fraser Memorial Trust. This study was funded by the Susan G. Komen Foundation (D.F.Q., grant no. CCR18548032), Canada Foundation for Innovation (D.F.Q., JELF-37488; L.A.W., JELF-39178), Canadian Institutes of Health Research (D.F.Q., PJT-159742; L.A.W., PJT162137) and the Breast Cancer Research Foundation (A.J.D., BCRF-19-034). D.F.Q. is supported by a Tier II Canada Research Chair in Tumor Microenvironment Research. L.A.W. is supported by a Rosalind and Morris Goodman Chair in Lung Cancer Research. S.A.C.M is supported by a Charlotte and Leo Karassik Foundation Fellowship, a Canada Graduate Scholarship from the Canadian Institutes of Health Research and a Canderel Graduate Studentship Award from the Goodman Cancer Research Centre.

\section{Author contributions}

S.A.C.M., L.A.W. and D.F.Q. designed the study, reviewed the data and wrote the paper. A.J.D. provided essential insight throughout the study and contributed neutrophil RNA-seq data. S.A.C.M. oversaw and performed all experiments and data analysis. R.B.E.L., E.K., M.R., R.R.Y. and B.F. provided bioinformatics support. R.B.E.L., A.A., S.D., N.C.B., V.B., B.S., R.F.R. and L.K. provided experimental support and/or optimized protocols. L.J.M.P., D.M., Y.W. and I.R.W. optimized the IMC antibody panel. K.D.L., M.S.M.I., R.F.R., M-C.G., P.O.F. and J.D.S. provided human tissue samples, built the patient tissue microarray and provided pathology/clinical insight. All authors reviewed and approved the paper.

\section{Competing interests}

P.O.F. has received honoraria and consultancy fees from Amgen Canada, AstraZeneca Canada, Bristol Myers Squibb, EMD Serono, Hoffmann-La Roche, Merck Canada, Pfizer Canada and Roche Canada. J.D.S. has received consultancy fees for BMS, Merck, Amgen, AstraZeneca, Protalix and TransHit Bio, and grant funding from AstraZeneca, CLS Therapeutics, Merck and Hoffman La Roche. All other authors declare no competing interests.

\section{Additional information}

Extended data is available for this paper at https://doi.org/10.1038/s43018-021-00194-9. Supplementary information The online version contains supplementary material available at https://doi.org/10.1038/s43018-021-00194-9.

Correspondence and requests for materials should be addressed to L.A.W. or D.F.Q.

Peer review information Nature Cancer thanks Luisa Iruela-Arispe, Elena Piskounova and the other, anonymous, reviewer(s) for their contribution to the peer review of this work.

Reprints and permissions information is available at www.nature.com/reprints. Publisher's note Springer Nature remains neutral with regard to jurisdictional claims in published maps and institutional affiliations.

(c) The Author(s), under exclusive licence to Springer Nature America, Inc. 202 

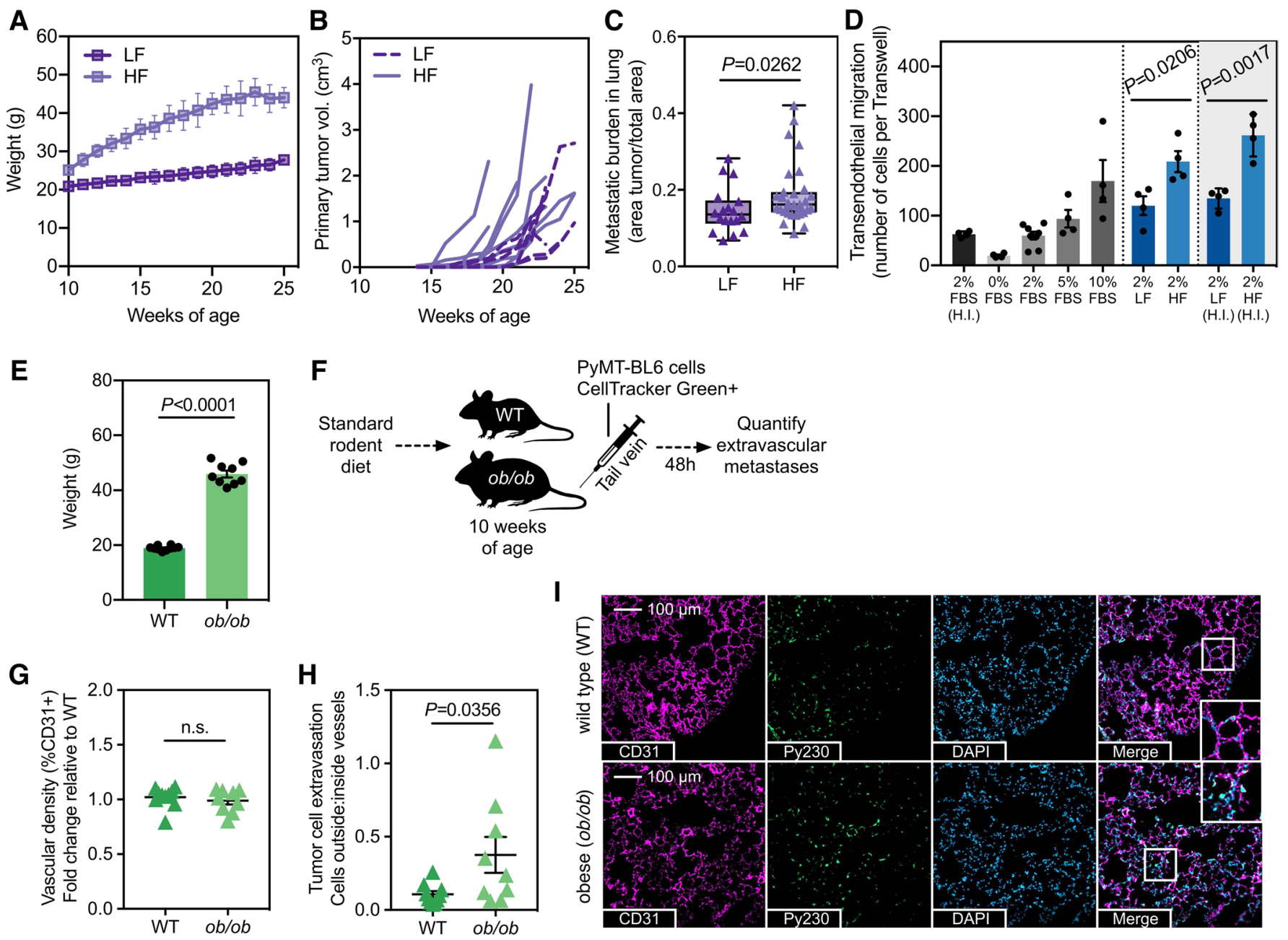

Extended Data Fig. 1 | Obesity is associated with enhanced lung metastasis in multiple genetic models. a, Weight curves for C57BL6 MMTV-PyMT mice enrolled on LF or HF diet from 5-25 weeks of age. LF, $n=7$ mice; HF, $n=9$ mice; mean \pm SEM. $\mathbf{b}$, Spider-plot showing primary tumor growth for trial shown in a, measured by digital caliper. c, Quantification of metastatic burden in lung from MMTV-PyMT mice at end point. LF, $n=18$ lung sections; HF, $\mathrm{n}=31$ lung sections; $\mathrm{Box}=$ median \pm interquartile range, whiskers = min-max, all datapoints shown; two-tailed Mann-Whitney test. $\mathbf{d}$, Control conditions for TEM assays using serum gradients. Upper chamber of the Transwell contains $0 \%$ serum in all cases. Bottom conditions include: $2 \%$ heat-inactivated (H.I.) FBS ( $n=4$ Transwells), a dose-response from 0\%-10\% FBS (2\%, $n=8$ Transwells, $0,5,10 \%, n=4$ Transwells), $2 \%$ serum isolated from LF or HF mice ( $n=4$ Transwells), and $2 \%$ serum isolated from LF or HF and subject to H.I. ( $n=4$ Transwells); mean \pm SEM; two-tailed Student's t-test. Transwells represent individual experimental replicates with similar results; mouse serum was obtained from different mice. e, Average weight of the leptin-deficient model of genetic-induced obesity (GIO). Wild type (WT) or B6.Cg-Lepob (ob/ob) mice on a C57BL6 background were raised on a standard rodent diet until 10 weeks of age. WT, $\mathrm{n}=10$ mice; ob/ob, $\mathrm{n}=9$ mice; mean $\pm \mathrm{SEM}$; two-tailed Student's t-test. $\mathbf{f}$, Schematic illustration of the in vivo extravasation assay corresponding to $\mathrm{g}$-i. WT or ob/ob mice were injected via tail vein with syngeneic breast cancer cells labelled with a green fluorescent CellTracker, and extravasation was quantified in lung after $48 \mathrm{~h}$ via fluorescence microscopy. $\mathbf{g}$, Quantification of lung vascular density in the in vivo extravasation assay. Vascular density is quantified as CD31+ cells as a percentage of total DAPI+ cells. WT, $\mathrm{n}=10$ mice; ob/ob, $\mathrm{n}=9$ mice; mean \pm SEM; two-tailed Student's t-test. $\mathbf{h}$, Fluorescence quantification of Py230 cancer cell extravasation in lung tissue. Extravasation is quantified as the ratio of cancer cells outside the vessels (CellTracker ${ }^{+}$CD31) versus inside the vessels (CellTracker ${ }^{+}$CD31+). WT, $\mathrm{n}=10$ mice; ob/ob, $\mathrm{n}=9$ mice; mean \pm SEM; two-tailed Student's t-test. i, Representative immunofluorescence image for data in $\mathrm{h}$. 
A

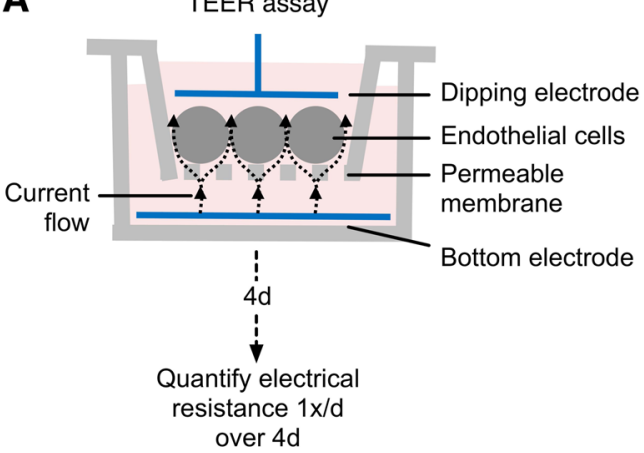

B in vitro dextran-FITC

permeability assay

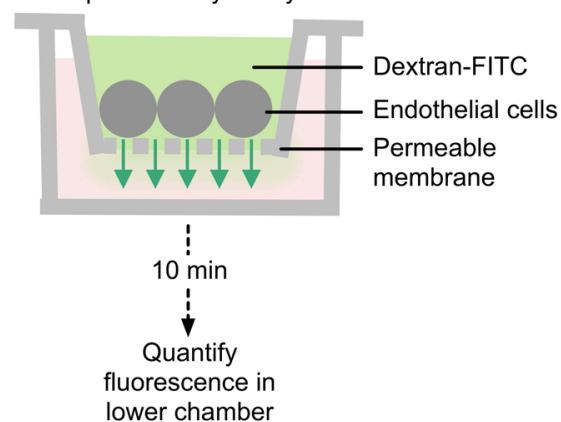

C

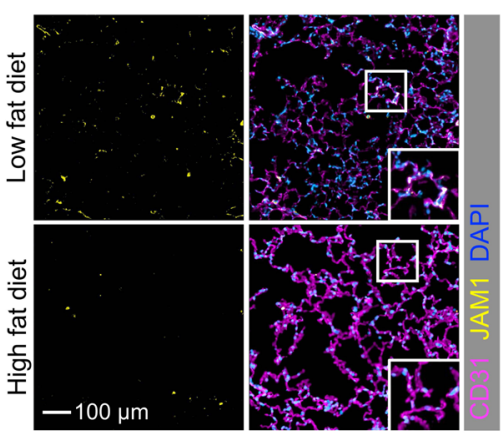

D
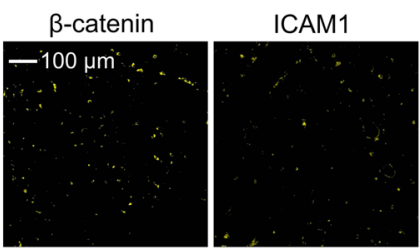

$\mathrm{N}$-cadherin
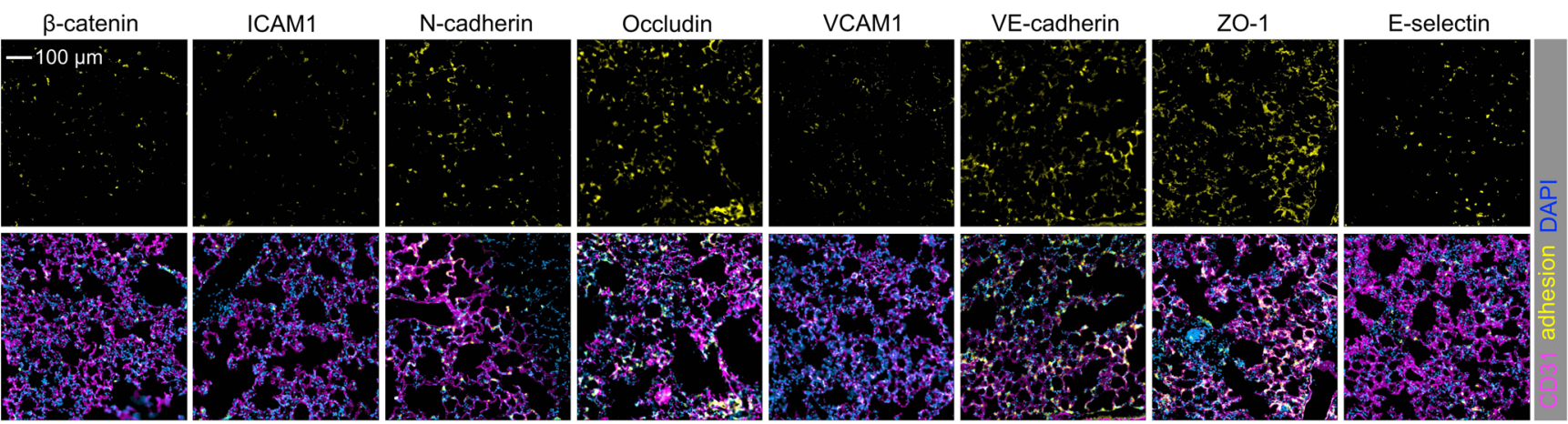

E

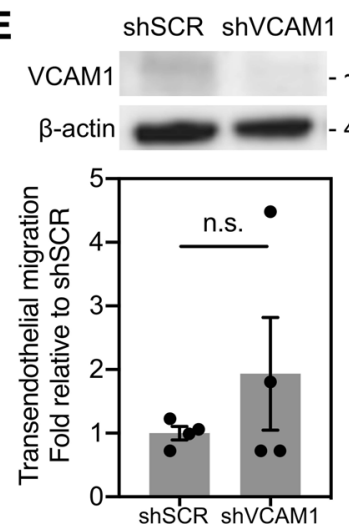

F

ShRNA \#1

G
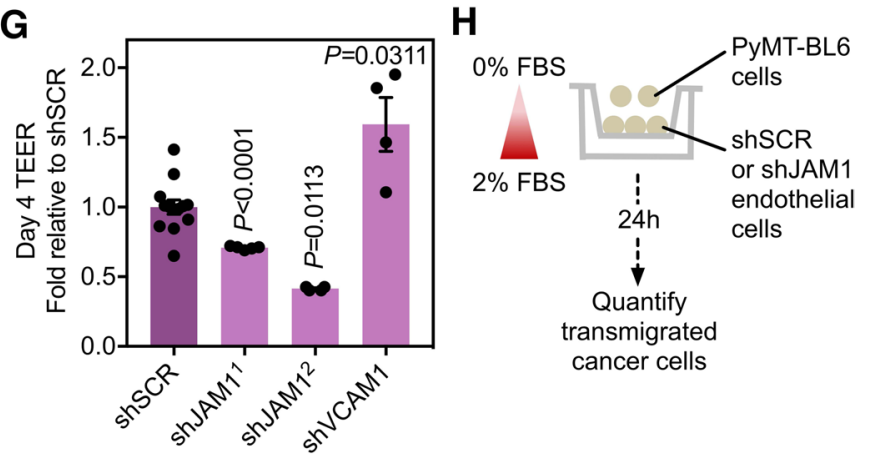

Extended Data Fig. 2 | JAM1 is sufficient to regulate vascular permeability. a, Schematic illustration of the in vitro TransEndothelial Electrical Resistance (TEER) assay. b. Schematic illustration of the in vitro dextran-FITC permeability assay. c, Representative immunofluorescence image for Fig. 2l, showing JAM1+CD31+ cells in $\mathrm{LF}(n=n=4$ mice) and HF ( $n=7$ mice) lung tissue. $\mathbf{d}$, Representative immunofluorescence images for Fig. 21 showing adhesion protein staining in CD31+ cells in LF ( $n=4$ mice) and HF lung tissue ( $N$-cadherin, $n=4$ mice; all others, $n=5$ mice). e, Top: Western blot analysis confirming knockdown of VCAM1 protein in HMEC endothelial cells via infection with shRNA targeting VCAM1. Knockdown was confirmed in 2 independent blots. Bottom: TEM quantification in which monolayers were formed with either shSCR or shVCAM1 HMEC endothelial cells prior to assessing Py 230 breast cancer transmigration. $n=4$ Transwells per group representing individual experimental replicates; mean \pm SEM; two-tailed Student's t-test. f, Representative western blot confirming knockdown of JAM1 protein in HMEC endothelial cells after infection with 2 shRNA constructs. Knockdown was confirmed in 2 independent blots. g, Quantification of TEER across HMEC monolayers genetically modified to express either a JAM1 shRNA (shJAM11, $\mathrm{n}=5$ Transwells; shJAM12 $\mathrm{n}=4$ Transwells), a VCAM1 shRNA (shVCAM1; $\mathrm{n}=4$ Transwells) or a scramble control shRNA (shSCR). Mean \pm SEM; two-tailed Student's t-test. Transwells represent individual experimental replicates with similar results. $\mathbf{h}$, Schematic illustration of the in vitro TEM assay corresponding to Fig. 20, in which HMEC monolayers genetically modified to express a JAM1 shRNA (shJAM1') or a scramble control shRNA (shSCR) were formed prior to assessing Py230 breast cancer transmigration. 
A

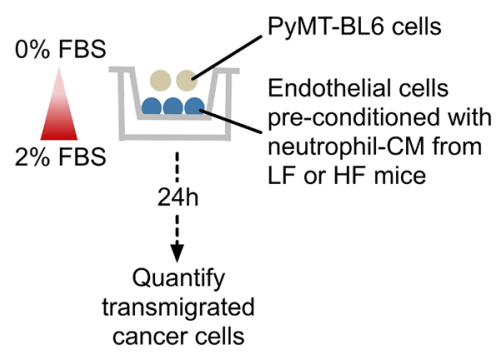

B

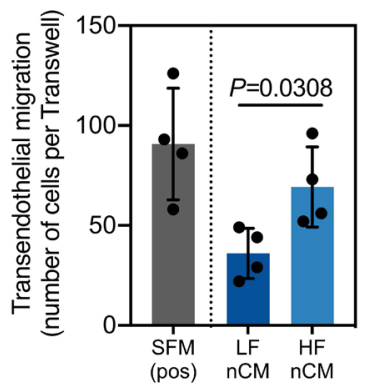

C

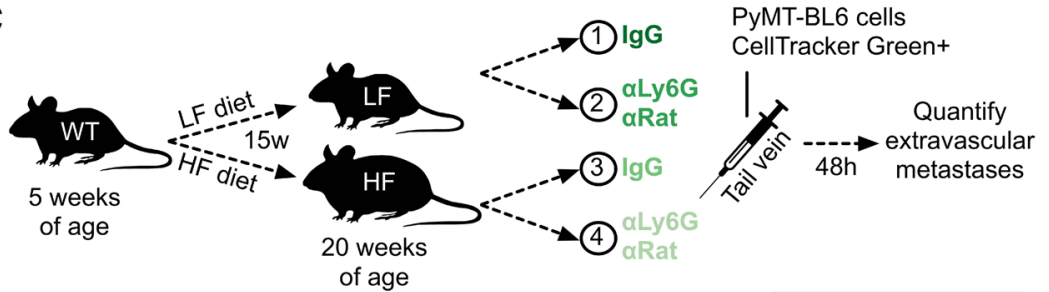

D
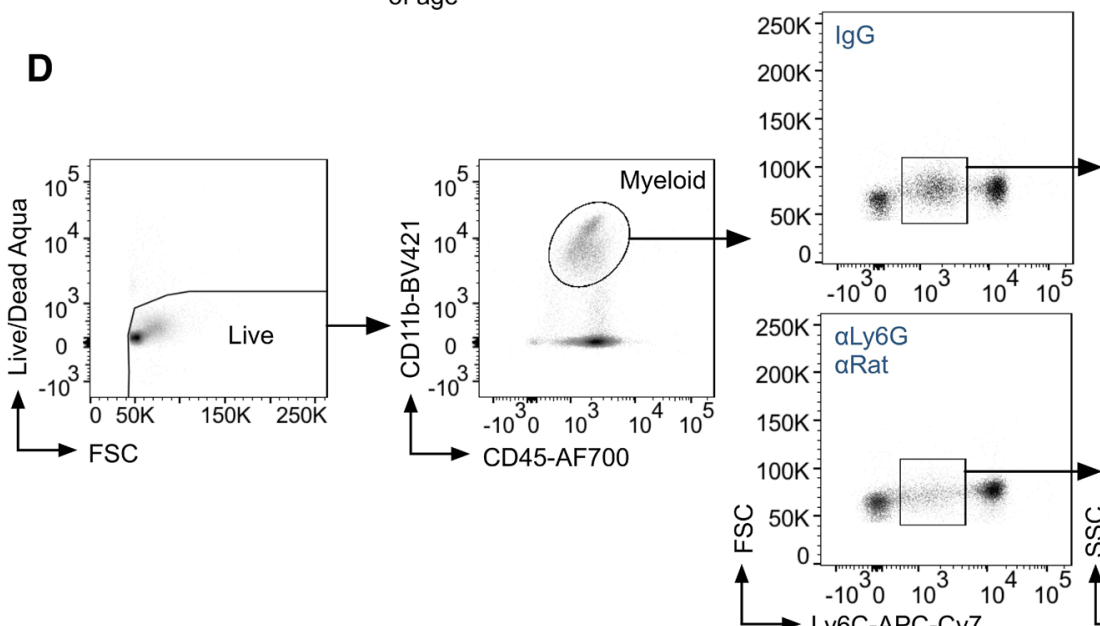

Ly6C-APC-Cy7
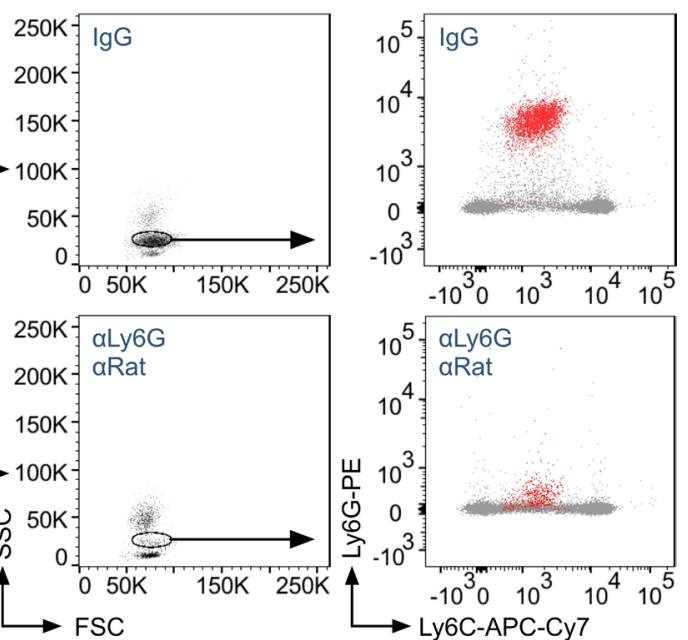

Extended Data Fig. 3 | Neutrophil depletion in vivo. a, Schematic illustration of a modified in vitro TEM assay, in which endothelial cells are pre-treated with neutrophil conditioned media ( $\mathrm{nCM}$ ) generated from bone marrow-derived neutrophils from LF or HF mice. b, Quantification of control TEM assay where HMEC endothelial cells are pre-treated for 24 hours with serum-free media (SFM), or nCM from either LF or HF mice. PyMT cells are then seeded in the upper chamber, and a 0-2\% FBS gradient is used to stimulate TEM. $n=4$ Transwells per condition representing experimental replicates using $\mathrm{nCM}$ from individual mice; mean \pm SEM; two-tailed Student's t-test. c, Schematic of trial design for in vivo 48h extravasation assay with antibody-based neutrophil depletion using ?Ly6G/?Rat or an IgG control. d, Flow cytometry confirmation that neutrophils are effectively depleted in response to ?Ly6G/?Rat treatment, corresponding to experiments in Fig. 3h,i. 
A

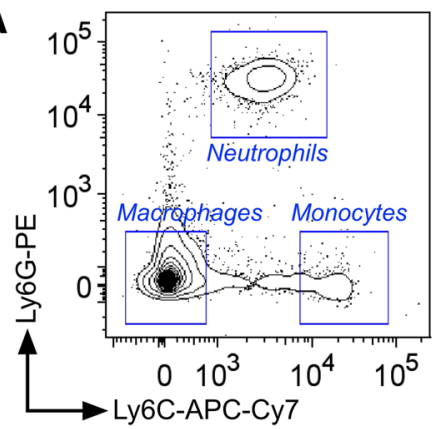

B

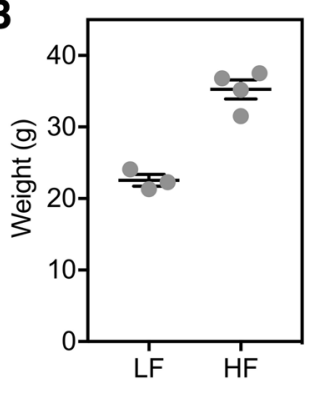

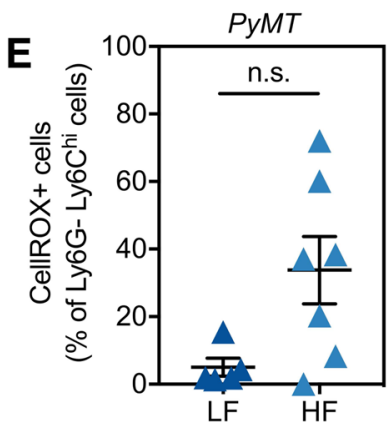
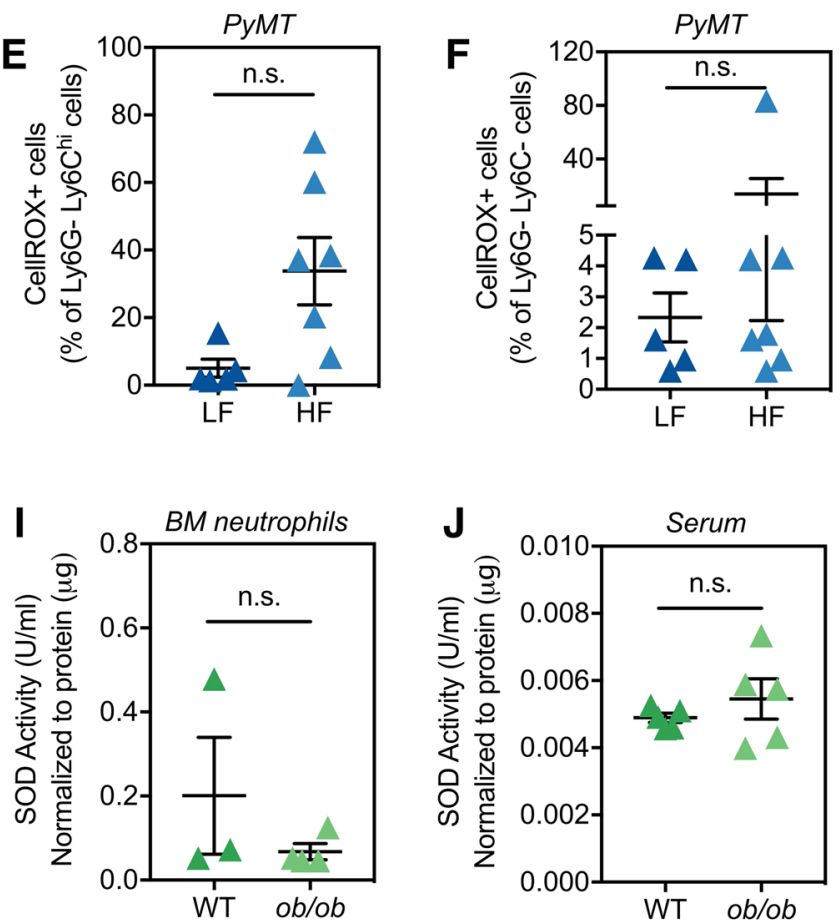

C

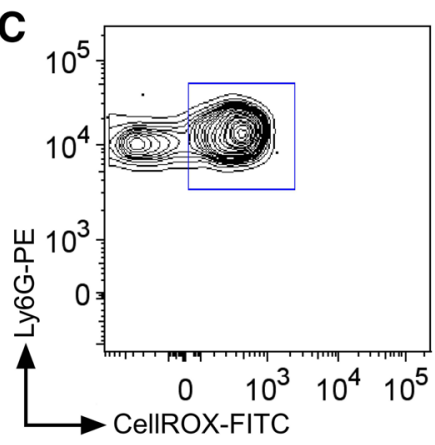

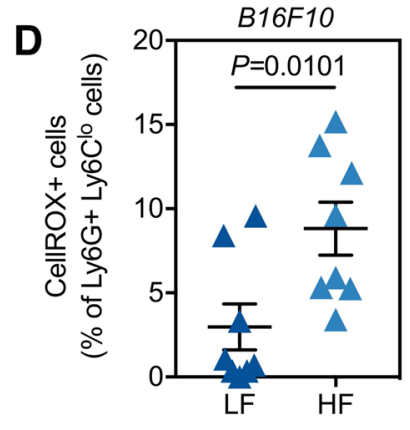
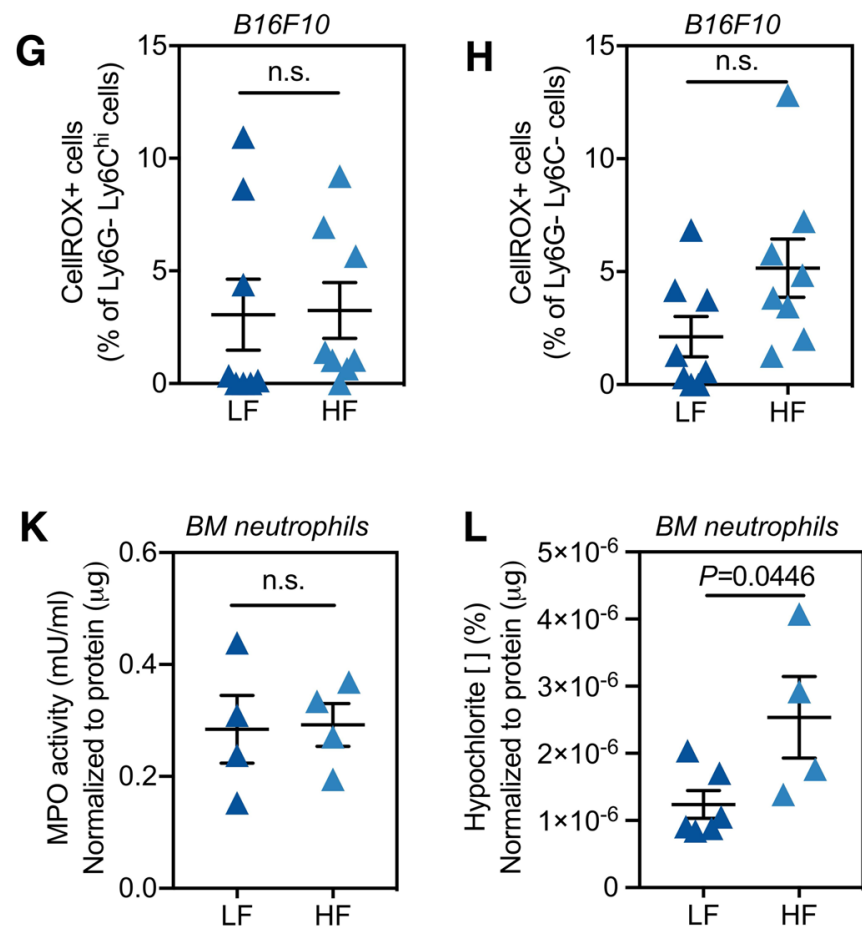

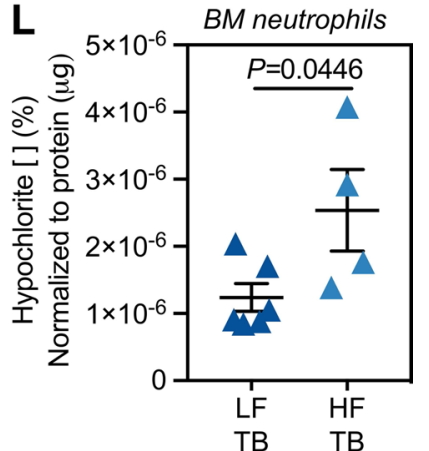

Extended Data Fig. 4 | Obesity is associated with enhanced ROS in neutrophils. a, Flow cytometry gating strategy for neutrophils, monocytes, and macrophages in lung. Gates were first drawn around CD45+ CD11b+ cells (not shown), followed by gating according to Ly6G and Ly6C as shown. CD45+ $\mathrm{CD}_{11} \mathrm{~b}^{+}$Ly6G ${ }^{+}$Ly6C $\mathrm{C}^{10}$ neutrophils were used for RNA-seq, corresponding to Fig. $4 a-d$. b. Weights of LF $(n=3$ mice) and HF ( $n=4$ mice) mice used for lung neutrophil RNA-sequencing. Mean \pm SEM. c, Neutrophil CellROX flow cytometry plot, corresponding to Fig. 4e. d, Quantification of CellROX+ neutrophils $\left(\mathrm{CD} 45^{+} \mathrm{CD}_{\left.11 b^{+} \mathrm{Ly} 6 \mathrm{G}^{+} \mathrm{Ly} 6 \mathrm{C}^{\circ}\right)}\right)$ in lungs from LF or HF B16F10 primary tumor-bearing mice. $\mathrm{n}=8$ mice per condition; mean \pm SEM; two-tailed Mann-Whitney test. e, Quantification of CellROX+ monocytes (CD45+ CD11b+ Ly6G- Ly6C hi) in lungs from LF or HF Py230 primary tumor-bearing mice. $\mathrm{LF}, \mathrm{n}=5$ mice; HF, $\mathrm{n}=7$ mice; mean \pm SEM; two-tailed Mann-Whitney test. f, Quantification of CellROX+ macrophages (CD45+ CD11b+ Ly6G- Ly6C-) in lungs from LF or HF Py230 primary tumor-bearing mice. LF, $n=5$ mice; $H F, n=7$ mice; mean \pm SEM; two-tailed Mann-Whitney test. $\mathbf{g}$, Quantification of CellROX ${ }^{+}$monocytes (CD45 ${ }^{+}$CD11 b $^{+}$Ly6G $^{-}$Ly6 $^{\text {hi }}$ ) in lungs from LF or HF B16F10 primary tumor-bearing mice. $n=8$ mice per condition; mean \pm SEM; two-tailed Mann-Whitney test. h, Quantification of CellROX+ macrophages (CD45+ CD11b+ Ly6G- Ly6C) in lungs from LF or HF B16F10 primary tumor-bearing mice. $\mathrm{n}=8$ mice per condition; mean $\pm \mathrm{SEM}$; two-tailed Student's t-test. $\mathbf{i}$, Quantification of SOD activity (U/ml) using bone marrow neutrophils isolated from WT or ob/ob mice. LF, $\mathrm{n}=3$ mice; HF, $\mathrm{n}=4$ mice; mean $\pm \mathrm{SEM}$; two-tailed Mann-Whitney test. $\mathbf{j}$, Quantification of SOD activity $(\mathrm{U} / \mathrm{ml})$ using serum isolated from WT or ob/ob mice. $\mathrm{n}=5$ mice per condition; mean $\pm \mathrm{SEM}$; two-tailed Student's t-test. k, Quantification of MPO activity $(\mathrm{mU} / \mathrm{ml})$ using bone-marrow neutrophils isolated from LF or HF mice. $\mathrm{n}=4$ mice per condition; mean $\pm \mathrm{SEM}$; two-tailed Student's t-test. I, Quantification of hypochlorite concentration in bone marrow neutrophils isolated from LF or HF mice harbouring E0771 mammary tumors (tumor-bearing; TB). LF, $\mathrm{n}=6$ mice; $\mathrm{HF}, \mathrm{n}=4$ mice; mean $\pm \mathrm{SEM}$; two-tailed Student's t-test. 
A

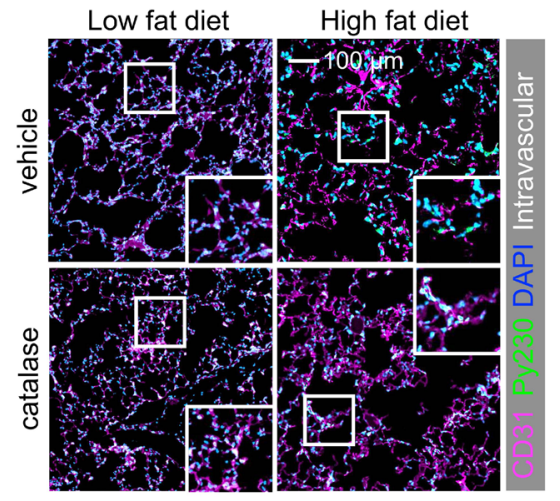

C

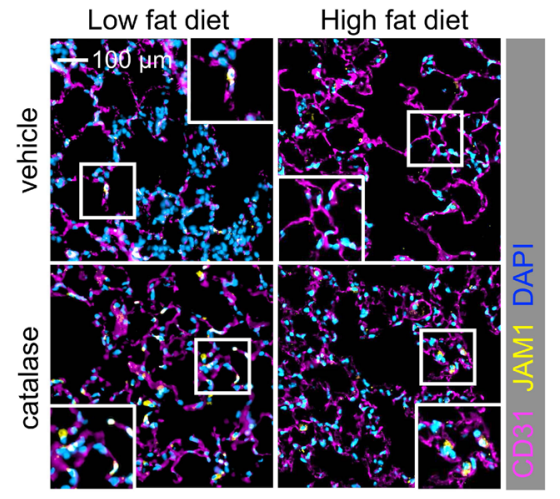

$\mathbf{E}$

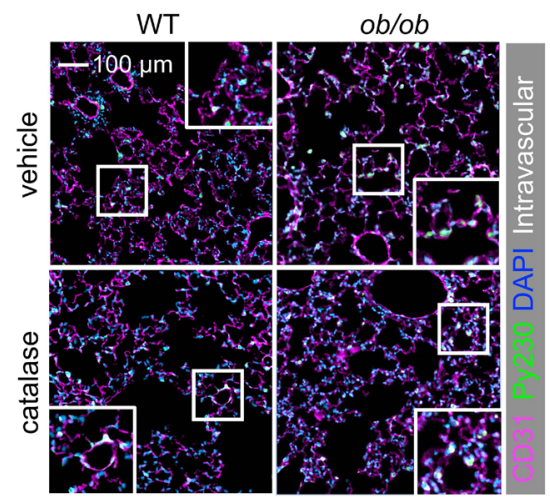

B

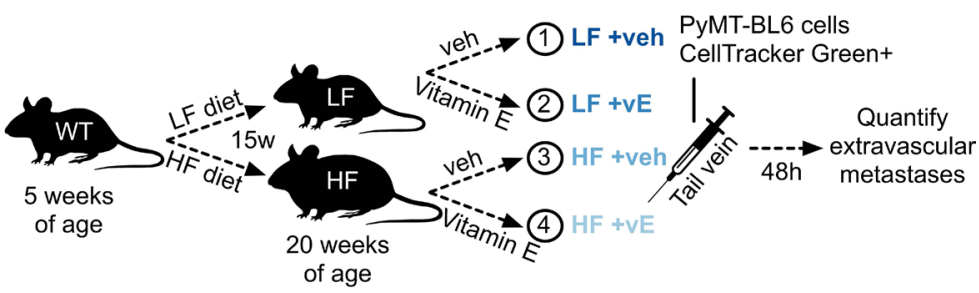

D

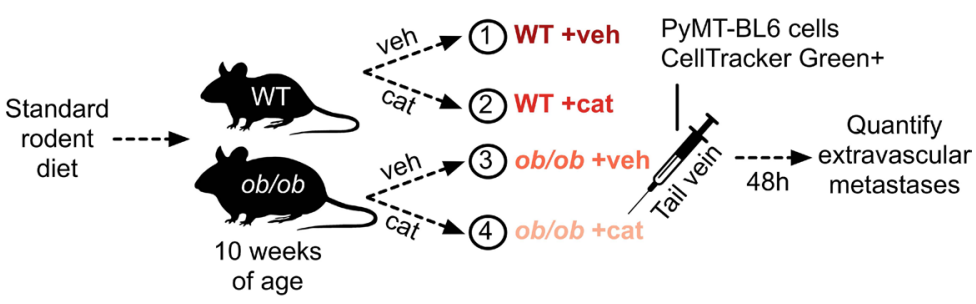

Extended Data Fig. $\mathbf{5}$ | Catalase reduces breast cancer extravasation to lung in obese hosts. a, Representative immunofluorescence image for data shown in Fig. $5 c$. LF + veh, $n=5$ mice; LF + cat, $n=4$ mice; HF + veh, $n=5$ mice; HF + cat, $n=4$ mice. $\mathbf{b}$, Schematic illustration of the in vivo extravasation assay corresponding to Fig. $5 \mathrm{~d}$. LF or HF mice were injected via tail vein with syngeneic breast cancer cells labelled with a green fluorescent CellTracker, treated with either vitamin $\mathrm{E}$ or vehicle and extravasation was quantified in lung after $48 \mathrm{~h}$ via fluorescence microscopy. $\mathbf{c}$, Representative immunofluorescence image for data shown in Fig. 5f. LF + veh, $n=6$ mice; $L F+$ cat, $n=4$ mice; $H F+$ veh, $n=8$ mice; HF + cat, $n=8$ mice. $\mathbf{d}$, Schematic illustration of the in vivo extravasation assay corresponding to Fig. $5 \mathrm{~g}, \mathrm{~h}$. WT or ob/ob mice were injected via tail vein with syngeneic breast cancer cells labelled with a green fluorescent CellTracker, treated with either catalase or vehicle and extravasation was quantified in lung after $48 \mathrm{~h}$ via fluorescence microscopy. $\mathbf{e}$, Representative immunofluorescence image for data shown in Fig. 5g. WT + veh, $\mathrm{n}=9$ mice; WT + cat, $\mathrm{n}=7$ mice; $o b / o b+$ veh, $\mathrm{n}=7$ mice; $o b / o b+c a t, \mathrm{n}=4$ mice. $\mathbf{f}$, Quantification of TEER across an HMEC monolayer genetically modified to express an shRNA against catalase (shCAT), versus a scramble control (shSCR). $n=4$ Transwells per condition representing individual experimental replicates with similar results; mean \pm SEM; two-tailed Student's t-test. 


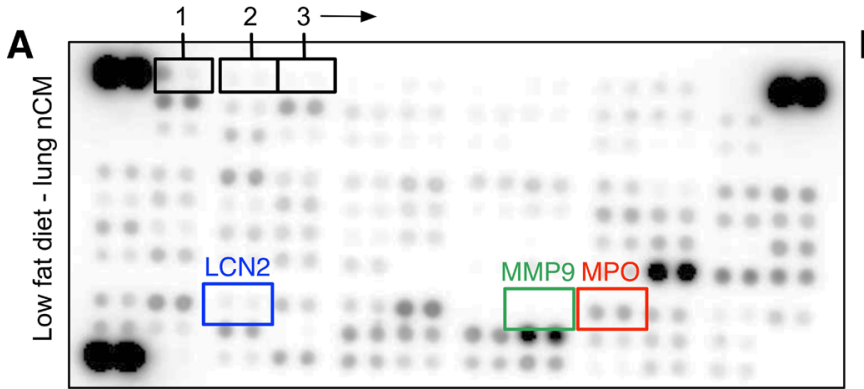

\begin{tabular}{|c|c|c|c|c|c|c|c|}
\hline 1 & AdipoQ & 29 & $\overline{C X C L 1}$ & 57 & IGFBP-6 & 85 & MMP-3 \\
\hline 2 & $A R$ & 30 & $\overline{C X C L 2}$ & 58 & IL-1a & 86 & MMP-9 \\
\hline 3 & Ang-1 & 31 & CXCL9 & 59 & IL-1B & 87 & MPO \\
\hline 4 & Ang-2 & 32 & CXCL10 & 60 & IL-1ra & 88 & OPN \\
\hline 5 & ANGPT-L3 & 33 & CXCL11 & 61 & IL-2 & 89 & OPG \\
\hline 6 & CD257 & 34 & CXCL13 & 62 & IL-3 & 90 & PD-ECGF \\
\hline 7 & CD93 & 35 & CXCL16 & 63 & IL-4 & 91 & PDGF-BB \\
\hline 8 & CCL2 & 36 & Cystatin C & 64 & IL-5 & 92 & SAP \\
\hline 9 & CCL3 & 37 & DKK-1 & 65 & IL-6 & 93 & TSG-14 \\
\hline 10 & CCL5 & 38 & DPPIV & 66 & IL-7 & 94 & OSF-2 \\
\hline 11 & CCL6 & 39 & EGF & 67 & IL-10 & 95 & DLK-1 \\
\hline 12 & CCL11 & 40 & CD105 & 68 & IL-11 & 96 & MRP \\
\hline 13 & CCL12 & 41 & \begin{tabular}{|l|} 
Endostatin \\
\end{tabular} & 69 & $11-12$ p40 & 97 & NARC-1 \\
\hline 14 & CCL17 & 42 & AHSG & 70 & IL-13 & 98 & RAGE \\
\hline 15 & CCL19 & 43 & FGF-1 & 71 & IL-15 & 99 & RBP4 \\
\hline 16 & $\mathrm{CCL} 20$ & 44 & FDF-21 & 72 & IL-17A & 100 & Reg3G \\
\hline 17 & CCL21 & 45 & Flt3lg & 73 & IL-22 & 101 & ADSF \\
\hline 18 & CCL22 & 46 & Gas 6 & 74 & IL-23 & 102 & CD62E \\
\hline 19 & CD14 & 47 & G-CSF & 75 & \begin{tabular}{|l|} 
IL-27 p28 \\
\end{tabular} & 103 & CD62P \\
\hline 20 & CD40 & 48 & GDF-15 & 76 & IL-28A/B & 104 & PAl-1 \\
\hline 21 & CD160 & 49 & GM-CSF & 77 & IL-33 & 105 & PEDF \\
\hline 22 & TIG-2 & 50 & HGF & 78 & LDLR & 106 & Tpo \\
\hline 23 & CHI3L1 & 51 & ICAM-1 & 79 & Leptin & 107 & TIM-1 \\
\hline 24 & TF & 52 & \begin{tabular}{|l|} 
INF-gamma \\
\end{tabular} & 80 & LIF & 108 & TNF-a \\
\hline 25 & C5/C5a & 53 & IGFBP-1 & 81 & Lipocalin-2 & 109 & CD106 \\
\hline 26 & Adipsin & 54 & IGFBP-2 & 82 & LIX & 110 & VEGF \\
\hline 27 & CRP & 55 & IGFBP-3 & 83 & M-CSF & 111 & CCN4 \\
\hline 28 & CX3CL1 & 56 & IGFBP-5 & 84 & MMP-2 & \multicolumn{2}{|c|}{ norm to neg control } \\
\hline
\end{tabular}
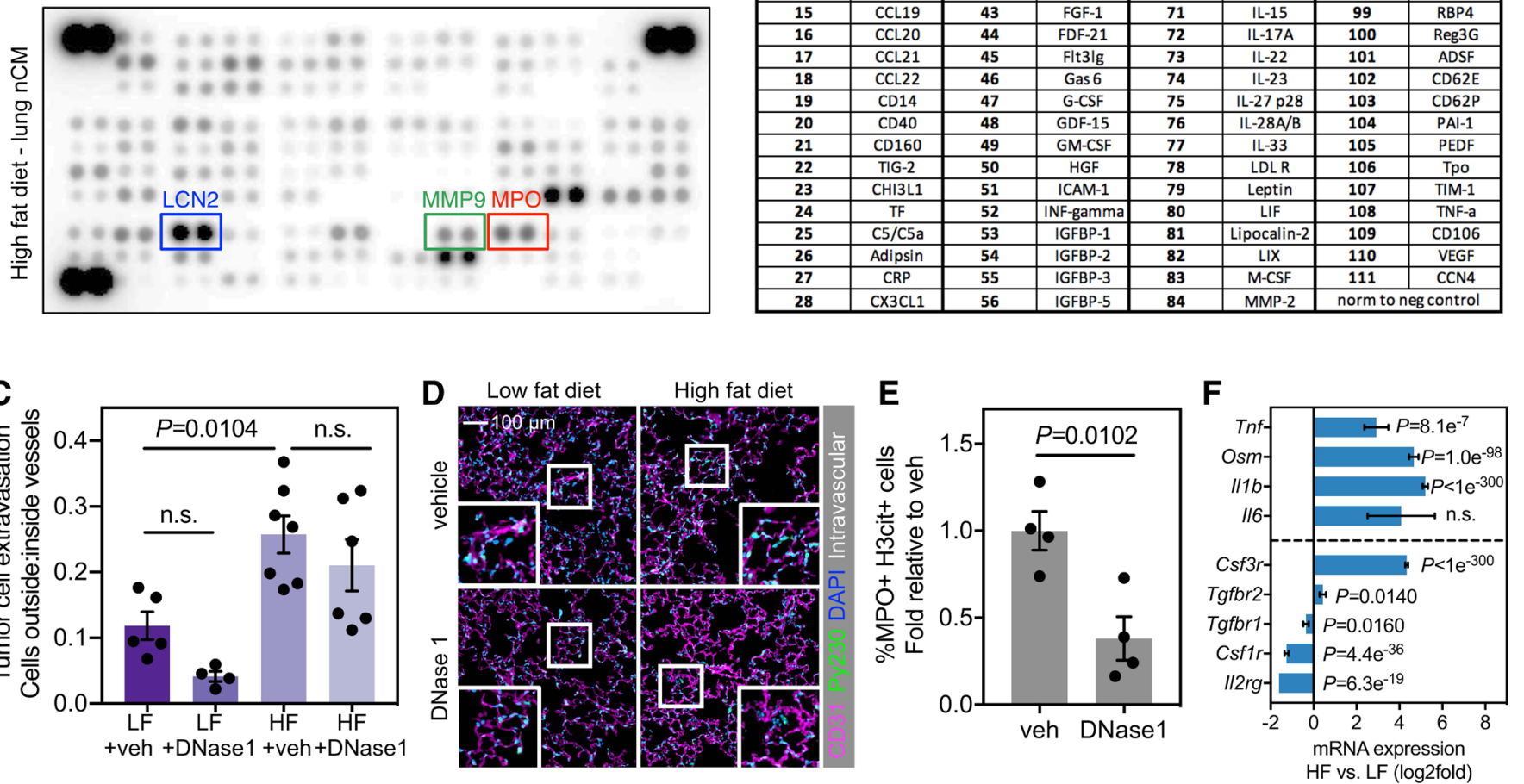

Extended Data Fig. 6 | Obesity increases MMP9 from neutrophils. a, Cytokine array from lung neutrophil conditioned media ( $\mathrm{nCM}$ ) obtained from LF or HF mice. MMP9 and LCN2 were the top two differences observed. b, Outline of 111 cytokine array membrane targets, corresponding to a. c, Immunofluorescence quantification of Py 230 cancer cell extravasation in lung tissue from LF or HF mice treated with DNase1 or vehicle. LF + veh, $n=5$ mice; $L F+D N a s e 1, n=n=4$ mice; HF + veh, $n=7$ mice; HF + DNase1, $n=6$ mice; mean \pm SEM; One-way ANOVA with Bonferroni multiple comparisons test. d, Representative immunofluorescence image for data shown in c. e, Immunofluorescence quantification of MPO and $\mathrm{H} 3 c$ it in lung tissue from obese (HF) mice treated with DNase1 or vehicle. $n=4$ mice per group; mean \pm SEM; two-tailed Student's t-test. $\mathbf{f}$, RNA-seq differentially expressed genes in $\mathrm{HF}(\mathrm{n}=4$ mice) relative to $\mathrm{LF}$ ( $n=3$ mice) lung neutrophils, showing representative genes relevant to cell cycle progression during metastasis. Data are displayed as log2fold HF vs LF with standard error. 
A

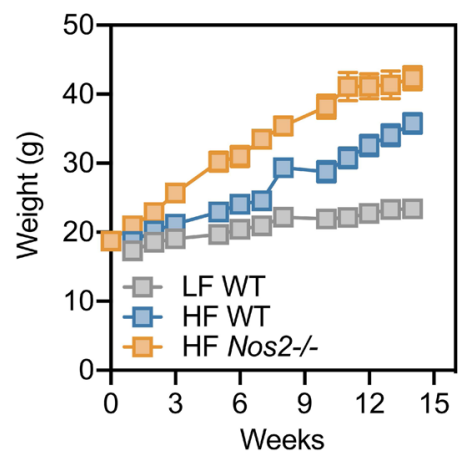

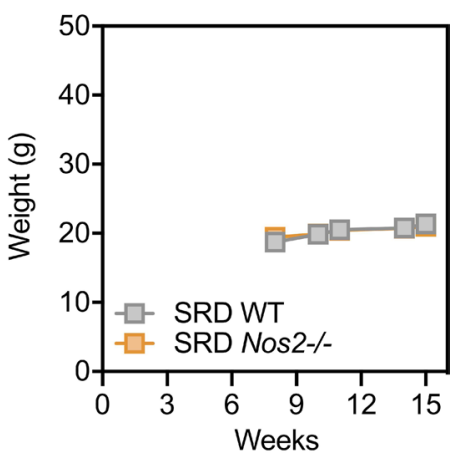

Extended Data Fig. 7 | Diet-induced weight changes between WT and Nos2-/- mice. a, Weight curves comparing wild type (WT) and Nos2-/- mice enrolled on LF, HF or SRD diet from 5-20 weeks of age (15 weeks total). LF WT, $n=10$ mice; HF WT, $n=10$ mice; HF Nos2 $-/-n=5$ mice; SRD WT, $n=10$ mice; SRD Nos2-/-, $\mathrm{n}=10$ mice; mean \pm SEM. 
A

\begin{tabular}{|c|c|c|c|c|}
\hline & BMI & Primary cancer & Sex & Age \\
\hline 1 & $19.9^{*}$ & Prostate & $M$ & 75 \\
\hline 2 & $21.3^{*}$ & CRC & $M$ & 77 \\
\hline 3 & $22.1^{*}$ & Colon & $M$ & 86 \\
\hline & $24.1^{*}$ & Bladder & $M$ & 78 \\
\hline 5 & $25.4^{*}$ & Brain & $\mathrm{F}$ & 73 \\
\hline 6 & $25.4^{*}$ & Prostate & $M$ & 81 \\
\hline 7 & $25.5^{*}$ & CRC & $M$ & 80 \\
\hline 8 & $25.6^{\star}$ & Colon & M & 58 \\
\hline 9 & $26.3^{*}$ & Breast & $\mathrm{F}$ & 63 \\
\hline & $26.5^{*}$ & CRC & M & 76 \\
\hline & $26.7^{*}$ & Colon & $\mathrm{F}$ & 46 \\
\hline & 27.7 & CRC & $M$ & 79 \\
\hline & 27.7 & CRC & $M$ & 79 \\
\hline & $28.2^{*}$ & Colon & $M$ & 72 \\
\hline & $28.4^{*}$ & Esophageal & $M$ & 66 \\
\hline & 29.4 & CRC & $M$ & 79 \\
\hline & $29.6^{*}$ & Colon & $M$ & 59 \\
\hline & $29.8^{*}$ & CRC & $\mathrm{F}$ & 48 \\
\hline & 29.9 & CRC & $M$ & 79 \\
\hline & $33.2^{*}$ & CRC & $M$ & 79 \\
\hline & 33.4 & CRC & $M$ & 79 \\
\hline & $36.3^{*}$ & Prostate & $M$ & 78 \\
\hline
\end{tabular}

B

\begin{tabular}{|c|c|c|c|}
\hline & Metal & Target & Clone \\
\hline 1 & $141 \mathrm{Pr}$ & aSMA & $1 \mathrm{~A} 4$ \\
\hline 2 & $142 \mathrm{Nd}$ & CD56 & EP2567Y \\
\hline 3 & $143 \mathrm{Nd}$ & Vimentin & RV202 \\
\hline 4 & $144 \mathrm{Nd}$ & CD14 & EPR3652 \\
\hline$v_{1}$ & $145 \mathrm{Nd}$ & MPO & EPR20257 \\
\hline 6 & $146 \mathrm{Nd}$ & MMP9 & EP1254 \\
\hline 7 & $147 \mathrm{Sm}$ & CD31 & EP3095 \\
\hline 8 & $148 \mathrm{Nd}$ & H3cit & EPR20358-120 \\
\hline 9 & $149 \mathrm{Sm}$ & Hif1a & EP1215Y \\
\hline 0 & $150 \mathrm{Nd}$ & PD-L1 & SP142 \\
\hline 1 & $151 \mathrm{Eu}$ & LAMP1 & H4A3 \\
\hline 2 & $152 \mathrm{Sm}$ & CD45 & CD45-2B11 \\
\hline 3 & 153Eu & JAM1 & EP1042Y \\
\hline 4 & $154 \mathrm{Sm}$ & CD11c & EP1347Y \\
\hline 5 & $155 \mathrm{Gd}$ & FOXP3 & 236A/E7 \\
\hline 6 & $156 \mathrm{Gd}$ & $\mathrm{CD} 4$ & EPR6855 \\
\hline 7 & $158 \mathrm{Gd}$ & CCR7 & Y59 \\
\hline 8 & $159 \mathrm{~Tb}$ & CD68 & KP1 \\
\hline 19 & $160 \mathrm{Gd}$ & Catalase & EP1929Y \\
\hline
\end{tabular}

\begin{tabular}{|c|c|c|c|}
\hline & Metal & Target & Clone \\
\hline 20 & 161Dy & CD20 & $\mathrm{H} 1$ \\
\hline 21 & 162Dy & CD8a & C8/144 \\
\hline 2 & 163Dy & iNOS & EPR16635 \\
\hline & 164Dy & CD163 & EPR19518 \\
\hline & $165 \mathrm{Ho}$ & PD1 & EPR4877(2) \\
\hline & $166 \mathrm{Er}$ & CD45RA & HI100 \\
\hline & $167 \mathrm{Er}$ & CD39 & EPR20627 \\
\hline & $168 \mathrm{Er}$ & Ki67 & B56 \\
\hline & $169 \mathrm{Tm}$ & Collagen I & polyclonal \\
\hline & $170 \mathrm{Er}$ & CD3 & polyclonal \\
\hline & $171 \mathrm{Yb}$ & CD27 & EPR8569 \\
\hline & $172 \mathrm{Yb}$ & $\mathrm{CC} 3$ & 5A1E \\
\hline & $173 \mathrm{Yb}$ & CD45RO & UCHL1 \\
\hline & $174 Y \mathrm{Yb}$ & HLA-DR & EPR3692 \\
\hline & 175Lu & Pan-CK & AE1/AE3 \\
\hline & $176 \mathrm{Yb}$ & Histone H3 & $\mathrm{D} 1 \mathrm{H} 2$ \\
\hline & $191 / r$ & DNA1 & \\
\hline & $193 / r$ & DNA2 & \\
\hline
\end{tabular}

C Staining workflow

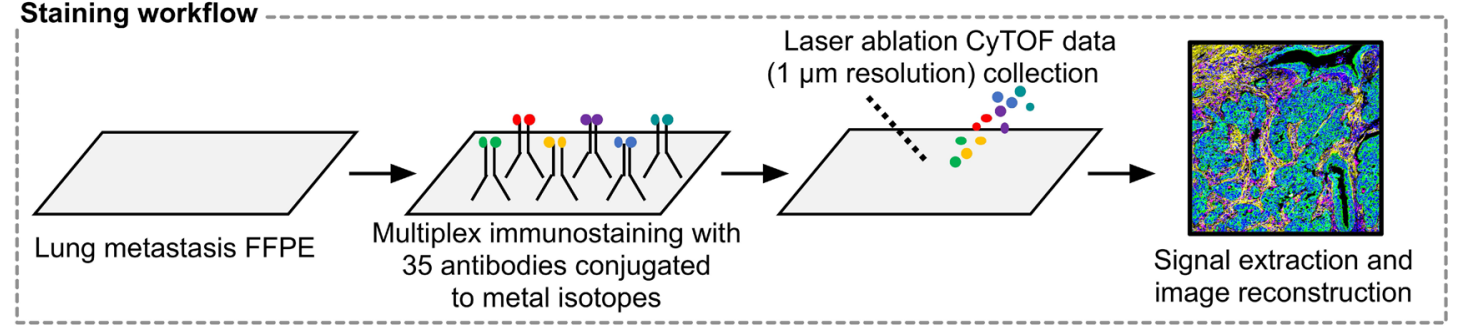

D

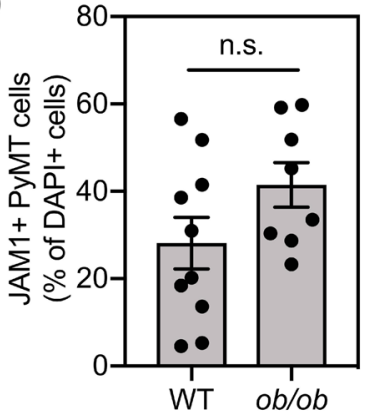

E

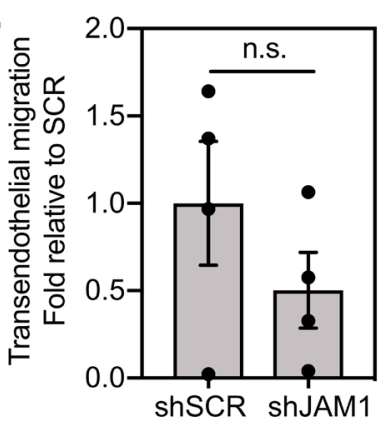

$\mathbf{F}$

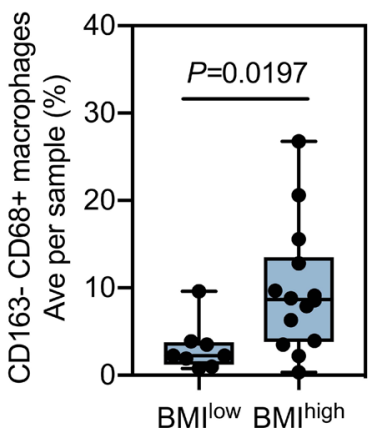

Extended Data Fig. 8 | Imaging mass cytometry (IMC) allows for single cell and spatial analysis. a, Patient information (BMI and primary cancer type) for samples analyzed by IMC. Patients with an asterisk indicate those used for immunofluorescence validation. Patients with $\mathrm{BMI}<25.9$ were assigned to the $\mathrm{BMI}^{\text {low }}$ group (ave $\mathrm{BMI}=24$ ), and patients with a $\mathrm{BMI}>26$ were assigned to the $\mathrm{BM} \mathrm{l}^{\text {high }}$ group (ave $\mathrm{BMI}=30$; black line). $\mathbf{b}$, IMC antibody panel information. c, Schematic of IMC staining workflow. Tissue sections were stained with antibody panel (as in b), and subject to CyTOF acquisition using a Hyperion imaging system. d, Immunofluorescence quantification of JAM1+ PyMT tumor cells in lung tissue from WT or ob/ob mice. WT, $\mathrm{n}=10$ mice; $o b / o b$, $\mathrm{n}=8$ mice; mean $\pm \mathrm{SEM}$; two-tailed Student's t-test. e, Quantification of TEM of PyMT breast cancer cells infected with an shRNA against Jam 1 (shJAM1) or scramble control (shSCR). $n=4$ Transwells per group representing individual experimental replicates; mean \pm SEM; two-tailed Student's T test. $\mathbf{f}$, Quantification of the average number of total CD163- CD68 ${ }^{+}$M1-like macrophages in BMlow ( $n=8$ patients) and BMl high ( $n=14$ patients) lung metastasis samples. Box $=$ median \pm interquartile range, whiskers $=$ min-max, all datapoints shown; two-tailed Mann-Whitney test. 


\section{Reporting Summary}

Nature Research wishes to improve the reproducibility of the work that we publish. This form provides structure for consistency and transparency in reporting. For further information on Nature Research policies, see Authors \& Referees and the Editorial Policy Checklist.

\section{Statistics}

For all statistical analyses, confirm that the following items are present in the figure legend, table legend, main text, or Methods section.

n/a Confirmed

$\bigotimes$ The exact sample size $(n)$ for each experimental group/condition, given as a discrete number and unit of measurement

$\square$ A statement on whether measurements were taken from distinct samples or whether the same sample was measured repeatedly

The statistical test(s) used AND whether they are one- or two-sided

Only common tests should be described solely by name; describe more complex techniques in the Methods section.

$\square$ A description of all covariates tested

$\square \bigotimes$ A description of any assumptions or corrections, such as tests of normality and adjustment for multiple comparisons

$\square$ A

A full description of the statistical parameters including central tendency (e.g. means) or other basic estimates (e.g. regression coefficient) AND variation (e.g. standard deviation) or associated estimates of uncertainty (e.g. confidence intervals)

For null hypothesis testing, the test statistic (e.g. $F, t, r$ ) with confidence intervals, effect sizes, degrees of freedom and $P$ value noted Give $P$ values as exact values whenever suitable.

Х $\square$ For Bayesian analysis, information on the choice of priors and Markov chain Monte Carlo settings

Х $\square$ For hierarchical and complex designs, identification of the appropriate level for tests and full reporting of outcomes

$\square \bigotimes$ Estimates of effect sizes (e.g. Cohen's $d$, Pearson's $r$ ), indicating how they were calculated

\section{Our web collection on statistics for biologists contains articles on many of the points above.}

\section{Software and code}

Policy information about availability of computer code

Data collection

Information for data collection can be found in the Methods section. Data collection for imaging mass cytometry was performed using the Hyperion Imaging System CyTOF Software version 6.7.1014. RNA-sequencing was collected with an Illumina HISeq 2000 and reads were mapped to the mouse genome using STAR 2.6.1b.

Data analysis

The following was used for data analysis in this study: RStudio (version 3.6.1), Fastp (version 0.20.0), STAR aligner (version 2.6.1b), Sambamba (version 0.7.0), featureCounts (version 2.0.0), DESeq2 (version 1.24.0), biomaRt (version 2.40.4), Ingenuity Pathway Analysis (version 01-13), Gene Set Enrichment Analysis (version 4.1.0), MSigDB (version 7.2), DoRothEA (version 1.3.0), GraphPad Prism Pro (versions 7 and 8) , HALO (version 3.0.311.195). All experimental data statistics calculations can be found in Supplementary Table 2, along with a statistics 'decision tree'. Code for IMC cell segmentation was written in Matlab (version 7.10) and is available upon request.

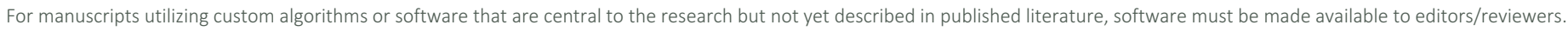
We strongly encourage code deposition in a community repository (e.g. GitHub). See the Nature Research guidelines for submitting code \& software for further information.

Data

Policy information about availability of data

All manuscripts must include a data availability statement. This statement should provide the following information, where applicable:

- Accession codes, unique identifiers, or web links for publicly available datasets

- A list of figures that have associated raw data

- A description of any restrictions on data availability

All experimental data used for graphs and statistics are included in Supplementary Table 2. Any additional data that support the findings of this study are available from the corresponding author upon reasonable request. RNA-seq data that support the findings of this study have been deposited in the Gene Expression Omnibus under the accession codes GSE165442 (lung neutrophils, Figs. 4a-d and 6c and Extended Data Fig. 6f) and GSE165441 (lung endothelial cells, Fig. 2a-c). Databases used for RNA-seq analysis included MSigDB v7.2 http://www.gsea-msigdb.org/gsea/msigdb/index.jsp, DoRothEA v1.3.0 https://saezlab.github.io/dorothea/, and IPA 


\section{Field-specific reporting}

Please select the one below that is the best fit for your research. If you are not sure, read the appropriate sections before making your selection.

\section{$\bigotimes$ Life sciences $\quad \square$ Behavioural \& social sciences $\square$ Ecological, evolutionary \& environmental sciences}

For a reference copy of the document with all sections, see nature.com/documents/nr-reporting-summary-flat.pdf

\section{Life sciences study design}

All studies must disclose on these points even when the disclosure is negative.

Sample size Sample sizes were chosen based on power of 0.9 to detect a difference of $>1.5$ standard deviations between LF vs. HF means with $95 \%$ confidence. However, in most cases, previous data was sufficient to inform sample size for subsequent experiments.

Data exclusions All exclusion criteria were pre-established: Mice on a low fat diet were excluded if they were heavier than $28 \mathrm{~g}$, and mice on a high fat diet were excluded if they were less than $28 \mathrm{~g}$.

Replication

All attempts at replication were successful: All western blots were performed 3 or more times with similar results; all flow cytometry experiments were repeated 3 or more times with similar results; all animal trials were repeated with at least $n=4$ in at least 2 independent cohorts with similar results; all in vitro assays were repeated in at least 3 independent experiments with similar results.

Randomization When comparing treatment efficacy in lean/obese animals (Fig. 3a, 5a, 6l, 7b, Supplementary Fig. 3c, 5b, 5d) allocation of mice to specific treatment groups ensured similar distribution of body weight. For all other experiments, no specific randomization method was followed as this was irrelevant to in vitro assays and analyses performed on human samples (body mass index pre-determined patient allocation).

Blinding

No specific blinding method was followed in this study (not possible for mouse studies based on weight). For all experiments, automated quantitative methods were used to eliminate subjective interpretation of data.

\section{Reporting for specific materials, systems and methods}

We require information from authors about some types of materials, experimental systems and methods used in many studies. Here, indicate whether each material, system or method listed is relevant to your study. If you are not sure if a list item applies to your research, read the appropriate section before selecting a response.

\begin{tabular}{|c|c|c|c|}
\hline \multicolumn{2}{|r|}{ Materials \& experimental systems } & \multicolumn{2}{|c|}{ Methods } \\
\hline $\mathrm{n} / \mathrm{a}$ & Involved in the study & $\mathrm{n} / \mathrm{a}$ & Involved in the study \\
\hline L & $\bigotimes$ Antibodies & Х & $\square$ ChIP-seq \\
\hline ᄂ & Eukaryotic cell lines & $\square$ & \ Flow cytometry \\
\hline$\bigotimes$ & $\square$ Palaeontology & Х & $\square$ MRI-based neuroimaging \\
\hline 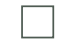 & Х Animals and other organisms & & \\
\hline \begin{tabular}{|c} 
\\
\end{tabular} & $\bigotimes$ Human research participants & & \\
\hline$\bigotimes$ & $\square$ Clinical data & & \\
\hline
\end{tabular}

\section{Antibodies}

Antibodies used

All antibodies used in this study were titrated for each lot. Information about all antibody dilutions, clones, etc can be found in Supplementary Table 1 and Supplementary Figure 8B, and in the Methods section. All antibodies are commercially available. A complete list is below:

Antibody / Clone / Species / Manufacturer / Catalogue No. (dilution)

IF/WB antibodies:

CD31 / Polyclonal / Mouse-Rat / R\&D / AF3628-SP (1:100 (IF); 1:500 (WB))

ZO-1 / Polyclonal / Human-Mouse / Abcam / ab96587 (1:100 (IF); 1:3000 (WB))

VE-Cadherin / BV13 / Mouse / Abcam / ab91064 (1:100 (IF); 1:500 (WB))

NG2 / 546930 / Mouse / R\&D / MAB6689-SP (1:100 (IF); 1:1000 (WB))

N-Cadherin / Polyclonal / Human-Mouse-Rat / Abcam / ab18203 (1:100 (IF); 1:500 (WB))

B-Catenin / Polyclonal / Human-Mouse-Rat / Abcam / ab16051 (1:100 (IF); 1:1000 (WB))

VCAM1 / EPR5038(2) / Human-Mouse-Rat / Abcam / ab174279 (1:100 (IF); 1:2000 (WB))

E-Selectin / Polyclonal / Human-Mouse / Abcam / ab18981 (1:100 (IF); 1:300 (WB))

Occludin / EPR20992 / Human-Mouse-Rat / Abcam / ab216327 (1:100 (IF); 1:1000 (WB))

ICAM1 / YN1/1.7.4 / Mouse / Abcam / ab119871 (1:100 (IF); 1:500 (WB))

JAM1 / Polyclonal / Human-Mouse-Rat / Abcam / ab180821 (1:400 (IF); 1:500 (WB)) 
p-VE-Cadherin / Polyclonal / Human-Mouse-Rat / Sigma / SAB4504676-100UG (1:500)

HSP90 / C45G5 / Human-Mouse-Rat / Cell Signaling / 4877 (1:1000)

$\beta$-actin / C4/actin / Human-Mouse-Rat / BD / 612656 (1:5000)

MPO / Polyclonal / Human-Mouse / R\&D / AF3367 (1:100 (IF); 1:500 (WB))

H3cit (R2+R8+R17) / Polyclonal / Human-Mouse-Rat / Abcam / ab5103 (1:100)

Chlorotyrosine / Polyclonal / Human / Hycult Biotech / HP5002 (1:50)

FACS/flow antibodies:

CD45 / 30-F11 / Mouse / BioLegend / 103127 (1:1000)

CD11b / M1/70 / Human-Mouse / BioLegend / 101235 (1:500)

Ly6C / HK1.4 / Mouse / BioLegend / 128025 (1:500)

Ly6G / 1A8 / Mouse / BD / 551461 (1:200)

CD31 / MEC 13.3 / Mouse / BioLegend / 102522 (1:200)

In vivo neutrophil depletion antibodies:

Gr1 / RB6-8C5 / Mouse / Tonbo Biosciences / 40-5931 (6 mg/kg)

IgG2b / MPC-11 / Mouse / Tonbo Biosciences / 40-4732 (6 mg/kg)

Ly6G / 1A8 / Mouse / BioXcell / BE0075-1 (4 mg/kg)

IgG2a / MAR 18.5 / Rat / BioXcell / BE0122 (4 mg/kg)

IgG2a / 2A3 / n/a (anti-trinitrophenol) / BioXcell / BE0089 (4 mg/kg)

IMC antibodies:

aSMA / 1A4 / Human / Fluidigm / 3141017D (1:100)

CD56 / EP2567Y / Human / Abcam / ab214435 (1:100)

Vimentin / RV202 / Human / Fluidigm / 3143029D (1:100)

CD14 / EPR3652 / Human / Abcam / ab209971 (1:50)

MPO / EPR20257 / Human / Abcam / ab221847 (1:50)

MMP9 / EP1254 / Human / Abcam / ab204850 (1:50)

CD31 / EP3095 / Human / Abcam / ab226157 (1:100)

H3cit / EPR20358-120 / Human / Abcam / ab232938 (1:50)

Hif1a / EP1215Y / Human / Abcam / ab210073 (1:100)

PD-L1 / SP142 / Human / Abcam / ab236238 (1:50)

LAMP1 / H4A3 / Human / Fluidigm / 3151021D (1:100)

CD45 / CD45-2B11 / Human / Fluidigm / 3152016D (1:100)

JAM1 / EP1042Y / Human / Abcam / ab232338 (1:50)

CD11c / EP1347Y / Human / Abcam / ab216655 (1:50)

FOXP3 / 236A/E7 / Human / Abcam / ab96048 (1:50)

CD4 / EPR6855 / Human / Fluidigm / 3156033D (1:100)

CCR7 / Y59 / Human / Abcam / ab271833 (1:800)

CD68 / KP1 / Human / Abcam / ab233172 (1:100)

Catalase / EP1929Y / Human / Abcam / ab227116 (1:50)

CD20 / H1 / Human / Fluidigm / 3161029D (1:100)

CD8a / C8/144 / Human / Abcam / ab213020 (1:100)

iNOS / EPR16635 / Human / Abcam / ab213987 (1:25)

CD163 / EPR19518 / Human / Abcam / ab213612 (1:100)

PD1 / EPR4877(2) / Human / Fluidigm / 3165039D (1:50)

CD45RA / HI100 / Human / Fluidigm / 3166028D (1:100)

CD39 / EPR20627 / Human / Abcam / ab236038 (1:100)

Ki67 / B56 / Human / Fluidigm / 3168007B (1:100)

Collagen 1 / Polyclonal / Human / Fluidigm / 3169023D (1:100)

CD3 / Polyclonal / Human / Fluidigm / $3170019 D$ (1:100)

CD27 / EPR8569 / Human / Fluidigm / 3171024D (1:100)

CC3 / 5A1E / Human / Fluidigm / $3172027 D$ (1:50)

CD45RO / UCHL1 / Human / Fluidigm / 3173016D (1:100)

HLA-DR / EPR3692 / Human / Abcam / ab209968 (1:100)

Pan-CK / AE1/AE3 / Human / Abcam / ab80826 (1:100)

Histone H3 / D1H2 / Human / / Fluidigm / 3176023D (1:100)

Validation

All antibodies were individually titrated prior to use to identify optimal concentration and avoid non-specific staining. Staining specificity was controlled based on staining of cells from knock-out mice or by simultaneous analysis of known cell populations as follows:

IF/WB: Antibodies for western blot were validated based on specific binding at the expected molecular weight.

Immunofluorescence was validated with secondary-only staining and co-staining for expected overlapping markers. Neutrophil markers co-localized with Ly6G (human) or MPO (human), and vascular markers (including lectin) overlapped with CD31.

FACS/Flow cytometry: All flow antibodies were individually titrated prior to use. All experiments included single-stain and fluorescence-minus-one (FMO) controls for spectral deconvolution and gating.

In vivo neutrophil depletion: Antibody-based depletion in vivo was confirmed by flow cytometry using non-competing clones. The RB6-8C5 anti-Gr1 clone inhibits binding of Ly6G 1A8, therefore Ly6C/SSC was used to validate neutrophil depletion (ref. 10). Ly6C/SSC was also used to confirm anti-Ly6G/IgG2a specificity (ref. 18 and Supplementary Fig. 3d)

IMC: Antibody specificity for imaging mass cytometry was confirmed by simultaneous analysis of cell populations with known phenotypes. Leukocyte markers co-localized with CD45, myeloid markers with CD68 or CD14, lymphocyte markers with CD3 or 
CD20, and all others (aSMA, CD31, Vimentin, Collagen 1) were CD45-negative. Abcam antibodies used for IMC were specifically chosen because they are recombinant rabbit monoclonal yielding high specificity and knock-out validation performed by the vendor. All IMC antibodies were tested on multiple control tissues including spleen, tonsil, placenta, and tumor tissue, and validated by a clinical pathologist.

\section{Eukaryotic cell lines}

Policy information about cell lines

Cell line source(s)

Source of all cell lines can be found in the methods section: Primary HUVEC (Lonza), HMEC (ATCC), Py230 (ATCC), E0771 (CH3 Biosystems), MDA-MB-231 (ATCC). For assays involving mouse immune cell culture, primary cells were isolated from peripheral sources (i.e. blood or bone marrow) or from lung (mechanical dissociation), and enriched by FACS or MACS columns prior to use in functional assays.

Authentication

All non-primary cell lines were obtained from commercial sources therefore authentication to rule out inter- and intraspecies contamination was performed by the vendor. This included (1) PCR detection of species-specific variants of the cytochrome Coxidase I gene (COI analysis) to rule out inter-species contamination, (2) short tandem repeat (STR) profiling to rule out intra-species contamination, and (3) pathogen testing.

Mycoplasma contamination

All cell lines were confirmed negative for mycoplasma contamination via PCR.

Commonly misidentified lines (See ICLAC register)

No cell lines used in this study were found in the database of commonly misidentified cell lines that is maintained by ICLAC and NCBI Biosample.

\section{Animals and other organisms}

Policy information about studies involving animals; ARRIVE guidelines recommended for reporting animal research

Laboratory animals

Species, background strain, sex and age are listed below for each mouse model used in this study, and reported in the Methods section for each experiment.

- Diet-induced obesity (DIO) model: Mouse, C57BL6, female, purchased from Jackson Laboratory at 4 weeks of age, enrolled on low fat or high fat diet at 5 weeks of age for 15 weeks, experiments initiated at 20 weeks of age (maintained on diets throughout duration).

- Genetic-induced obesity (GIO) model: Mouse, C57BL6, female, purchased from Jackson Laboratory at 4 weeks of age, maintained on standard rodent diet, experiments initiated at 10 weeks of age.

- Spontaneous MMTV-PyMT model: Mouse, C57BL6, female, purchased from Jackson Laboratory at 4 weeks of age, enrolled on low fat or high fat diet at 5 weeks of age, tumor progression monitored until max 25 weeks of age (maintained on diets throughout duration).

- Nos2-/- mice: Mouse, C57BL6, female, purchased from Jackson Laboratory at 4 weeks of age, enrolled on high fat at 5 weeks of age or maintained on standard rodent diet for 15 weeks, experiments initiated at 20 weeks of age (maintained on diets throughout duration).

Wild animals

No wild animals were used in this study

Field-collected samples

No field-collected samples were used in this study.

Ethics oversight

All mouse procedures were reviewed and approved by McGill University Animal Care committee and conformed to the standards of the Canadian Council on Animal Care.

Note that full information on the approval of the study protocol must also be provided in the manuscript.

\section{Human research participants}

Policy information about studies involving human research participants

Population characteristics

We obtained biobanked lung metastasis tissue samples from male $(n=18)$ or female $(n=4)$ cancer patients, body mass index ranged from 19.9 - 36.3, age ranged from 46-86, primary cancer diagnoses included prostate, colon/colorectal, bladder, brain, breast, and esophageal.

Recruitment

All biobanked lung metastasis surgical specimens that had appropriate consent and body mass index information were included. Recruitment was not done specifically for our study. Due to the limited number of samples that were available, there is high representation male patients (18 out of 22 patients) and colon/colorectal cancer patients (15 out of 22 patients), therefore our findings may be bias towards these groups.

Ethics oversight

The protocol for human sample biobanking was approved by the Montreal University Health Centre Research Ethics Board (REB) on Feb 27, 2019, Protocol Number: 2019-5354.

Note that full information on the approval of the study protocol must also be provided in the manuscript. 


\section{Plots}

Confirm that:

Х The axis labels state the marker and fluorochrome used (e.g. CD4-FITC).

Х The axis scales are clearly visible. Include numbers along axes only for bottom left plot of group (a 'group' is an analysis of identical markers).

$\bigotimes$ All plots are contour plots with outliers or pseudocolor plots.

Х A numerical value for number of cells or percentage (with statistics) is provided.

\section{Methodology}

Sample preparation

Instrument

Software

Cell population abundance

Gating strategy

$\bigotimes$ Tick this box to confirm that a figure exemplifying the gating strategy is provided in the Supplementary Information.
Sample preparation for all flow cytometry or FACS experiments can be found in the Methods section. This includes FACS purification of neutrophils for RNA-seq and CellROX flow cytometry assays. Briefly, primary tissues were perfused with PBS prior to collection, mechanically dissociated, and filtered prior to staining. Whole blood was collected into tubes containing anticoagulant, and red blood cells were lysed (Pharm Lyse, BD Biosciences). Fc block (1 h, BD Biosciences); conjugated antibodies (1 h, see Supplementary Table 1); fixable live/dead stain (30 min, Invitrogen) or DAPI (10 min).

A BD LSRFortessa ${ }^{\mathrm{TM}}$ was used for flow cytometry, and a BD FACSAria III ${ }^{\mathrm{TM}}$ was used for fluorescence activated cell sorting (FACS)

FlowJo was used for all flow cytometry data analysis.

In all cases, post-sort purity was confirmed to be greater than $>90 \%$ for downstream applications.

Dead cells and debris were excluded from analyses using FSC x SSC, a live/dead stain and/or DAPI. CD45+ was used as a marker for total leukocytes, CD11b+ was used as a marker for myeloid cells. Neutrophils were further defined as CD45+CD11b+ Ly6CloLy6G+, monocytes as CD45+CD11b+Ly6ChiLy6G- and macrophages as CD45+CD11b+Ly6C-Ly6G-. 